US Army Corps of Engineers ${ }_{\circledast}$

Engineer Research and

Development Center

\title{
Laboratory Study to Reduce the Transport of Asian Carp by Barges
}

Duncan B. Bryant, Christopher Callegan, Lauren Coe, Jarrell Smith,

August 2018

Richard Styles, and Christine Moore

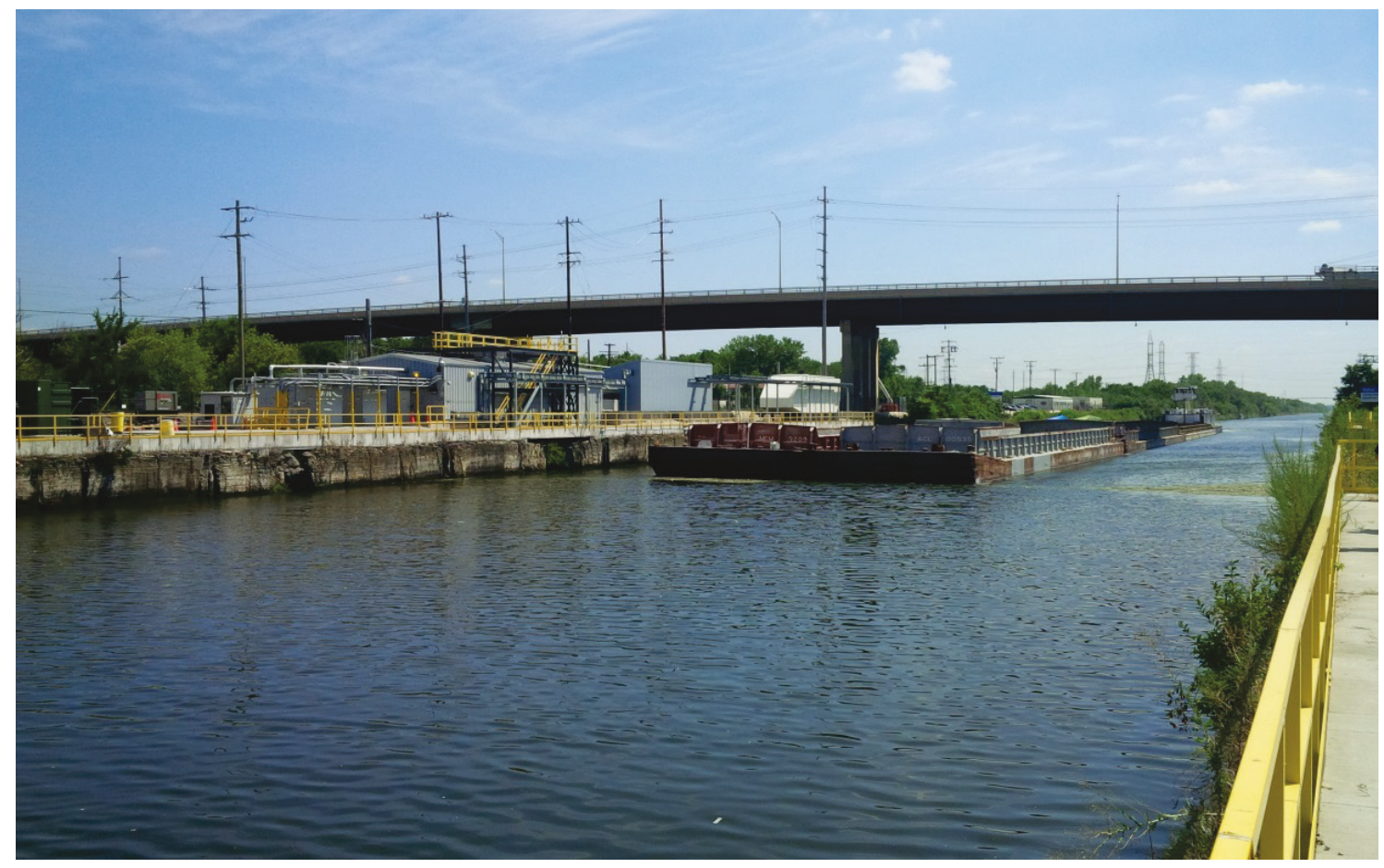

Approved for public release; distribution is unlimited. 
The U.S. Army Engineer Research and Development Center (ERDC) solves the nation's toughest engineering and environmental challenges. ERDC develops innovative solutions in civil and military engineering, geospatial sciences, water resources, and environmental sciences for the Army, the Department of Defense, civilian agencies, and our nation's public good. Find out more at www.erdc.usace.army.mil.

To search for other technical reports published by ERDC, visit the ERDC online library at http://acwc.sdp.sirsi.net/client/default. 


\section{Laboratory Study to Reduce the Transport of Asian Carp by Barges}

Duncan B. Bryant, Christopher Callegan, Lauren Coe, Jarrell Smith, Richard Styles, and Christine Moore

Coastal and Hydraulics Laboratory

U.S. Army Engineer Research and Development Center 3909 Halls Ferry Road

Vicksburg, MS 39180-6199

Final report

Approved for public release; distribution is unlimited.

Prepared for U.S. Army Engineer District, Chicago

111 North Canal Street, Suite 600

Chicago, IL 60606-7206

Under Project 114532, "CSSC Dispersal Barriers" 


\section{Abstract}

Potential migration of Asian carp through the Illinois River, Des Plaines River, and Chicago Area Waterway System is a risk facing the Great Lakes. The U.S. Army Corps of Engineers (USACE) has installed electric barriers within the Chicago Sanitary and Ship Canal (CSSC) near Romeoville, IL, to deter fish from using the canal as a pathway to enter the Great Lakes. Commercial tows operating within the CSSC produce residual currents that can entrain and potentially transport fish across the barrier. The U.S. Army Engineer Research and Development Center was tasked by the USACE Chicago District to investigate mitigation measures to remove entrained fish from the recesses between barges and to reduce effects of return currents in transporting fish across the barriers. A 1:16.7 scale physical model with remote-controlled tow and barges was used to evaluate mitigation strategies. For southbound tows, mitigation measures include maintaining a minimum discharge in the canal to overcome the return current or reducing vessel speeds across the barriers. For northbound tows, mitigation measures include vessel maneuvers and upward facing jets to dislodge or remove fish during passage. These jets were effective at flushing fish for lower vessel speeds (2.5 miles per hour).

DISCLAIMER: The contents of this report are not to be used for advertising, publication, or promotional purposes. Citation of trade names does not constitute an official endorsement or approval of the use of such commercial products. All product names and trademarks cited are the property of their respective owners. The findings of this report are not to be construed as an official Department of the Army position unless so designated by other authorized documents. 


\section{Contents}

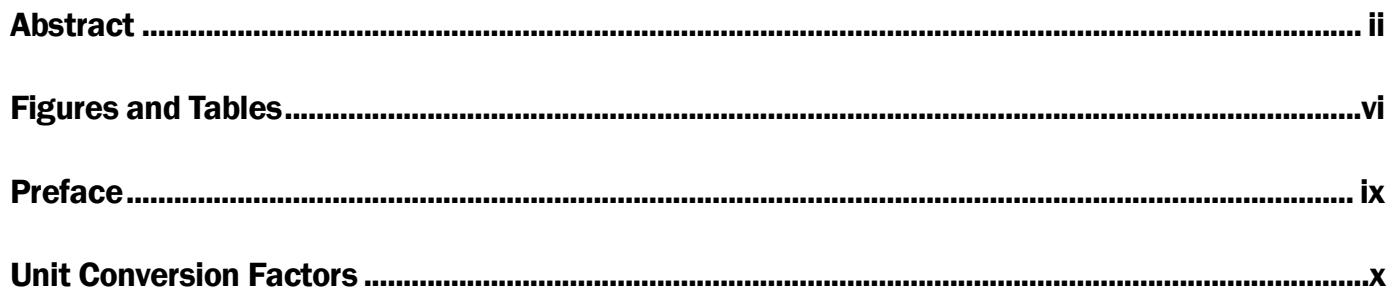

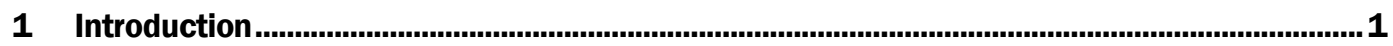

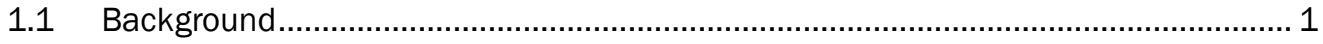

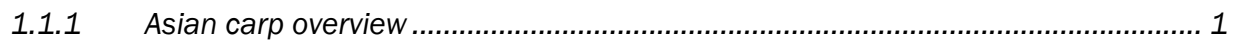

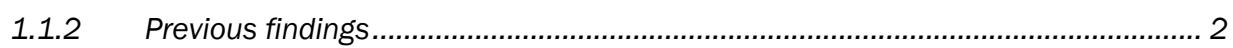

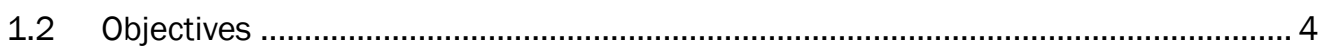

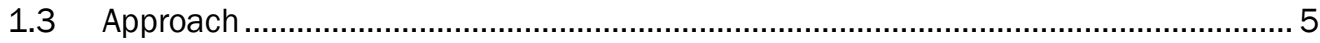

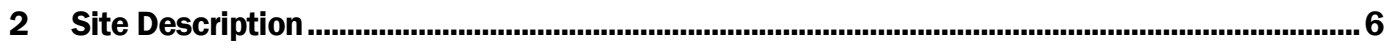

2.1 The Chicago Sanitary and Ship Canal (CSSC) ............................................... 6

2.2 Electric barriers in the CSSC ............................................................................ 11

2.3 Water motions near tows in confined channels .................................................. 12

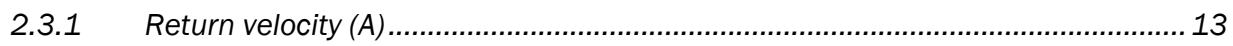

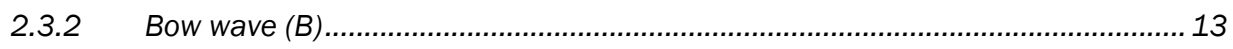

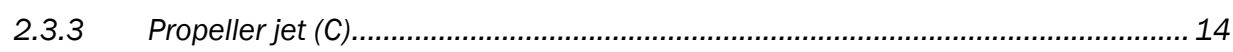

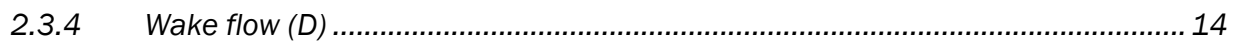

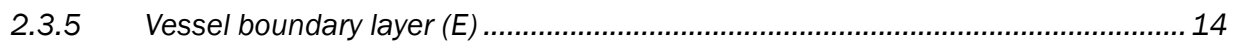

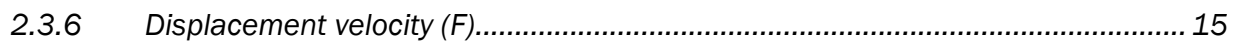

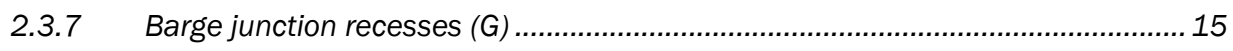

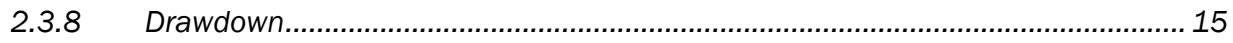

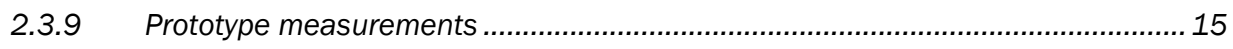

3 Methods ................................................................................................................................17

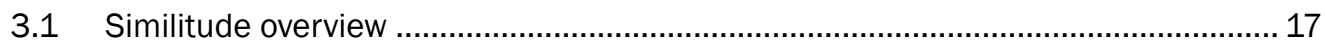

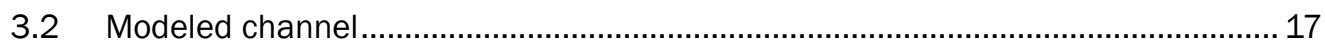

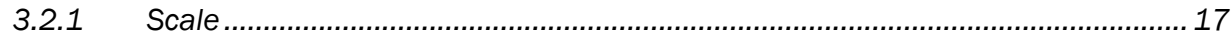

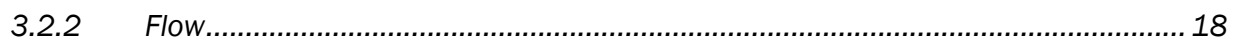

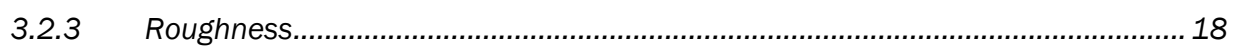

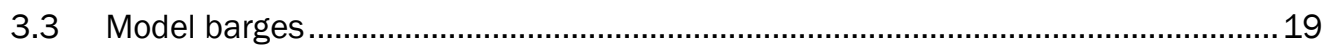

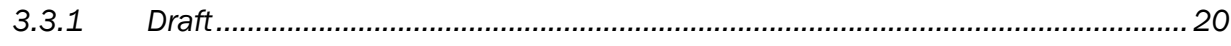

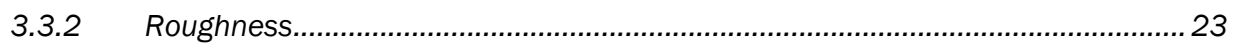

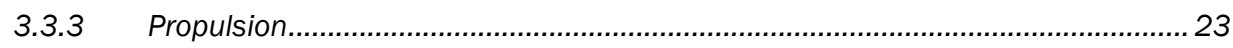

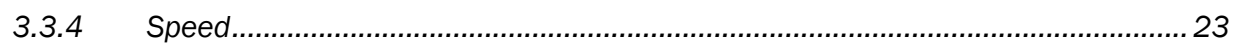

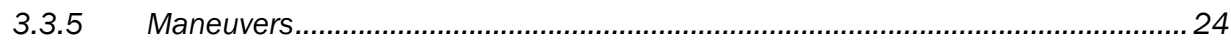

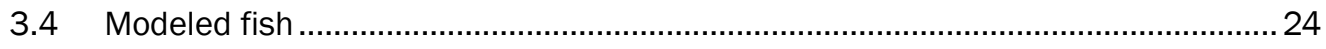

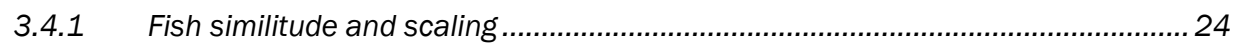

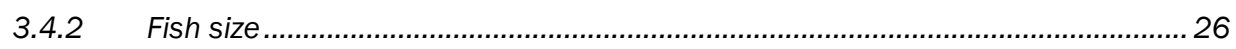

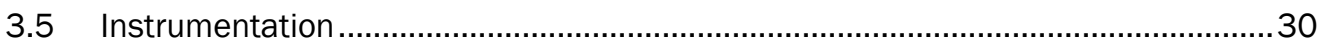


3.5.1 Acoustic Doppler Velocimetry (ADV)............................................................ 30

3.5.2 Ultrasonic flow meters ...................................................................................... 30

3.5.3 Electromagnetic current meter .......................................................................... 30

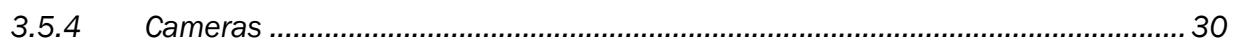

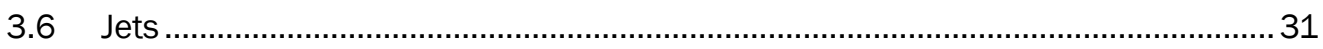

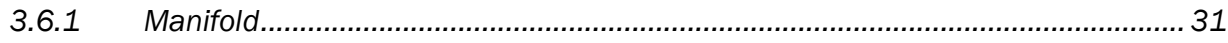

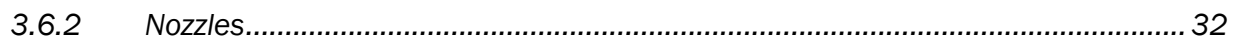

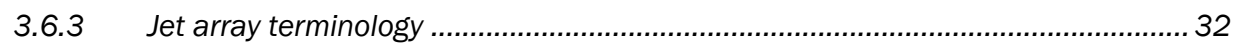

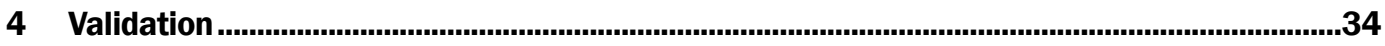

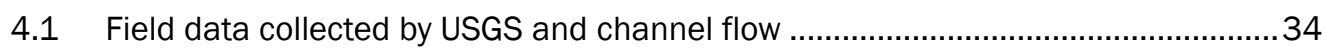

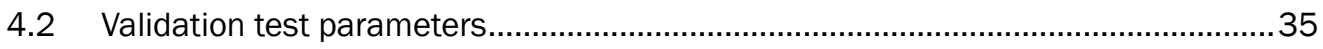

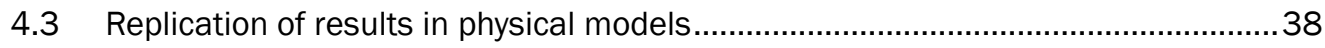

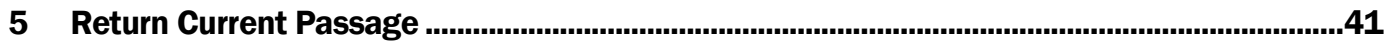

5.1 Specific methods for return currents .......................................................... 41

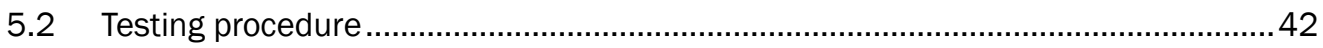

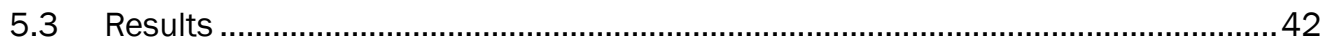

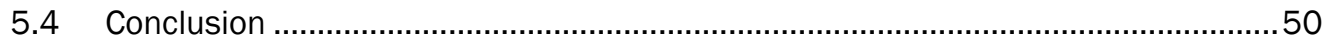

6 Entrainment and Flushing of Barge Junction Recesses ........................................................52

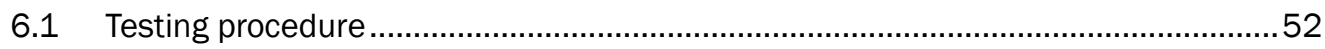

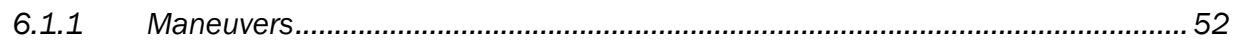

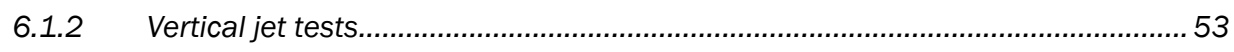

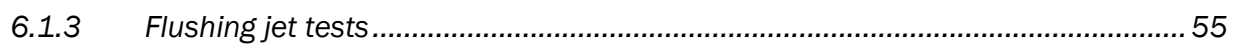

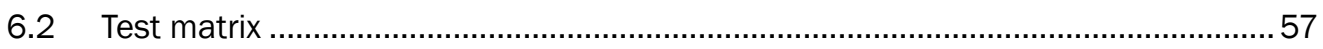

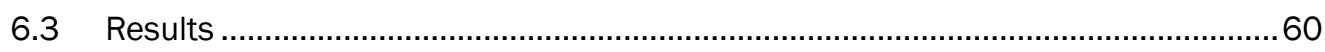

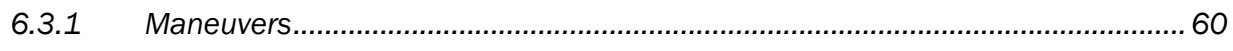

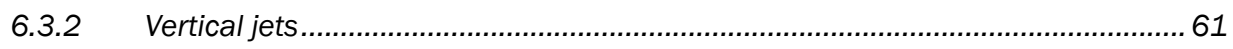

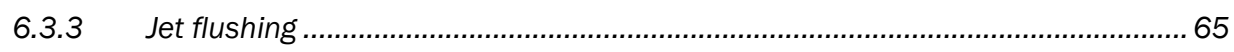

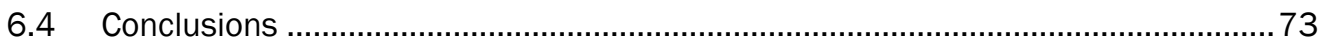

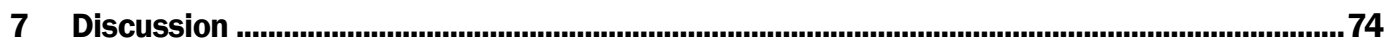

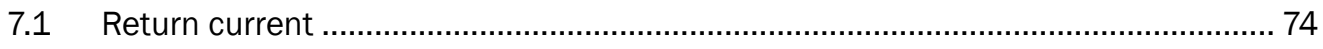

7.1.1 Spatial distribution of the return current............................................................... 74

7.1.2 Effect of the tow configuration ......................................................................... 75

7.1.3 Measures to mitigate the return current ........................................................... 76

7.1.4 Modeling return current at the barriers................................................................ 77

Entrainment - vessel maneuvers....................................................................... 77

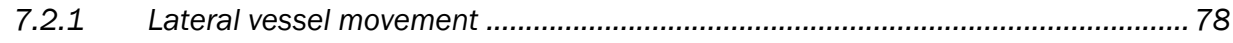

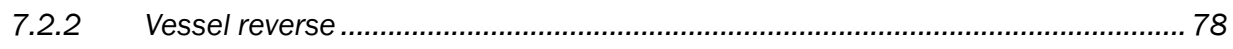

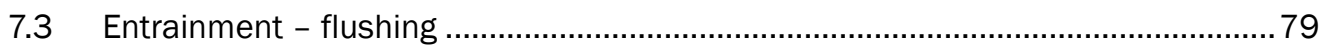

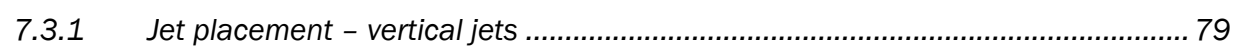

7.3.2 Jet placement - forward-angled jets, low vessel speed ....................................... 79

7.3.3 Jet placement - forward-angled jets, high vessel speed...................................... 81

7.3.4 Jet placement - canal flow............................................................................. 81

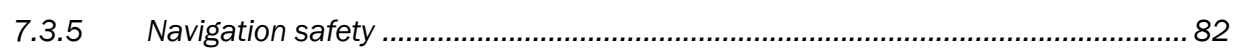


7.3.6 Jet manifold design alternatives that demonstrated lower levels

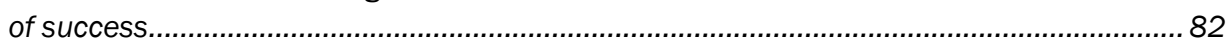

7.4 Other potential alternatives .............................................................................. 83

7.4.1 Return current blockage system ..................................................................... 84

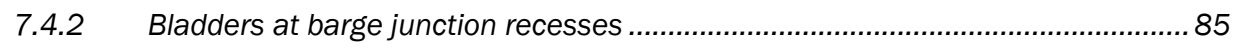

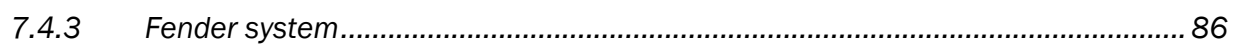

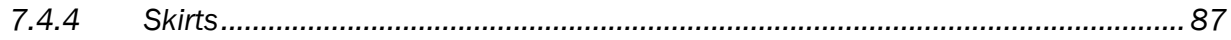

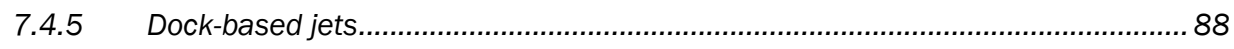

8 Summary and Conclusions .................................................................................................89

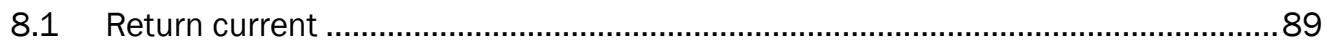

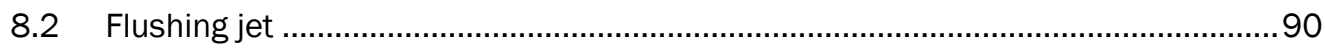

8.3 Other considerations and recommendations .....................................................90

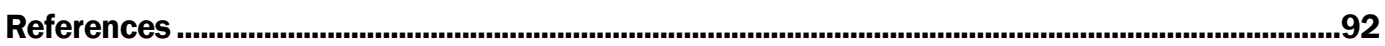

\section{Report Documentation Page}




\section{Figures and Tables}

\section{Figures}

Figure 1-1. Aerial photograph depicting the location of the electric barriers within the CSSC.

Figure 1-2. Summary of results from previous barge entrainment study. The vertical axis denotes the percentage of fish carried past the electric barrier (after Bryant et al. 2016)

Figure 2-1. Estimated discharge from USGS site 05536890 on the CSSC near Lemont, IL. Note: At the time of publication, these data were indicated as "provisional" by the USGS and are subject to revision

Figure 2-2. Estimated average cross-section velocity from USGS site 05536890 on the CSSC near Lemont, IL. Note: At the time of publication, these data were indicated as provisional by the USGS and are subject to revision.

Figure 2-3. Seasonal and daily tug passages past the electric barrier. (A) Daily passages by time of year. The filtered signal represents the 7-day moving average. "North" and "south" represent the direction of barge travel. (B) Passages by time of day. The filtered signal represents a 3-hour moving average.

Figure 2-4. Distributions of tug speed passing the barrier. (A) Northbound tugs,

(B) Southbound tugs.

Figure 2-5. Electric barriers on the CSSC.

Figure 2-6. Modes of water motions around tows moving left to right in confined channels. $A=$ return velocity; $B=$ bow wave; $C=$ propeller jet; $D=$ wake flow; $E=$ flow in boundary layer along hull; $\mathrm{F}=$ displacement flow at bow between hull and channel bottom having short duration; and $\mathrm{G}=$ pocket recirculation at barge junction recesses.

Figure 2-7. Near-bed velocities measured in $23 \mathrm{ft}$ water depth beneath barges and towboat (after Maynord 2000).

Figure 3-1. Barriers $2 \mathrm{~A}$ and $2 \mathrm{~B}$ in model test facility at $\mathrm{CHL}$. Wide-array mesh in foreground denotes parasitic structure; linear, narrow arrays in background denote the electrodes.

Figure 3-2. Asian carp test facility for navigation effects.

Figure 3-3. Schematic of boundary layer on hull and channel bottom for both model and full-scale tows.

Figure 3-4. Comparison of prototype fish Stokes number to model fish Stokes number for a fish-size eddy..

Figure 3-5. Comparison of prototype fish Stokes number to model fish Stokes number for a barge draft size eddy.

Figure 3-6. Comparison of prototype fish Stokes number to model fish Stokes number for a barge width-size eddy.

Figure 3-7. Comparison of three model fish with lengths of 1.5 in., 1.875 in., and 2.25 in.

Figure 3-8. Single manifold placed in the center of the channel parallel to the channel walls.

Figure 3-9. Manifold with 8 in. (prototype) nozzles. Nozzles are oriented at 45 deg off of the vertical and are rotated in an alternating pattern to force fluid obliquely to the path of the tow as it passes overhead.

Figure 3-10. Nozzle and jet variables for jet and manifold configurations 
Figure 4-1. The spatially averaged velocity for the three inner and three outer ADCP bins from $12: 54$ to $14: 05$ CDT.

Figure 4-2. Barge configuration the Lisa $E$. was pushing during the downstream return current testing on 31 August 2015.

Figure 4-3. Path of Lisa E. on 31 August 2015 from 12:48 to 13:10 CDT taken from AIS data. Red pin shows the location of the USGS ADCP measurements.

Figure 4-4. The over-ground speed of the Lisa E. between 12:47 and 13:10 CDT, taken from the AIS data. Red line indicates the tow passage by the USGS ADCP.

Figure 4-5. Picture of the channel setup with the three ADVs placed along the left wall.

The direction of flow is also shown.

Figure 4-6. Velocity comparison between model and prototype for the validation testing with a tow velocity of $3.39 \mathrm{mph}$.

Figure 4-7. Velocity comparison between model and prototype for the validation testing with a tow velocity of $3.41 \mathrm{mph}$..

Figure 5-1. Depths of return current velocity measurements......................................................... 41

Figure 5-2. Observed time of return current at six lateral positions and two depths with error bars showing standard error of the mean for a 2-wide by 3-long tow.

Figure 5-3. Mean of return current (feet/second) at six lateral positions and two depths with error bars showing standard error of the mean for a 2-wide by 3-long tow.

Figure 5-4. Calculated average displacement distances (feet) at six lateral positions and two depths with error bars showing standard error of the mean for a 2-wide by 3-long tow.

Figure 5-5. Mean observed time (minute) of return current for six barge configurations and two vessel speeds with calculated standard error.

Figure 5-6. Mean velcoity (ft/s) of return current for six barge configurations and two vessel speeds with calculated standard error.

Figure 5-7. Mean calculated return current displacement distances with the 98\% confidence interval.

Figure 5-8. Maximum measured return current displacements (feet) with the $99 \%$ onetailed confidence interval maximum displacement distances.

Figure 5-9. Channel flow effect on return current for a vessel over water speed of 1.71 $\mathrm{mph}$ at 4.1, 9.0, and $13.9 \mathrm{ft}$ from the canal wall.

Figure 6-1. Sample data sheet for maneuver flushing tests.

Figure 6-2. Location of ADVs, represented by $\mathbf{x}$, with respect to the jet array. The distance between adjacent instruments is 15 in.

Figure 6-3. Sample data sheet for jet flushing tests.

Figure 6-4. Model barge configurations used for flushing tests.

Figure 6-5. Average horizontal velocities $(\mathrm{u})$ for the first jet configuration with varying depths and flow rates at ADV Locations 1,2 , and 3.

Figure 6-6. Average horizontal velocities for the second jet configuration with varying depths and flow rates at ADV Locations 1, 2, and 3.

Figure 6-7. Average horizontal velocities for the second jet configuration with varying depths and flow rates at ADV Locations 4, 5, and 6.

Figure 6-8. Average longitudinal velocities for the second jet configuration with instruments at a $4 \mathrm{ft}$ depth and at ADV Locations 7, 8, and 9.

Figure 6-9. Average longitudinal velocities for the second jet configuration with instruments at a $4 \mathrm{ft}$ depth and at ADV Locations 1, 4, 10. 
Figure 6-10. Minimum flushing effectiveness for medium fish at $4 \mathrm{mph}$ and $5 \mathrm{mph} . . . \ldots \ldots \ldots \ldots \ldots . . . . . . . .68$

Figure 6-11. Minimum flushing effectiveness vs. channel flow rate................................................69

Figure 6-12. Minimum flushing effectiveness vs. model fish size. ................................................... 71

Figure 6-13. Proportion flushed for all test tests vs. over-ground speed.............................................72

Figure 6-14. Minimum flushing effectiveness vs. over-ground speed. ...............................................73

Figure 7-1. Manifold configuration for successful jet tests. Barge direction is from the top

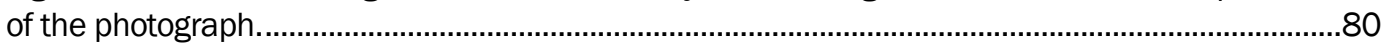

Figure 7-2. Schematic illustrating return current gate blocking system. Water flows through the mesh gates but prevents fish from moving with the return current. .

Figure 7-3. Schematic illustrating bumper system. Similar in concept to the gate in that fish are deflected downward into the high-voltage region.

Figure 7-4. Schematic illustrating possible alternatives for reducing fish entrainment.

(a) fender, (b) bladder and/or skirt.

Figure 7-5. Schematic illustrating docking maneuvers and jet wash to remove entrained fish.

\section{Tables}

Table 3-1. Scaling of parameters from model to prototype scale.................................................17

Table 3-2. Displacement thickness calculations. Model scale $=1: 16.66$. Tow speed $=4 \mathrm{mph} . \ldots \ldots \ldots . . . .22$

Table 6-1. Testing matrix for maneuver flushing tests. ..................................................................57

Table 6-2. Testing matrix for jet flushing tests...................................................................................58

Table 6-3. Results for maneuver flushing tests...........................................................................61

Table 6-4. Results for Level 1 jet flushing tests...............................................................................65

Table 6-5. Results of Level 2 jet flushing tests.................................................................................67

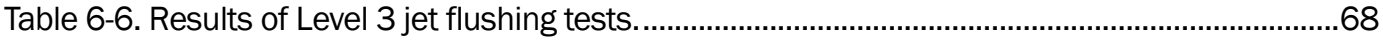

Table 6-7. Results of Level 4 jet flushing tests. .................................................................................69

Table 6-8. Results of Level 4 jet flushing tests with large and small model fish...............................70

Table 6-9. Results of Level 4 alternate barge configurations for jet flushing tests............................ 71 


\section{Preface}

The investigation reported herein was sponsored by the U.S. Army Engineer District, Chicago, under Project 114532, "CSSC Dispersal Barriers." This work was conducted at the Coastal and Hydraulics Laboratory (CHL) of the U.S. Army Engineer Research and Development Center (ERDC) from November 2015 through August 2016.

This research was conducted under the general direction of Mr. José E. Sánchez, Director, CHL; Dr. Kevin Barry, Deputy Director, CHL; Dr. Jackie S. Pettway, Chief, Navigation Division, CHL; Dr. Ty Wamsley, Chief, Flood and Coastal Protection Division, CHL; Mr. Mark Gravens, Chief, Coastal Processes Branch, CHL; Mr. Thad Pratt, Chief, Field Data Collection Branch, CHL; and Ms. Tanya Beck, Chief, Coastal Engineering Branch, CHL. Mr. W. Jeff Lillycrop was the ERDC Technical Director for Civil Works, and Navigation Research, Development, and Technology Transfer portfolio.

At the time of publication of this report, Mr. Jeffrey R. Eckstein was Acting Director of CHL, and Mr. John T. Tucker III was Acting Deputy Director.

COL Ivan P. Beckman was the Commander of ERDC, and Dr. David W. Pittman was the Director. 


\section{Unit Conversion Factors}

\begin{tabular}{|l|l|l|}
\hline Multiply & \multicolumn{1}{|c|}{ By } & To Obtain \\
\hline cubic feet & 0.02831685 & cubic meters \\
\hline degrees (angle) & 0.01745329 & radians \\
\hline feet & 0.3048 & meters \\
\hline inches & 0.0254 & meters \\
\hline miles (nautical) per hour & 1.0000 & knots \\
\hline miles (statute) & 0.8684 & miles (nautical) \\
\hline miles (statute) & 1.6093 & kilometers \\
\hline
\end{tabular}




\section{Introduction}

\subsection{Background}

\subsubsection{Asian carp overview}

Asian carp were originally imported into the southern United States in the 1970s to help maintain wastewater treatment facility retention ponds and to provide fresh fish for human consumption. The potential migration of Asian carp through the Illinois River, Des Plaines River, and Chicago Area Waterway System (CAWS) is a severe risk factor facing the Great Lakes. Of the four species of Asian carp, Bighead and Silver Carp pose the most ecological risk to U.S. waters. These fish are voracious eaters and compete directly with native planktivores, juvenile fishes, and mussels.

In an attempt to prevent Asian carp from entering the Great Lakes, the U.S. Army Corps of Engineers (USACE) has erected a dispersal barrier system on the Chicago Sanitary and Ship Canal (CSSC). The CSSC is a man-made canal between the Great Lakes and Mississippi River basin that was completed in the early twentieth century to improve water quality in southern Lake Michigan and to provide a navigable canal between the Great Lakes and Mississippi River. The electric barriers in this system create an electric current in the water that deters fish from swimming through the CSSC and into Lake Michigan. Longer fish are more easily deterred than shorter fish because a higher voltage difference develops across their greater length when passing through the barrier.

The Electric Dispersal Barriers are located near Romeoville, IL, in the CSSC within the CAWS (Figure 1-1). The barriers are made of steel electrodes that are secured to the bottom of the CSSC. The electrodes are connected to a raceway consisting of electric connections to a control building. Equipment in the control building generates a direct current pulse through the electrodes, creating an electric field in the water that serves as a barrier to certain fish. 
Figure 1-1. Aerial photograph depicting the location of the electric barriers within the CSSC.

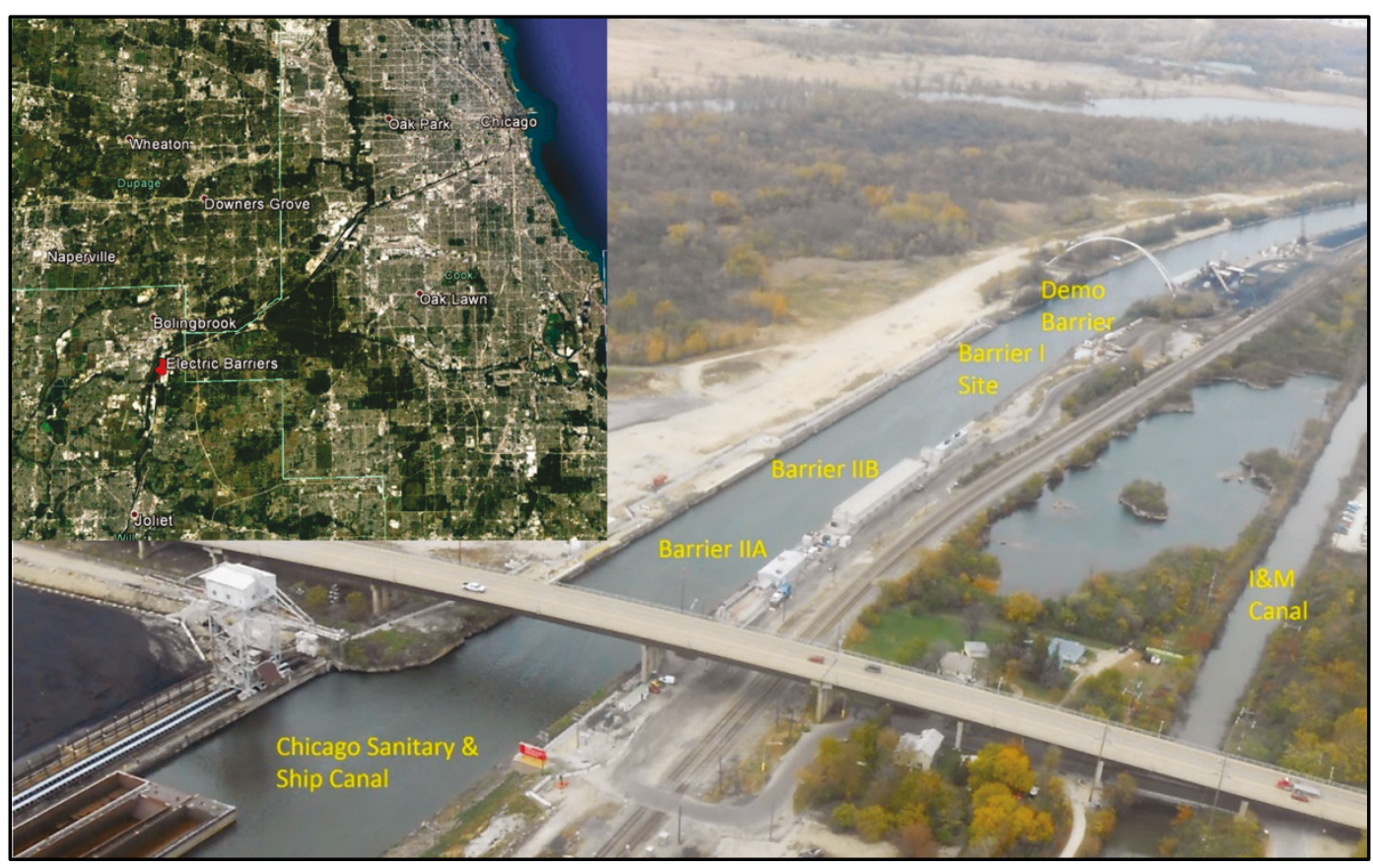

As of October 2014, Bighead and Silver Carp had not been observed at the electric barriers; however, a single Bighead Carp was captured in the Lockport pool downstream of the barriers in December 2009.

\subsubsection{Previous findings}

In 2011, the U.S. Army Engineer District, Chicago (LRC), requested the U.S. Army Engineer Research and Development Center (ERDC), Coastal and Hydraulics Laboratory (CHL), to determine if commercial navigation could cause or facilitate transport of Asian carp past the electric barriers at some time in the future (Bryant et al. 2016). It was hypothesized that currents generated by moving vessels or the vessel structure itself could inadvertently entrain fish, where they would be carried along with the vessel across the electric dispersal barriers. Once passed, the fish would be free to swim farther upstream and potentially into Lake Michigan. The commercial navigation vessels of interest are referred to as tows and consist of various numbers of barges that are lashed together and a pusher boat referred to as a towboat. ERDC recommended that a physical model of the CSSC and a model tow would enable evaluation of the potential for tows to transport Asian carp past the barrier.

The first objective of this prior study initiated in 2011 (Bryant et al. 2016) was to develop an understanding of the different mechanisms associated 
with tow passage that could lead to fish transport across the barrier. The second objective was to quantify the potential for movement of fish for each mechanism. The final objective was to discuss methods to minimize or eliminate the potential for transport across the barrier.

A 1:16.7 scale physical model with remote-controlled towboat and barges was used to evaluate the interactions of vessel, fluid motions, and nearly neutrally buoyant objects (model Asian carp) under a variety of vessel speeds and barge configurations typical of the CSSC near the electric barriers (Bryant et al. 2016). The primary tow configuration consisted of six barges in a 2-wide by 3-long flotilla. Other configurations including a 1-wide by 3 -long flotilla were investigated as well. Four of the barges had a rake, sloped leading edge, at one end, and the other two were boxed, vertical leading edge, at both ends. Multiple barge configurations including rakebox, rake-rake, and box-box connections were investigated. Three model fish sizes (lengths of 1.5 inch [in.], 1.875 in., and 2.25 in.) were used to represent stunned Asian carp. The fish were modified with closed-core foam and fine copper wire to achieve nearly neutral buoyancy conditions. Three tow speeds $(2,4$, and 6 miles per hour [mph]) were chosen to represent the typical range of commercial vessel speeds in the CSSC near the barriers.

To conform to the natural flow in the CSSC, the tests were divided into northbound (upstream) and southbound (downstream) directions, which placed the tows in different hydrodynamic regimes. Northbound tows traveled against the ambient current in the canal but toward Lake Michigan and produced the greatest concern for transporting entrained fish. Southbound tows traveled with the ambient flow of the canal and carried entrained fish away from Lake Michigan. The primary concern with southbound tows was the vessel-induced return current, which transported fish in the direction opposite the vessel direction (toward Lake Michigan).

For southbound traffic, the return velocity was found to be most relevant for model fish transport (Figure 1-2, A\&C). Narrower (1-wide) and slower tows produced less flow blockage and therefore showed lower potential for model fish movement. Further, tows with a leading raked barge produced less fish transport than a leading square barge. The results indicated that to reduce the risk of fish transport through the barrier, southbound tows should travel at minimal safe slow speed with a raked front barge. 
Figure 1-2. Summary of results from previous barge entrainment study. The vertical axis denotes the percentage of fish carried past the electric barrier (after Bryant et al. 2016).

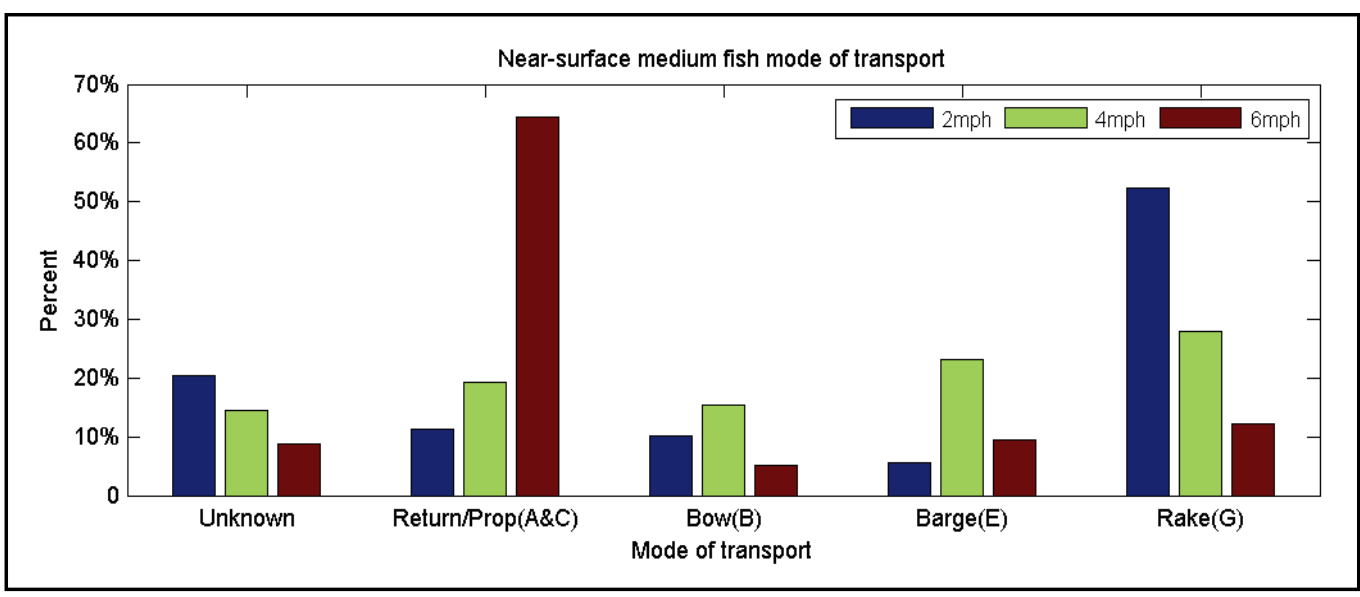

Northbound traffic produced transport by four different modes. The first mode examined was the bow wave, which was shown to transport model fish short distances. The second mode examined was transport within the barge boundary layer, which produced model fish transport through the electric barrier. The final two modes were entrainment in vortices that make up the wake flow and entrainment in the vortices that exist within voids in the barge configurations. These two modes transported fish the greatest distances, sometimes up to 2,000 feet ( $\mathrm{ft}$ ) beyond the end of the electric barrier.

Figure 1-2 provides a summary of test results from the prior study. The effect of different barge configurations, total distance traveled by fish, or time of transport is not directly addressed. Bryant et al. (2016) provide detailed information regarding Asian carp barge entrainment mechanisms, including individual test results.

\subsection{Objectives}

The Bryant et al. (2016) study initiated in 2011 demonstrated the degree to which commercial tow operations could transport fish across the electric barriers. As a follow-up to that study, LRC requested the ERDC CHL to investigate mitigation measures to remove or dislodge entrained Asian carp and determine the efficacy of such measures.

The first goal of this present study was to develop and test methods to dislodge or remove fish from the recesses between barges and from vesselinduced eddies and currents using submerged water jets before vessels 
cross the barriers. The second goal was to develop and evaluate alternatives to reduce the effect of the return current using a combination of vessel maneuvers and varying channel discharges. The alternatives tested in this study were chosen to lessen the need to delay or obstruct navigation. As such, alternatives that require vessel modifications were not considered. However, the final goal of this study was to review other alternatives that require vessel or barge modifications that were not tested but could be used to prevent fish transport across the barriers.

\subsection{Approach}

At the request of the LRC, the ERDC was tasked to investigate mitigation measures to remove entrained fish from the recesses between barges and to reduce the effect of the return current in transporting fish across the barriers. A series of tests were carried out in a 1:16.7 scale physical model that represented the CSSC near the electric barriers. The tests included measurements of the return current to determine particle displacement and installation of an array of upward-looking jets to displace entrained model fish from the recess between barge junction recesses.

The return current in the physical model was validated by measurements collected in the CSSC. The shape and magnitude of the return velocity was well correlated with the prototype. As such, the return current characteristics measured in the physical model were used to help explain the hydrodynamic conditions due to vessel passage at the electric barriers.

The flushing alternatives were designed to remove the fish before crossing the barrier and applied to northbound tows. The design included a manifold placed on the bottom of the canal with a series of upward-facing jets that impinged on the bottom of the barge during passage. A number of trial tests with different manifold and jet configurations were conducted to determine which method removed the greatest number of fish. 


\section{Site Description}

The electric barriers that are the focus of this study are located near Romeoville, IL, in the CSSC within the CAWS (Figure 1-1). This chapter provides site information about the canal, the electric barriers, vessel traffic in the canal, and the flow mechanisms around the tow and barges during navigation.

\subsection{The Chicago Sanitary and Ship Canal (CSSC)}

At the barrier, the CSSC is $160 \mathrm{ft}$ wide and has a rectangular cross section. Water depth varies from 20 to $28 \mathrm{ft}$, with $28 \mathrm{ft}$ being typical and $20 \mathrm{ft}$ only occurring when the canal is drained at Lockport Lock to prevent flooding in Chicago during heavy rainfall. The CSSC has a straight alignment for approximately 3.5 miles south and 0.3 mile north of the barrier. Flow in the channel is generally low. Discharge estimates between 2011 and 2015 (Figure 2-1) near Lemont, IL (U.S. Geological Survey [USGS] site 05536890, jointly operated by the USGS and the USACE) indicate that channel discharge is commonly between 1,500 and 3,500 cubic feet per second (cfs) and is greater than 4,500 cfs only approximately $10 \%$ of the time. In less than $1 \%$ of the record, lock and hydropower pump operations and/or wind can cause water to flow north toward Lake Michigan. However, these events are weak and short lived.

The flow estimates from Lemont were coupled with the typical crosssectional area of the CSSC at the barrier to generate estimates of average velocity at the barrier (Figure 2-2). The median cross-sectionally averaged velocity is $0.55 \mathrm{ft} /$ second (s), and the velocities exceeded at the 8oth, 9oth, and 95th percentiles are $0.88,1.10$, and $1.30 \mathrm{ft} / \mathrm{s}$, respectively. 
Figure 2-1. Estimated discharge from USGS site 05536890 on the CSSC near Lemont, IL. Note: At the time of publication, these data were indicated as "provisional" by the USGS and are subject to revision.

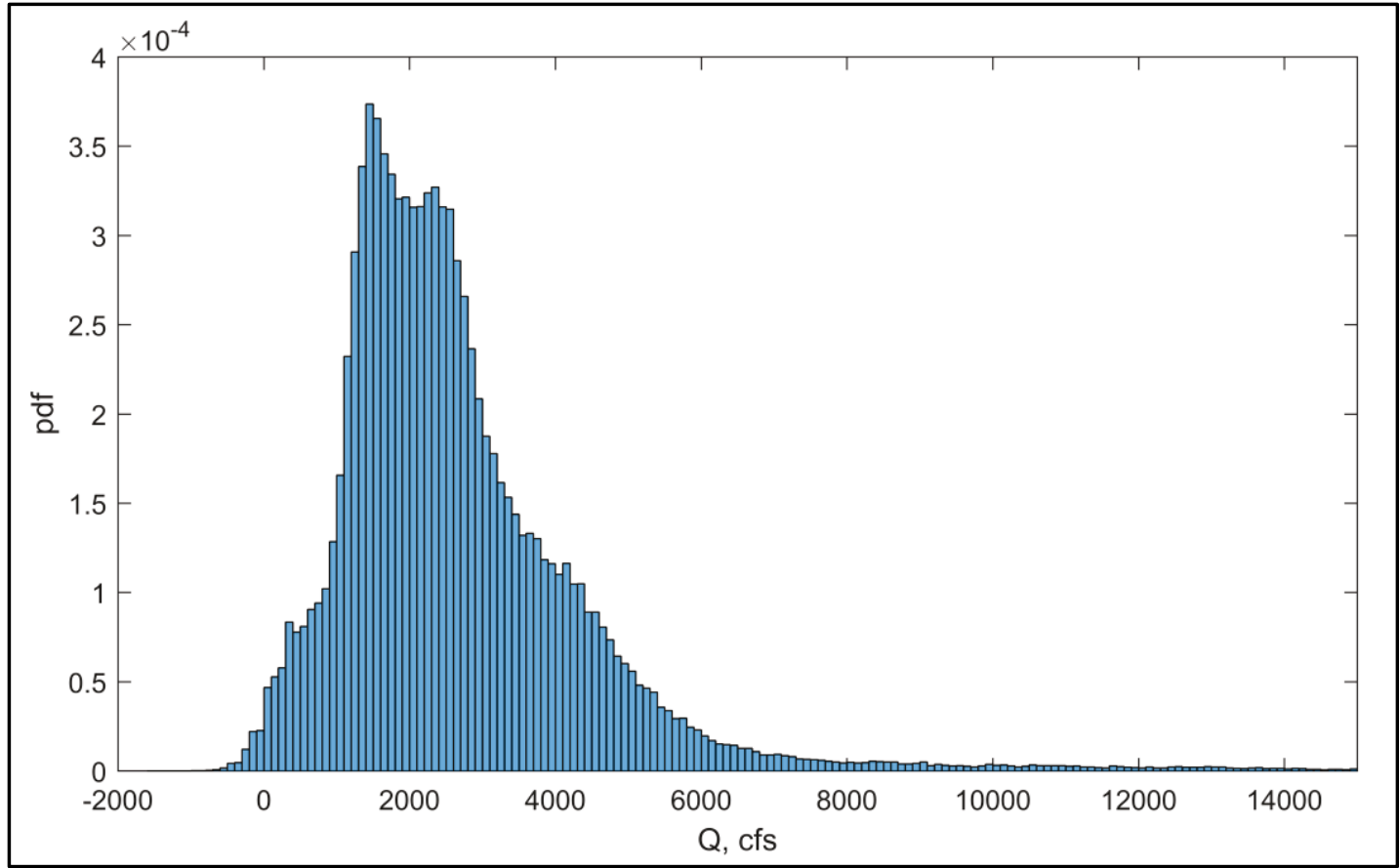

Figure 2-2. Estimated average cross-section velocity from USGS site 05536890 on the CSSC near Lemont, IL. Note: At the time of publication, these data were indicated as provisional by the USGS and are subject to revision.

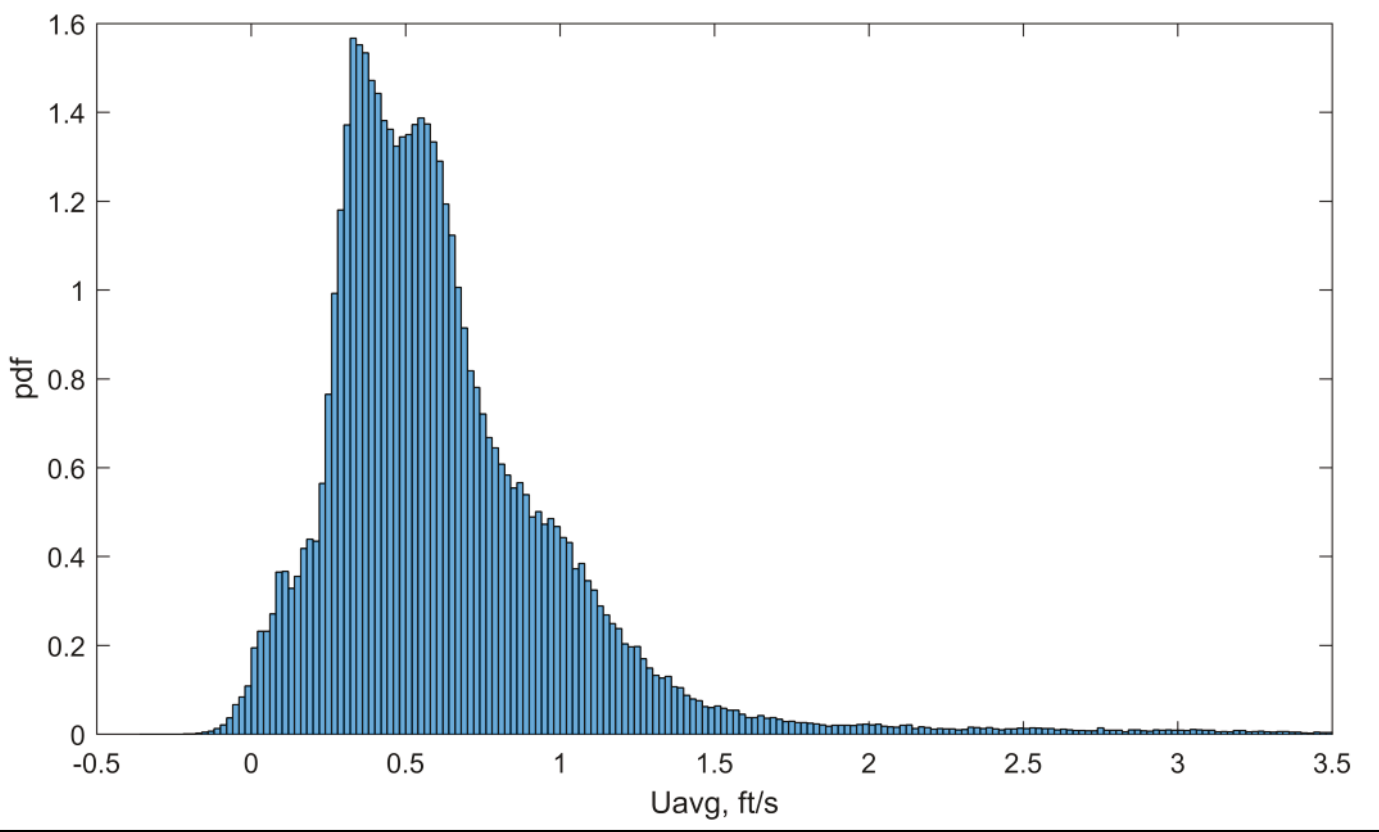


Commercial and recreational vessels use the CSSC. Commercial vessels, particularly those pushing loaded barges, are of particular interest for this study. To better understand the characteristics of these vessels, an analysis of vessel speed and barge conditions was conducted, use data from the Automatic Identification System (AIS) and the USACE Lock Performance Monitoring System (LPMS). USCG manages a database of AIS data, which provides positioning and various other identifying information and characteristics for vessels outfitted with an AIS transponder. The USACE maintains the LPMS database, which includes information regarding vessels and barge rafts that transit USACE locks. Records from these databases were retrieved for calendar years 2013 through 2015 to characterize the barge tows passing the electric barrier in the CSSC. Vessels transiting the CSSC from the electric barrier to Lockport Lock and Dam (L\&D) were extracted from the AIS database. For the same period, records for lockages at Lockport were obtained from the LPMS database.

The AIS database provided the vessel name and type for each vessel passing through the barrier-to-Lockport search box. To isolate commercial tow passages from other vessels (such as pleasure craft), vessel types matching tug, cargo, or tanker were selected. These vessel types represented $89 \%$ of vessel passages over the 3 -year record. On average, seven tugs pass the electric barrier each day. The number of passages per day (Figure 2-3, A) is relatively constant on the seasonal scale, with no statistically significant seasonal trends in total number of passages or direction of travel. Additionally, there appears to be no significant time-ofday bias for vessel passages (Figure 2-3, B). 
Figure 2-3. Seasonal and daily tug passages past the electric barrier. (A) Daily passages by time of year. The filtered signal represents the 7-day moving average. "North" and "south" represent the direction of barge travel. (B) Passages by time of day. The filtered signal represents a 3-hour moving average.

A)

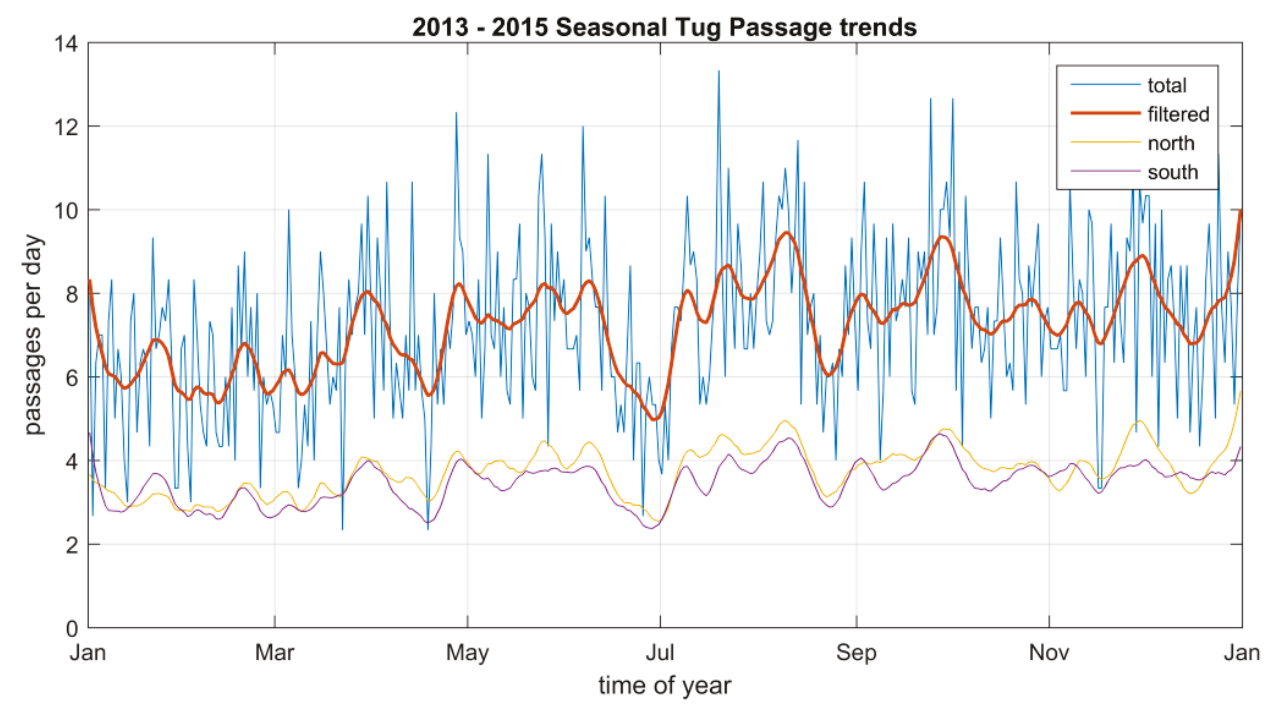

B)

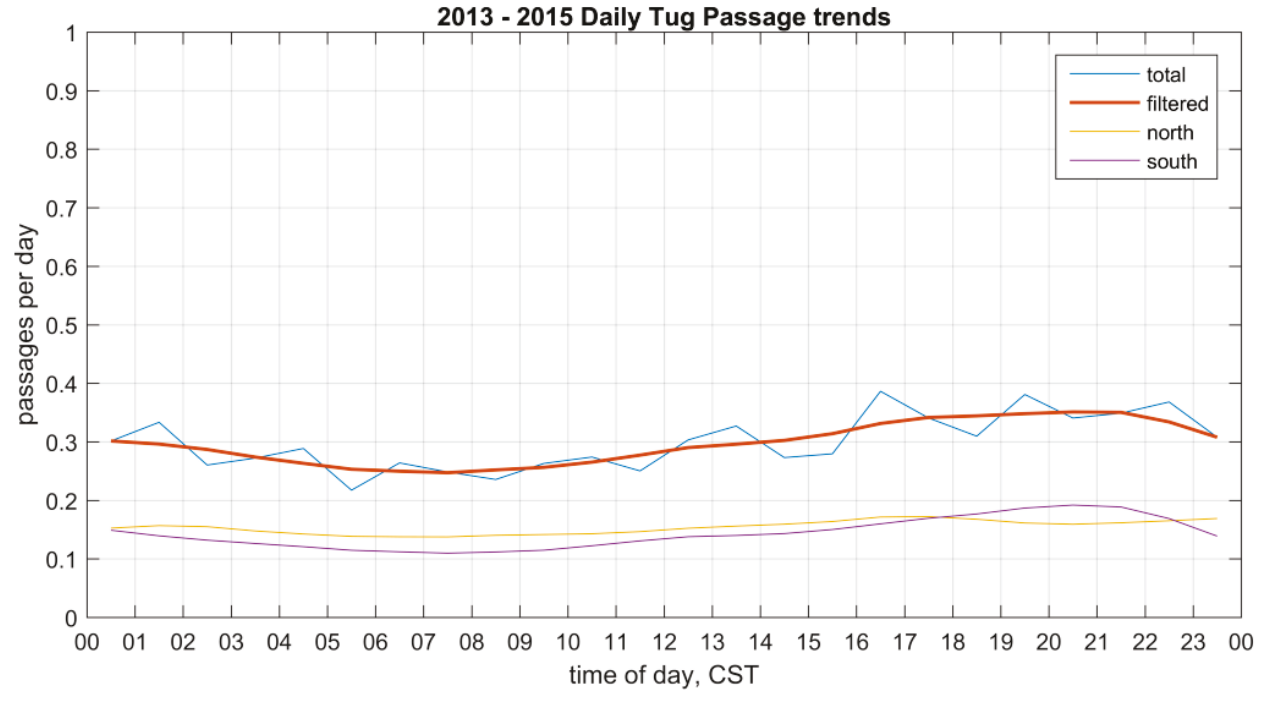

The AIS log gives instantaneous vessel speed with 1-knot precision (1 nautical $\mathrm{mph}$ ), which was considered insufficient resolution for the purposes of this study. A higher-precision velocity estimate was obtained by dividing the vessel track length while passing the barrier by the elapsed time of the passage. Additionally, vessel speed was estimated in two ways: speed over water and speed over ground. Speed over ground was estimated directly from the positioning track provided by AIS. Speed over 
water was estimated by determining the speed of the vessel relative to the average flow velocity in the channel (derived from the flow data from the Lemont gage).

The AIS database provides no information for barge configuration or load status, which are important factors for the return current generated (Bryant et al. 2016). The LPMS database was cross-referenced for each towboat passing the barrier to determine the number of loaded barges that the towboat was pushing. The LPMS data originate at Lockport L\&D, some 3.5 miles from the barrier, and barges often stop to reconfigure between Lockport and the barrier. Therefore, for the LPMS barge information to be associated with a passing towboat, the transit time between the barrier and the lock was required to be less than 3 hours. The resulting dataset is diminished because of excluded tows that reconfigured between the barrier and Lockport Lock and local barge traffic (those not passing through the lock). However, the dataset is sufficiently large, with 2,247 northbound tows and 2,428 southbound tows resolved, providing relationships between vessel speed, direction of travel, and the number of loaded barges the towboat was pushing (Figure 2-4).

Figure 2-4. Distributions of tug speed passing the barrier. (A) Northbound tugs, (B) Southbound tugs.

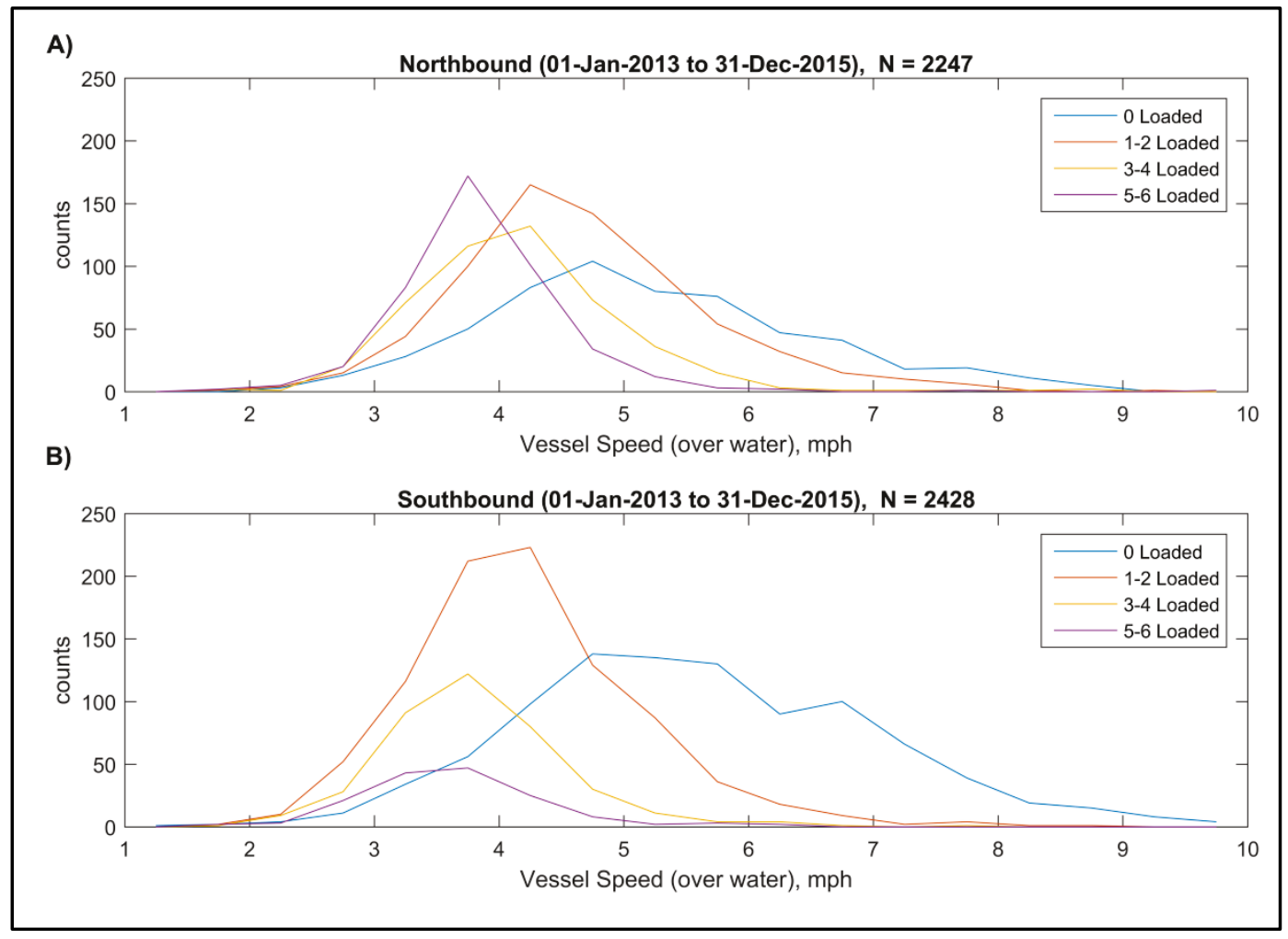


Vessel speed while passing through the barrier varies with the number of loaded barges. For both northbound and southbound traffic, the fastest towboat passages were associated with unloaded towboats. The peaks and maxima of the velocity distributions over water generally decreased as number of loaded barges increased. For the largest tows, with five to six loaded barges, although the vessel counts differed by direction, the normalized distributions were quite similar. The median, 90th, 95th, and 99th percentile speeds were $3.6,4.5,4.9$, and $5.9 \mathrm{mph}$ for southbound tows and $3.8,4.6,5.0$, and $6.1 \mathrm{mph}$ for northbound tows.

\subsection{Electric barriers in the CSSC}

The Electric Dispersal Barriers are located near Romeoville, IL, in the CSSC within the CAWS. The barriers are constructed of steel electrodes that are secured to the bottom of the CSSC. The electrodes are connected to a raceway consisting of electric connections to a control building, located adjacent to the canal. Equipment in the control building generates a direct current pulse through the electrodes, producing an electric field in the water that presents a barrier to certain fish.

There are three electric barriers: Demonstration Barrier, Barrier 2A, and Barrier 2B (Figure 2-5). The Demonstration Barrier has been operational since 2002. Due to its original demonstration status, it was designed and built with materials that were not intended for long-term use. Significant repairs were successfully completed in October 2008. In July 2013, USACE awarded a construction contract to build permanent electric Barrier 1 between Barrier 2B and the Demonstration Barrier. The Demonstration Barrier will be incorporated into the design of Permanent Barrier 1 and will be used as a behavioral barrier on the upstream side.

The Demonstration Barrier operates at 1 volt per in. (V/in.), at a frequency of 5 hertz (Hz), with 4-millisecond (ms) pulse duration. Barrier 2A was placed into full-time operation in 2009. Barrier $2 \mathrm{~B}$ was activated in April 2011 with the same operating parameters as Barrier 2A. Currently, Barrier 2A and 2B are operating at the following settings: $2.3 \mathrm{~V} /$ in., $34 \mathrm{~Hz}$, and $2.3 \mathrm{~ms}$ pulse duration. 
Figure 2-5. Electric barriers on the CSSC.

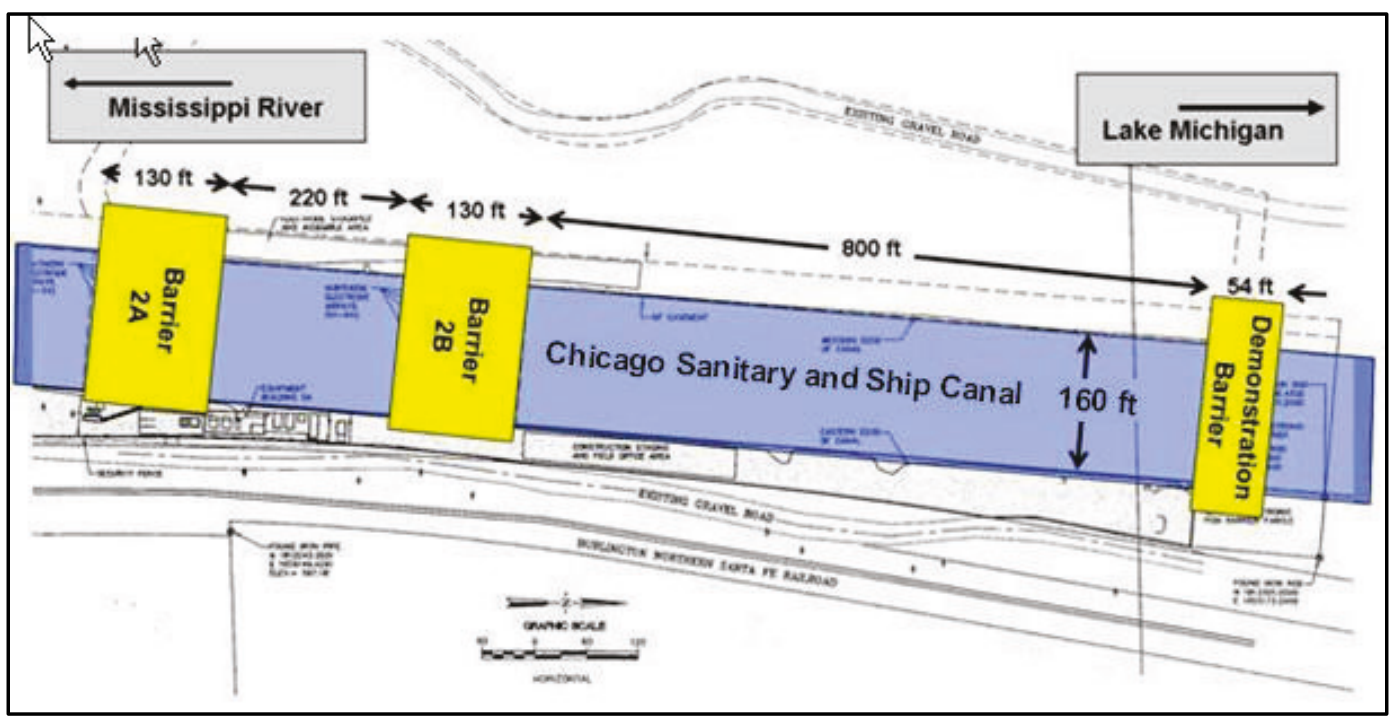

\subsection{Water motions near tows in confined channels}

A confined channel is one in which the vessel occupies a significant portion of the channel cross-sectional area. When a vessel occupies a large portion of the channel cross-sectional area, flows generated by vessel motion are restricted to a smaller area and become magnified. A measure of a confined channel is the blockage ratio $(\mathrm{N})$, which is the area ratio of vessel cross section to channel cross section. There is no well-established limit to describe when a channel becomes confined, but a blockage ratio of 0.05 will exhibit flow conditions considered to be typical of confined channels at sufficiently high vessel speed. Note that the CSSC at $25 \mathrm{ft}$ depth by $160 \mathrm{ft}$ width and a 2-wide loaded barge tow $(35 \mathrm{ft} \times 2=70 \mathrm{ft}$ wide and $9 \mathrm{ft}$ draft $)$ has a blockage ratio of $(9 \mathrm{ft} \times 70 \mathrm{ft}) /(25 \mathrm{ft} \times 160 \mathrm{ft})=0.16$ that is significantly confined.

Navigation in confined channels has been studied by numerous investigators. Many of the previous studies such as Schijf (1949) and PIANC (1987) have focused on channel stability and bank erosion effects. Studies on the upper Mississippi River (Maynord 2000) focused on the environmental effects of navigation. To the authors' knowledge, no previous literature exists for fish transport by vessels in a significantly confined waterway like the CSSC.

One of the most obvious water motions generated by tows is the propeller jet(s). Almost all towboats of the size found in the CSSC have two propellers, and velocities exiting these propellers can approach $30 \mathrm{ft} / \mathrm{s}$ at 
full throttle. In a confined channel, other water motions are present due to various factors including the displacement effects of the tow. Water motions generated by tows are illustrated in Figure 2-6 and described in the following paragraphs.

Figure 2-6. Modes of water motions around tows moving left to right in confined channels. $A=$ return velocity; $B=$ bow wave; $C=$ propeller jet; $D=$ wake flow; $E=$ flow in boundary layer along hull; $F=$ displacement flow at bow between hull and channel bottom having short duration; and $\mathrm{G}=$ pocket recirculation at barge junction recesses.

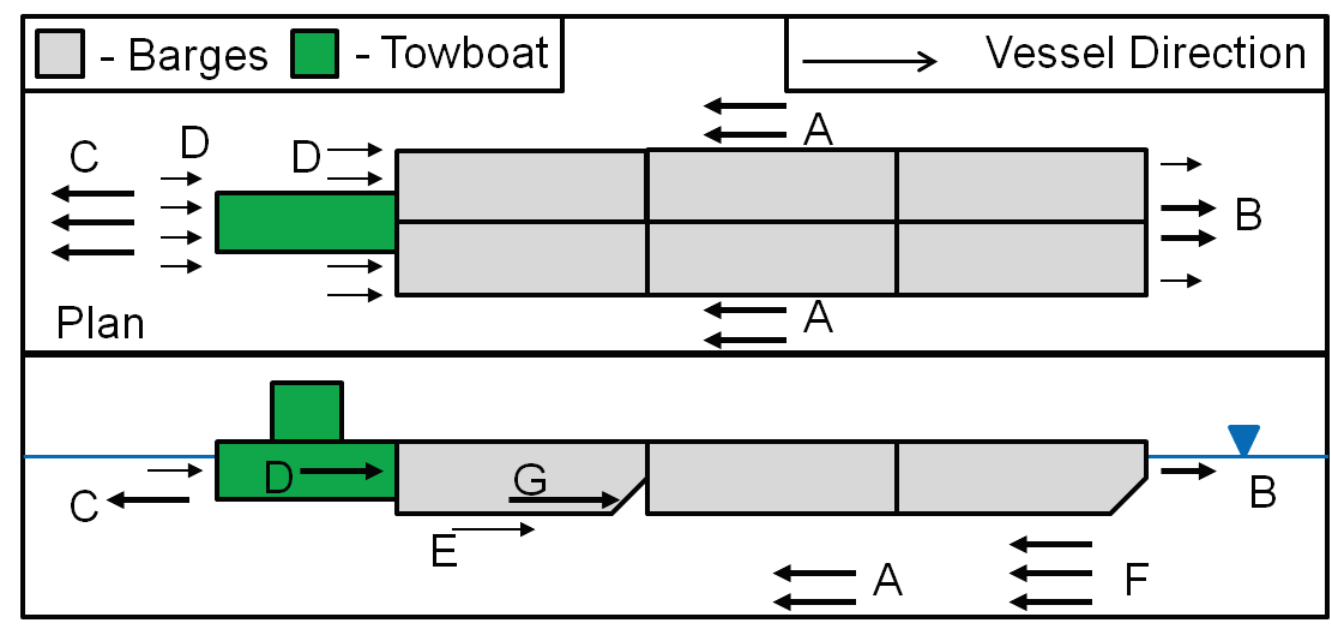

\subsubsection{Return velocity (A)}

When a vessel moves through a waterway, the hull displaces water that travels beside and beneath the vessel in the direction opposite to the direction of travel; water is basically moving from bow to stern. The flow moving opposite of the direction of travel is referred to as return flow or return velocity. In confined channels such as the CSSC, the return velocity tends to be relatively uniform over the cross section. In larger waterways, the return velocity is greatest at the tow and decays toward the bank. For upbound tows the return current increases the ambient velocities, and for downbound tows the return current decreases the ambient currents. In cases with strong return velocities and weak ambient flows, the net flow will temporarily reverse to an upstream direction. Maynord (1996) presents a method to determine return velocity based on tow speed relative to water, average channel depth, channel cross-section area, and tow cross-section area.

\subsubsection{Bow wave (B)}

Directly ahead of the tow, the displacement hull of the leading barge creates a bow wave, which creates a small packet of water that travels in 
the direction of the tow. For relatively shallow channels and relatively fastmoving vessels, bow flow exists over the full depth but is largest near the water surface. Bow flow occurs with raked (streamlined) barges but is greater for square- and blunt-nosed barges as leading barges. Away from the tow centerline, the flow wraps around the tow and contributes to the return velocity.

\subsubsection{Propeller jet (C)}

The propeller jet operates in the wake of the barges, causing a complex, turbulent flow field. The wake moves in the same direction as the tow, and the propeller jet moves opposite the tow. For high propeller speeds in shallow waterways, the jet can reach the channel bottom. Lower propeller speeds (typical of a no-wake restriction) may produce weak jets that do not extend to the channel bottom. For these lower propeller speeds, near-bed velocities under the propeller jet will be dominated by the wake flow and will be in the same direction as the tow. Outside the width of the propeller jet and behind the barges, the wake flow will be the dominant mechanism, and velocity will be in the same direction as the tow movement.

\subsubsection{Wake flow (D)}

The wake zone occurs directly aft of the barges and the towboat. Within the wake zone, water follows the tow at approximately the speed of the tow. The abrupt change in geometry between the last barge and the towboat likewise produces a barge wake zone and associated eddies that are carried with the tow. Wake effects are more pronounced with squareended barges but also exist with raked transitions. With the towboat centered on 2-wide barges, two corners or pockets exist where the wake flow moves along with the tow. The dynamics of the wake flow are governed by the local geometry and vessel speed.

\subsubsection{Vessel boundary layer (E)}

Vessels traveling through viscous fluids such as water develop a boundary layer adjacent to the hull. The thickness and other characteristics of this boundary layer depend on the vessel speed, hull roughness, channel geometry, and the CSSC discharge. The no-slip boundary condition at the hull causes the water adjacent to the hull to move at the same speed and direction as the tow. 


\subsubsection{Displacement velocity $(\mathbf{F})$}

Beneath the leading bow of the tow, a strong current is generated opposite the direction of tow travel, called the displacement velocity. Displacement flow is present beneath the bow of the full width of the barges and is relatively uniform between the hull and the bed.

\subsubsection{Barge junction recesses (G)}

The junction of two raked barges or the junction of a square-end barge and a raked barge forms a protected area with weak, nearly closed circulation. Water in these junctions may form a vertical roller or eddy that is similar in diameter to the barge draft. As with wake flow, the exchange of water, debris, and fish in and from this recess is controlled by barge speed, local geometry, turbulence, and floating object characteristics. In addition to junctions between barges, a junction is also present between the bow of the towboat and the stern of the barges. The junction or pocket between the barge and towboat may be particularly significant because the cavity or pocket is also within the wake of the barges.

\subsubsection{Drawdown}

Drawdown is the lowering of the water level adjacent to the tow between the tow and the bank. As with return velocity, drawdown is relatively uniform across the channel width for a channel as small as the CSSC. Drawdown is a big factor in defining the squat of a vessel. Due to its similarity to return velocity, drawdown is incorporated with return velocity for the remainder of this report.

\subsubsection{Prototype measurements}

Measurements (Maynord 2000) of near-bed velocity beneath a model tow (Figure 2-7) are helpful in illustrating the flows described above. In this example, a $9 \mathrm{ft}$ draft tow is traveling upstream in $23 \mathrm{ft}$ depth in a physical model of the upper Mississippi River (dimensions provided are at prototype scale). These depths and drafts are similar to the CSSC. Velocity and time in Figure 2-7 have been scaled to the prototype-scale system (see Section 3.1 for a similitude overview), and positive velocities indicate downstream flow. Ambient, near-bed velocity before the barge arrives (time $<0$ ) is approximately $0.7 \mathrm{ft} / \mathrm{s}$. At time zero, the bow of the tow is over the gage, and a short upstream pulse associated with the bow wave (mode B) is measured. Between $\mathrm{O}$ and $15 \mathrm{~s}$, the displacement velocity (F) 
strengthens downstream flow to approximately $3.3 \mathrm{ft} / \mathrm{s}$. Between 15 and $90 \mathrm{~s}$, the barges pass over the current meter generating a return current (A), which strengthens the downstream flow to approximately $1.6 \mathrm{ft} / \mathrm{s}$. At approximately $110 \mathrm{~s}$ the stern of the towboat passes over the meter, and between 120 and $300 \mathrm{~s}$ the current meter measures flow in the propeller jet (C). The near-bed propeller jet velocities are much weaker than those exiting the propellers due to the $23 \mathrm{ft}$ depth.

Figure 2-7. Near-bed velocities measured in $23 \mathrm{ft}$ water depth beneath barges and towboat (after Maynord 2000).

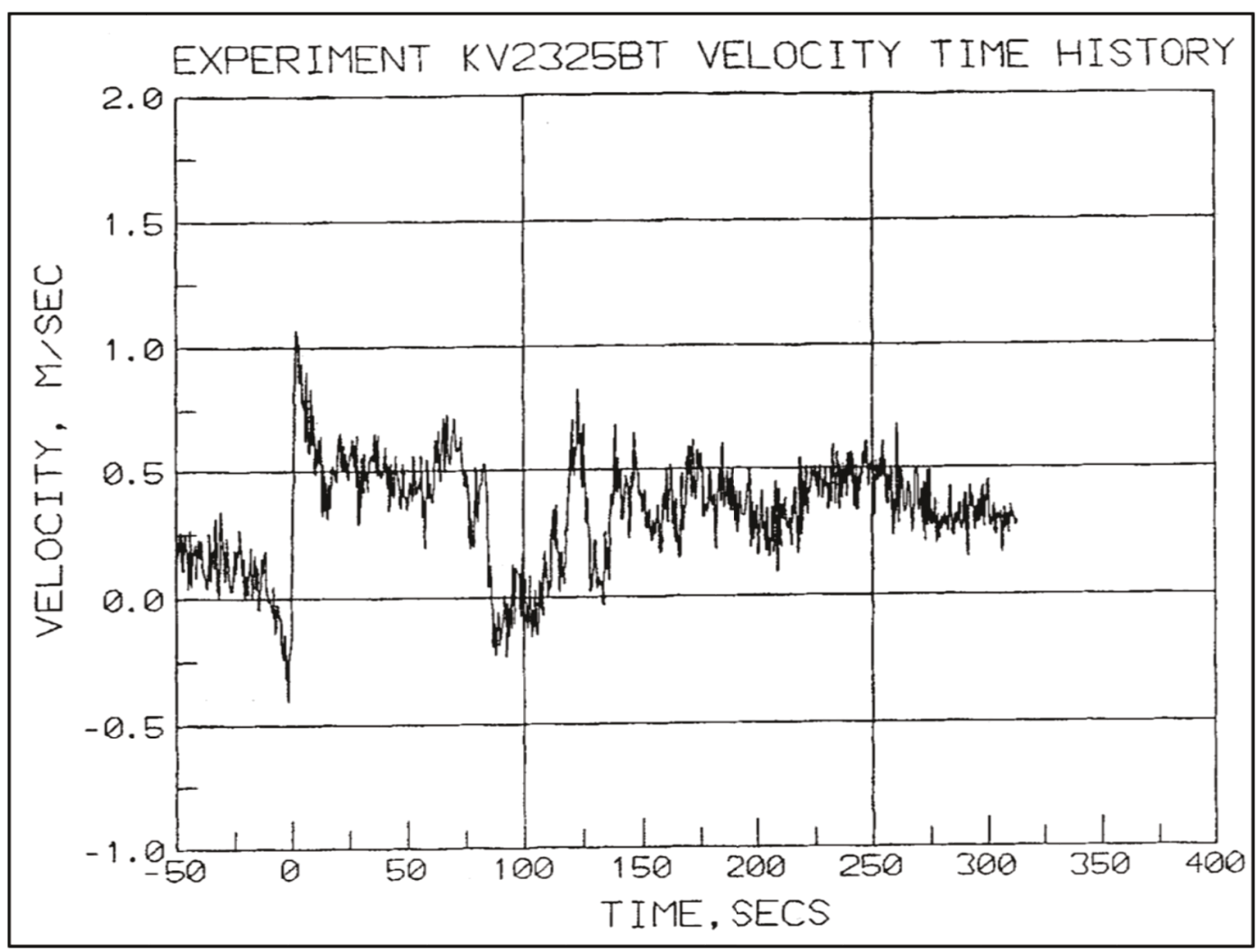




\section{Methods}

\subsection{Similitude overview}

To ensure the proper water motions around the tow, the model was geometrically similar and operated with the same Froude number, $F_{r}$, as the prototype, or

$$
F r=\frac{V_{M}}{\sqrt{g L_{M}}}=\frac{V_{P}}{\sqrt{g L_{P}}}
$$

where $V$ is the velocity, $g$ is gravitational constant, and $L$ is characteristic length scale. Geometric similarity means that the length in the prototype scale $(L P)$ is equal to the length ratio $(L r)$ of the model multiplied by the length in the model. The length ratio, $L r$, is defined as the prototype length divided by the model length. Table 3-1 describes how model parameters relate to the prototype through the length ratio $\left(L_{r}\right)$.

Table 3-1. Scaling of parameters from model to prototype scale.

\begin{tabular}{|c|c|c|c|}
\hline Parameter & Relationship to $\mathrm{Lr}_{\mathrm{r}}$ & $\begin{array}{c}\text { Ratio: Magnitude in } \\
\text { Prototype/Magnitude } \\
\text { in Model }\end{array}$ & Example \\
\hline $\begin{array}{l}\text { Any length } \\
\text { dimension }\end{array}$ & $L_{r}$ & 16.7 & $\begin{array}{l}1.68 \mathrm{ft} \text { water depth in } \\
\text { model = } 28 \mathrm{ft} \text { water } \\
\text { depth in full scale }\end{array}$ \\
\hline $\begin{array}{c}\text { Velocity of water or } \\
\text { tow speed }\end{array}$ & $\left(L_{r}\right)^{1 / 2}$ & 4.1 & $\begin{array}{l}1.0 \mathrm{mph} \text { tow speed in } \\
\text { model }=4.1 \mathrm{mph} \text { tow } \\
\text { speed in full scale }\end{array}$ \\
\hline Weight or volume & $\left(L_{r}\right)^{3}$ & 4657 & $\begin{array}{l}\text { A fish having a volume of } \\
0.015 \text { in. }^{3} \text { in the model }= \\
70 \text { in. }^{3} \text { in full scale }\end{array}$ \\
\hline Discharge & $\left(L_{r}\right)^{2.5}$ & 1140 & $\begin{array}{l}1.6 \mathrm{ft}^{3} / \mathrm{s} \text { in model }= \\
1800 \mathrm{ft}^{3} / \mathrm{s} \text { in full scale }\end{array}$ \\
\hline
\end{tabular}

\subsection{Modeled channel}

\subsubsection{Scale}

The CSSC is a straight rectangular channel near the electric barrier. The CHL has a facility with a $10 \mathrm{ft}$ wide by $4 \mathrm{ft}$ deep rectangular flume that has a length of approximately $500 \mathrm{ft}$. Based on the CSSC width, available model towboat size, and available model barge sizes, a scale ratio of 1:16.7 
was selected to model the CSSC. The facility is shown in Figure 3-1. Based on the $10 \mathrm{ft}$ flume width, the model channel corresponded to $167 \mathrm{ft}$ width at the prototype scale compared to actual width of $160 \mathrm{ft}$. The $500 \mathrm{ft}$ flume length scaled to a prototype scale length of approximately 1.5 miles, which allowed for the model tows to reach the proper speed before entering the test section. The test section represents the electric barrier and was located in the middle of the flume. Glass sidewalls were included in the test section to allow for viewing flows beside and beneath the tow.

\subsubsection{Flow}

As described in Chapter 2, there are times when the CSSC has a net flow rate from north to south. Three flow conditions were evaluated in the model study: (1) no flow, (2) a flow representing the 5oth percentile of flow rates, and (3) a high flow representing the 95th percentile of flow rates. The medium and high flow rates scaled $0.56 \mathrm{ft} / \mathrm{s}$ and $1.34 \mathrm{ft} / \mathrm{s}$, respectively. These scaled velocities were calculated using the scale relationships presented in Table 3-1.

The channel velocity was calibrated with a current meter. The velocity at 16 points in the channel was collected to create the calibration curve. The flow meter was very stable $(+/-0.5$ liters/s) and setting a specific flow rate consistently produced a specific corresponding velocity. The Valeport Model 802 current meter output standard deviation in addition to current velocity. The maximum reported standard deviation was $0.03 \mathrm{ft} / \mathrm{s}$ with an average standard deviation of $0.018 \mathrm{ft} / \mathrm{s}$.

\subsubsection{Roughness}

Because the CSSC was made by blasting bedrock, the channel sides and bed are not perfectly smooth. To match any conditions that might impact channel hydraulics, roughness elements were added to the model channel. Corrugated metal was used along the bottom and sides of the channel to model the roughness. A clear corrugated plastic simulated roughness over the viewing windows in the testing area. Additionally, the electrodes and parasitics for Barriers 2A and 2B were modeled by adding metal rods and mesh to the bottom of the model channel as seen in Figure 3-1. 
Figure 3-1. Barriers $2 \mathrm{~A}$ and $2 \mathrm{~B}$ in model test facility at $\mathrm{CHL}$. Wide-array mesh in foreground denotes parasitic structure; linear, narrow arrays in background denote the electrodes.

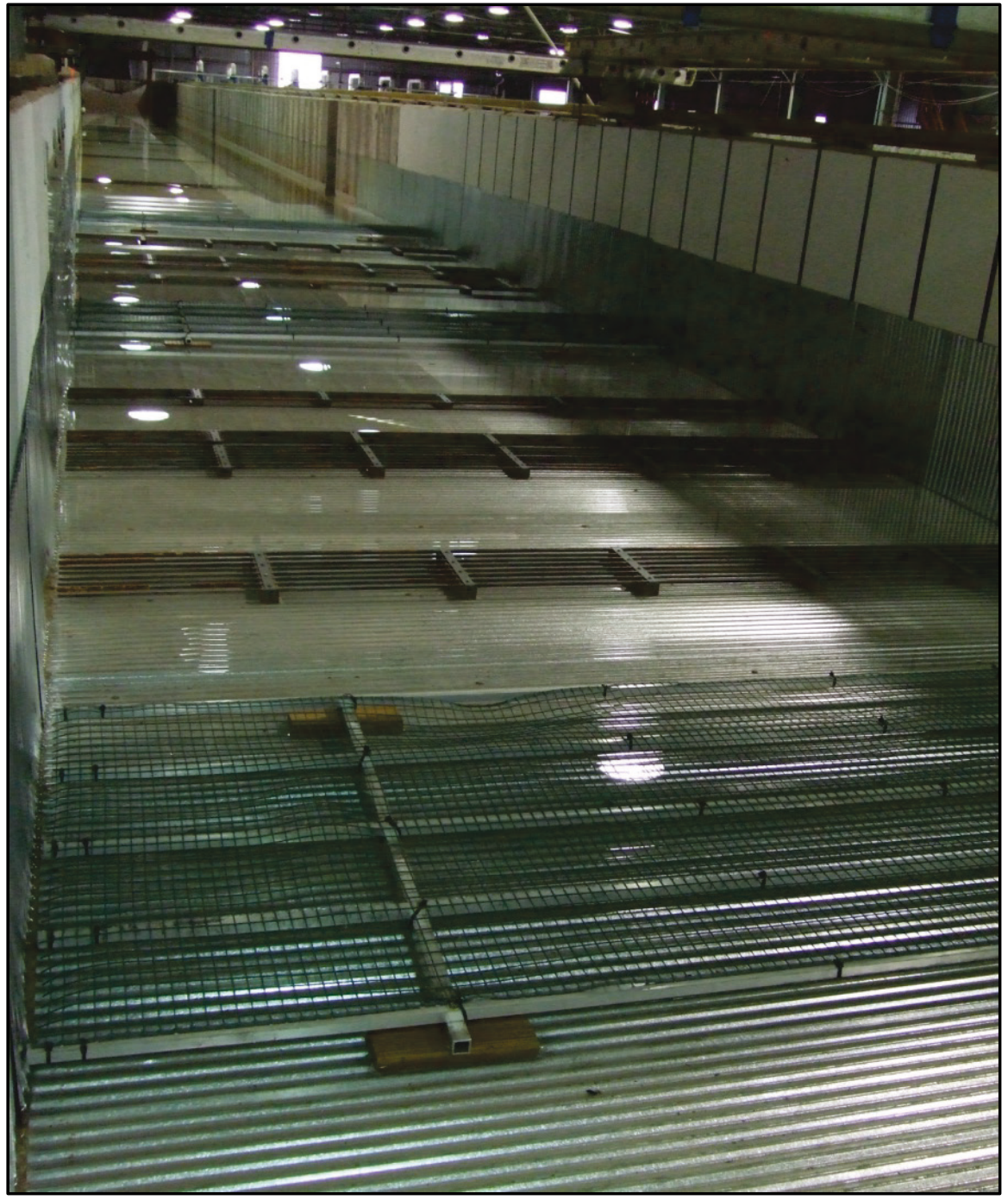

\subsection{Model barges}

Eight model barges were constructed of acrylic to allow observation and video recording of model fish movement mechanisms beneath the barges. Because of their large unloaded weight, the barges were only used to simulate loaded tows. Figure 3-2 shows a 2-wide by 3-long tow configuration moving through the model. The model barges were drafted to the desired $9 \mathrm{ft}$ by adding lead ingots and sandbags. Four of the barges have a rake on one end and a square end on the other while the other two barges have square ends on both ends of the barge. 
Figure 3-2. Asian carp test facility for navigation effects.

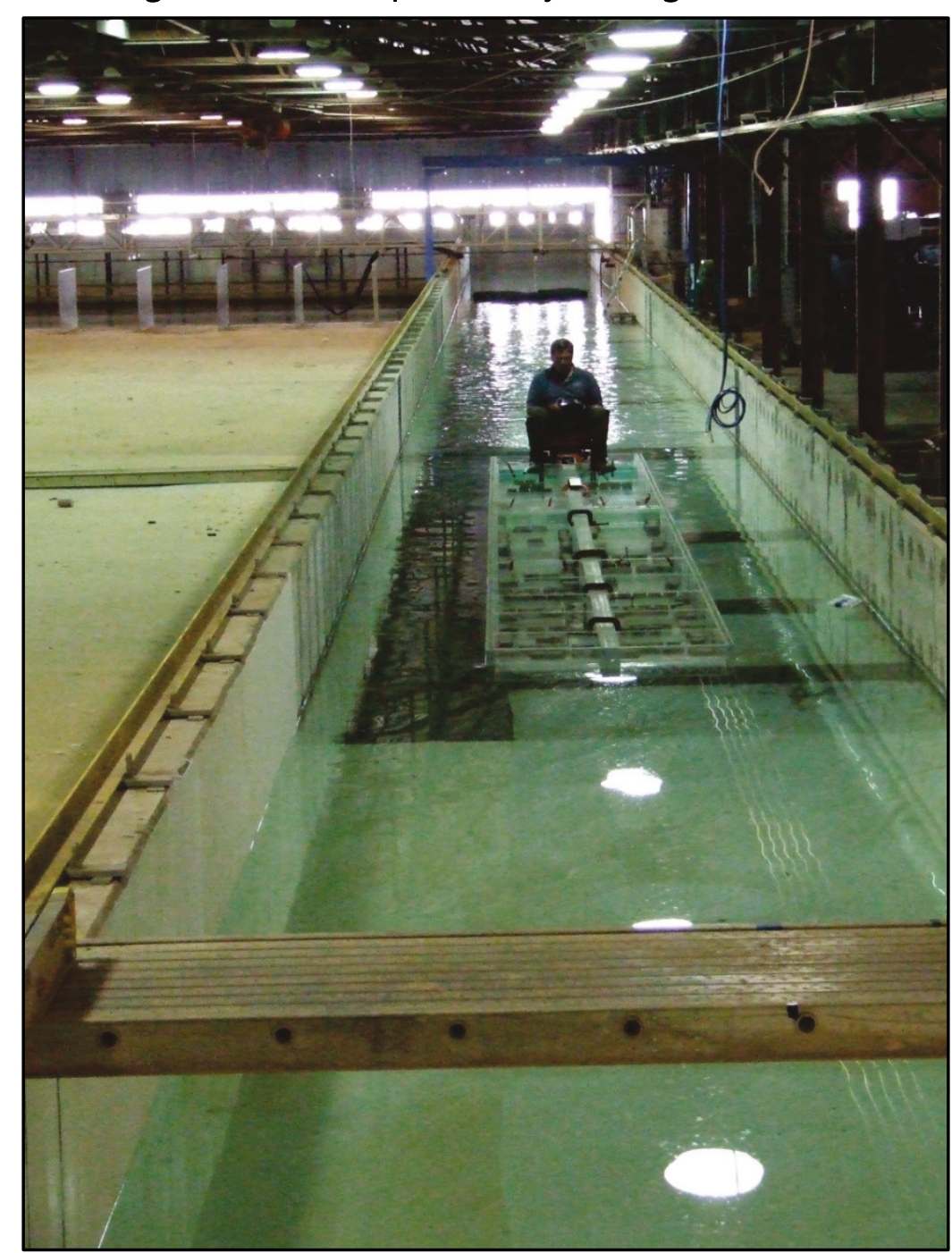

\subsubsection{Draft}

Scale effects arise because the physical size of the model is different from the full scale. The primary scale effect in navigation models is the difference between the boundary layer characteristics in the model and in the prototype as shown in Figure 3-3. The boundary layer is the zone of reduced velocity very near a boundary like a ship hull or a channel bottom. The velocity is zero at the boundary and a maximum at the edge of the boundary layer where the velocity is no longer reduced by the presence of the boundary. This causes larger frictional forces in the model than in the prototype. 
Figure 3-3. Schematic of boundary layer on hull and channel bottom for both model and full-scale tows.

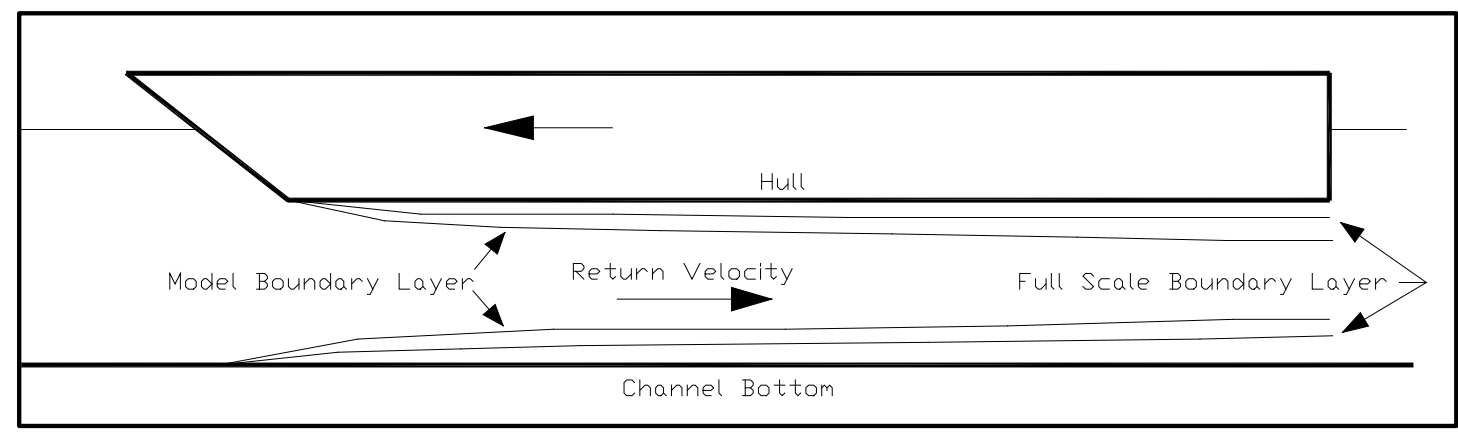

Physical modeling of ship motion requires adjustments to the model draft to better simulate the prototype draft. Maynord and Martin $(1997,1998)$ used the difference in model and prototype-scale displacement thickness to adjust model draft. The displacement thickness indicates the distance by which the streamlines outside the boundary layer are shifted by the formation of the boundary layer. The Prandtl-Schlichting skin friction equation for a smooth, flat plate at zero incidence (Schlichting 1968) produces the following equation:

$$
\delta_{1}=\frac{0.292 L}{\left[\log \left(R e_{L}\right)\right]^{2.58}}
$$

where $\delta_{1}=$ displacement thickness, $L=$ plate length set equal to length along barges, $R_{L}=$ plate Reynolds number defined as $V L / v, V=$ speed relative to water, and $v=$ kinematic viscosity of water. For the hull of a tow in a confined channel, $V$ is the sum of the tow speed over land and the return velocity.

The displacement thickness was calculated for the CSSC and the 2-wide by 3 -long tow having $9 \mathrm{ft}$ draft in $26 \mathrm{ft}$ water depth in the CSSC. The tow was moving at $4 \mathrm{mph}$ when there was no flow in the canal. The return velocity was determined using the Schijf (1949) equation, which is based on the one-dimensional application of conservation of energy and conservation of mass. Based on the Schifj equation, the return velocity was $2.1 \mathrm{ft} / \mathrm{s}$ for the 2-wide by 3 -long by $9 \mathrm{ft}$ draft barge flotilla at $4 \mathrm{mph}$ in the CSSC. The water temperature was $70^{\circ} \mathrm{F}$ such that $v=0.0000106 \mathrm{ft}^{2} / \mathrm{s}$. Results are listed in Table 3-2. The characteristic velocity used to calculate the Reynolds number for the hull boundary layer was the tow speed plus the return velocity. The characteristic velocity used for the Reynolds number for the bed boundary layer was the return velocity. 
Table 3-2. Displacement thickness calculations. Model scale $=1: 16.66$. Tow speed $=4 \mathrm{mph}$.

\begin{tabular}{|c|c|c|c|c|c|c|}
\hline Case & Location & $V, \mathrm{ft} / \mathrm{s}$ & $L, \mathrm{ft}$ & $R$ & $\delta_{1}, \mathrm{ft}$ & $\begin{array}{l}\delta_{1} \text { difference, model } \\
\text { - prototype-scale, } \mathrm{ft}\end{array}$ \\
\hline $\begin{array}{l}\text { Prototype- } \\
\text { scale hull }\end{array}$ & $\begin{array}{l}\text { End of } 1^{\text {st }} \\
\text { barge }\end{array}$ & $\begin{array}{l}5.87+2.1= \\
7.97\end{array}$ & 195 & $1.47(10)^{8}$ & 0.253 & - \\
\hline Model hull & $\begin{array}{l}\text { End of } 1^{\text {st }} \\
\text { barge }\end{array}$ & $\begin{array}{l}1.44+0.51= \\
1.95\end{array}$ & 11.7 & $2.16(10)^{6}$ & $\begin{array}{l}0.0292 * 16.7= \\
0.487\end{array}$ & 0.234 \\
\hline $\begin{array}{l}\text { Prototype- } \\
\text { scale bed }\end{array}$ & $\begin{array}{l}\text { End of } 1^{\text {st }} \\
\text { barge }\end{array}$ & 2.1 & 195 & $3.86(10)^{7}$ & 0.305 & - \\
\hline Model bed & $\begin{array}{l}\text { End of } 1^{\text {st }} \\
\text { barge }\end{array}$ & 0.51 & 11.7 & $5.68(10)^{5}$ & $\begin{array}{l}0.0374 * 16.7= \\
0.623\end{array}$ & 0.318 \\
\hline $\begin{array}{l}\text { Prototype- } \\
\text { scale hull }\end{array}$ & $\begin{array}{l}\text { End of } 2^{\text {nd }} \\
\text { barge }\end{array}$ & $\begin{array}{l}5.87+2.1= \\
7.97\end{array}$ & 390 & $2.93(10)^{8}$ & 0.460 & - \\
\hline Model hull & $\begin{array}{l}\text { End of } 2^{\text {nd }} \\
\text { barge }\end{array}$ & $\begin{array}{l}1.44+0.51= \\
1.95\end{array}$ & 23.4 & $4.31(10)^{6}$ & $\begin{array}{l}0.0518 * 16.7= \\
0.863\end{array}$ & 0.403 \\
\hline $\begin{array}{l}\text { Prototype- } \\
\text { scale bed }\end{array}$ & $\begin{array}{l}\text { End of } 2^{\text {nd }} \\
\text { barge }\end{array}$ & 2.1 & 390 & $7.73(10)^{7}$ & 0.552 & - \\
\hline Model bed & $\begin{array}{l}\text { End of } 2^{\text {nd }} \\
\text { barge }\end{array}$ & 0.51 & 23.4 & $1.14(10)^{6}$ & $\begin{array}{l}0.0656 * 16.7= \\
1.09\end{array}$ & 0.540 \\
\hline $\begin{array}{l}\text { Prototype- } \\
\text { scale hull }\end{array}$ & $\begin{array}{l}\text { End of } 3^{\text {rd }} \\
\text { barge }\end{array}$ & $\begin{array}{l}5.87+2.1= \\
7.97\end{array}$ & 585 & $4.40(10)^{8}$ & 0.654 & - \\
\hline Model hull & $\begin{array}{l}\text { End of } 3^{\text {rd }} \\
\text { barge }\end{array}$ & $\begin{array}{l}1.44+0.51= \\
1.95\end{array}$ & 35.1 & $6.47(10)^{6}$ & $\begin{array}{l}0.0727 * 16.7= \\
1.21\end{array}$ & 0.556 \\
\hline $\begin{array}{l}\text { Prototype- } \\
\text { scale bed }\end{array}$ & $\begin{array}{l}\text { End of 3rd } \\
\text { barge }\end{array}$ & 2.1 & 585 & $1.16(10)^{8}$ & 0.783 & - \\
\hline Model bed & $\begin{array}{l}\text { End of } 3^{\text {rd }} \\
\text { barge }\end{array}$ & 0.51 & 35.1 & $1.70(10)^{6}$ & $\begin{array}{l}0.091 * 16.7= \\
1.52\end{array}$ & 0.739 \\
\hline
\end{tabular}

The difference in displacement thickness (see Table 3-2) is greater on the bed than on the hull, and this difference varies along the length of the tow. A more advantageous result would be if the bed dissimilarity could be addressed by an adjustment of the depth, but that could not be achieved because of the variation of bed boundary layer effect from zero at the bow to the maximum at the stern of the tow. The approach used in this study was to combine the dissimilar bed and hull boundary layer effect into an adjustment of the draft of the tow to obtain the correct boundary layer effects beneath the tow, the correct blockage ratio, and correct return velocity. This method was used by Maynord and Martin $(1997,1998)$. In those studies, the model draft was kept uniform for all barges, and the draft was varied until the model return velocity was equal to the return velocity measured in the field for the same scale tow configuration (five barges long), tow speed, and channel cross section. At the 1:25 scale, the uniform 
model draft to simulate $9 \mathrm{ft}$ draft was found to be approx-imately $7.25 \mathrm{ft}$. In the CSSC study, keeping the bow correctly drafted to ensure that boundary layer scale effects were not present was important to the movement of model fish beneath the tow. In this study, the model draft was adjusted from zero (at the bow) to the sum of the bed and hull dissimilarity (at each barge junction along the tow). At the end of the first barge, the sum of the bed and hull dissimilarity was $0.234 \mathrm{ft}+0.318 \mathrm{ft}=0.552 \mathrm{ft}$.

A variable draft was applied in the model to address scale dissimilarities at the hull and the bed. A draft of $9 \mathrm{ft}$ at the bow of the first barge decreasing to $7.7 \mathrm{ft}$ at the stern of the last barge was consistent with the approach of Maynord and Martin (1997, 1998).

\subsubsection{Roughness}

The model barges used in this study were made of acrylic, which has very low roughness. However, real barges have rust and surface indentations from service. The roughness of a barge greatly influences the growth and size of the boundary layer and subsequent mixing. Generally, greater surface roughness increases mixing in the boundary layer. The previous model study by Bryant et al. (2016) investigated the effects of barge roughness by wrapping the leading barges in a plastic grid with 1 in. spacing and a thickness of 0.2 in. This spacing was equivalent to a prototype roughness of $3.34 \mathrm{in}$. The results of this study showed that the additional barge roughness increased the subsequent boundary layer and increased the return current. The use of unmodified smooth barges in this study allowed for the results to be compared to those of previous testing under the same barge conditions.

\subsubsection{Propulsion}

As in the prototype channel, the model barges were pushed by a model towboat. The CHL has a remote-controlled towboat that is scaled based on the USACE MV Benyaurd. The model towboat is $6.8 \mathrm{ft}$ long by $1.6 \mathrm{ft}$ wide and has twin, open-wheel propellers.

\subsubsection{Speed}

The speed of the model barges was measured by timing the period that the barges took to pass through a given section of known length. With a known distance and time, speed was calculated. A tachometer displayed the 
revolutions per minute (rpm) for the propellers, and these readings were used as a guide for the boat operator to set a desired vessel speed. Multiple tests were conducted to determine the correct rpm reading needed to achieve a speed. Speed was calculated for each test run either during testing with the previously described method or after the test's completion by reviewing camera footage.

Speed is an important parameter as it impacts both the magnitude and duration of the return current as well as the effectiveness of jet flushing in the rake. As discussed in Chapter 2, speed information was collected using the AIS data, and this data provided the guidelines for which speeds were tested in the model study. Two speeds were chosen as a representative range of speeds for barge traffic in the area of the barriers, $2.5 \mathrm{mph}$ and $5 \mathrm{mph}$.

\subsubsection{Maneuvers}

The model towboat was fitted with twin, open-wheel propellers. The remote control had dual controls that allowed the two propellers to be operated independently. The independent control of the propellers provided the model barges with more maneuverability to reproduce maneuvers of the prototype vessel.

\subsection{Modeled fish}

\subsubsection{Fish similitude and scaling}

When conducting a physical model study of open channel flows, matching the Froude number, Fr, guarantees dynamic similitude. However, objects submerged in the flow, such as passive tracers, can be characterized by two different parameters, the Reynolds number and the Stokes number.

The Reynolds number is the ratio of the inertial forces to the viscous forces. When the Reynolds number is small, the flow is described as laminar $(\operatorname{Re}<2000)$. When the Reynolds number is large, the flow is turbulent $(\operatorname{Re}>4000)$. If the prototype fluid is used in a model study, matching both the Froude and Reynolds numbers is impossible. However, turbulence is self-similar at small scales. If the prototype is turbulent, then ensuring that the model is also fully turbulent is the most important detail. 
The Stokes number is the ratio of the force needed to accelerate or decelerate a particle of mass $(\mathrm{m})$ to the force of the flow. The Stokes number is typically defined as

$$
S t=\frac{\tau_{s}}{\tau_{\eta}}
$$

where $\tau_{s}$ is the relaxation time of the particle and $\tau_{\eta}$ is the time-scale of the fluid (Bec et al. 2006). A particle with a $S t>>1$ represents a particle that will not move with the flow whereas a $S t<<1$ means the particle will move seamlessly with the flow. Particles with a Stokes number near 1.0 represent a difficult regime to model and study since they may move with and against the flow in time.

The relaxation time is given by

$$
\tau_{s}=\frac{2 \rho_{p} a^{2}}{9 \rho_{f} \nu}
$$

where $\rho_{s}$ is the particle density, $a$ is the particle radius, and $\rho_{f}$ is the fluid density. The time-scale of the fluid is typically given as

$$
\tau_{\eta}=\left(\frac{v}{\varepsilon}\right)^{1 / 2}
$$

where $\varepsilon$ is the eddy diffusivity. This fluid time-scale is equivalent to the Kolmogorov time-scale, the time-scale of the smallest eddies before viscous dissipation. This term is difficult to measure, and often a substitute must be found. One method to find a substitute is to define the scale of fluid motion that is desired for the particles to follow. When considering the interaction between ship traffic and stunned fish, the largest scale fluid motion is equivalent to the barge draft $(d b)$, and the smallest scale is that of the fish length $\left(d_{f}\right)$. Using these two length scales, and the characteristic eddy velocity $\left(V_{e}\right)$, the relaxation time can be confined to

$$
\frac{d_{f}}{V_{e}} \leq \tau_{\eta} \leq \frac{d_{b}}{V_{e}}
$$


As with the Reynolds number, matching the Stokes number and Froude number simultaneously is often either impossible or impractical, but having the particles within the same regime is possible. The Stokes number of the prototype fish is much larger than 1.o, so the model fish must also have a Stokes number much larger than 1.o. This Stokes number requirement limits the size of model fish that can be used, but it ensures the proper dynamic response of the fish.

\subsubsection{Fish size}

Rubber fishing lures were used to represent the Asian carp in the model study. To determine a suitable model fish size, the density and length scale of the prototype fish must first be determined. Stunned fish are nearly neutrally buoyant, so for this analysis, the prototype fish are assumed to have a density equivalent to the water density. Field observations show that electrically-stunned fish float to the surface. The length of the fish is an easily identifiable length scale used in place of the particle diameter. This substitution reduces the Stokes number to

$$
\frac{d_{f} V_{e}}{18 v d_{f}} \leq S t \leq \frac{d_{f} V_{e}}{18 v d_{b}}
$$

For comparisons, the prototype velocity ranged from $0.3 \mathrm{ft} / \mathrm{s}$ to $3.3 \mathrm{ft} / \mathrm{s}$ at $0.3 \mathrm{ft} / \mathrm{s}$ intervals. This velocity was calculated for the model based on Froudian similitude. The depth of the barge draft was taken as $9.2 \mathrm{ft}$ for the prototype and $0.56 \mathrm{ft}$ for the model based on the geometric similitude. LRC requested that the prototype fish length considered in this investigation be $8 \mathrm{in.} \mathrm{long,} \mathrm{and} \mathrm{for} \mathrm{the} \mathrm{model} \mathrm{fish,} \mathrm{a} \mathrm{possible} \mathrm{range} \mathrm{was}$ selected of 0.5, 1.0, 2.0, 3.0, and 4.0 in. Figure 3-4 shows, on a log-log scale, the comparison of the prototype Stokes number and model Stokes number for a fish-sized eddy and the velocity ranges selected. The solid line represents a match between the model and prototype Stokes number. The smaller model fish of 0.5 in. and 1.0 in. have much smaller Stokes numbers compared with the prototype. These smaller eddies had difficulty moving a fish of the equivalent size. However, Figure 3-5 shows the comparison between the model and prototype fish for eddies that are the same length scale as the barge draft. 
Figure 3-4. Comparison of prototype fish Stokes number to model fish Stokes number for a fish-size eddy.

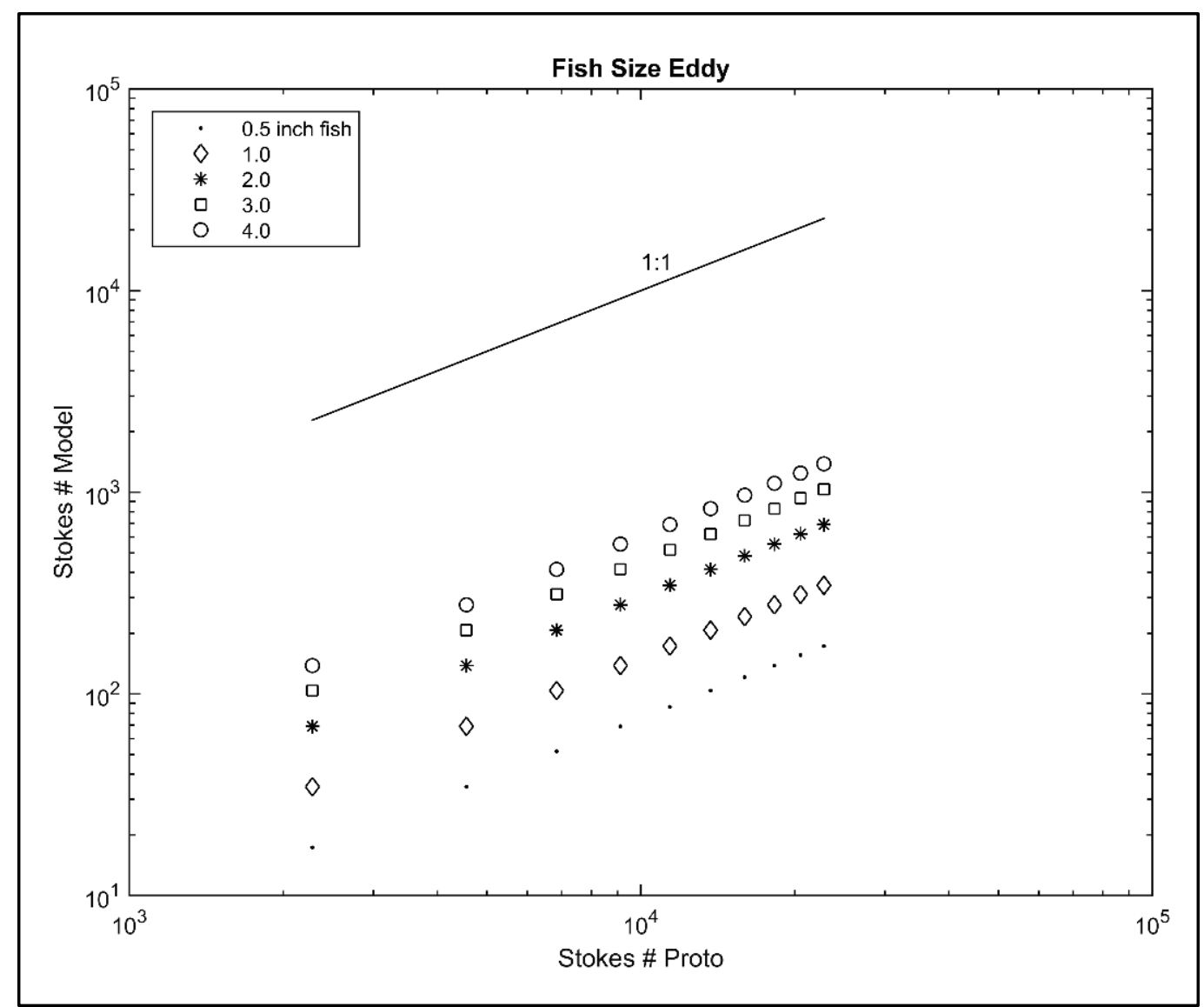

Figure 3-5 shows that the scaling factors of the smaller 0.5 in. and 1.0 in. model fish are very close to 1.00 at lower velocities and may not act similarly to the prototype fish. Also, geometric similitude and Stokes similitude could not be achieved for the smaller prototype fish. However, by using larger model fish, the transport of fish by a passing barge can still be determined. A range of model fish was investigated as the eddy length scale is different depending on the mode of transport. 
Figure 3-5. Comparison of prototype fish Stokes number to model fish Stokes number for a barge draft size eddy.

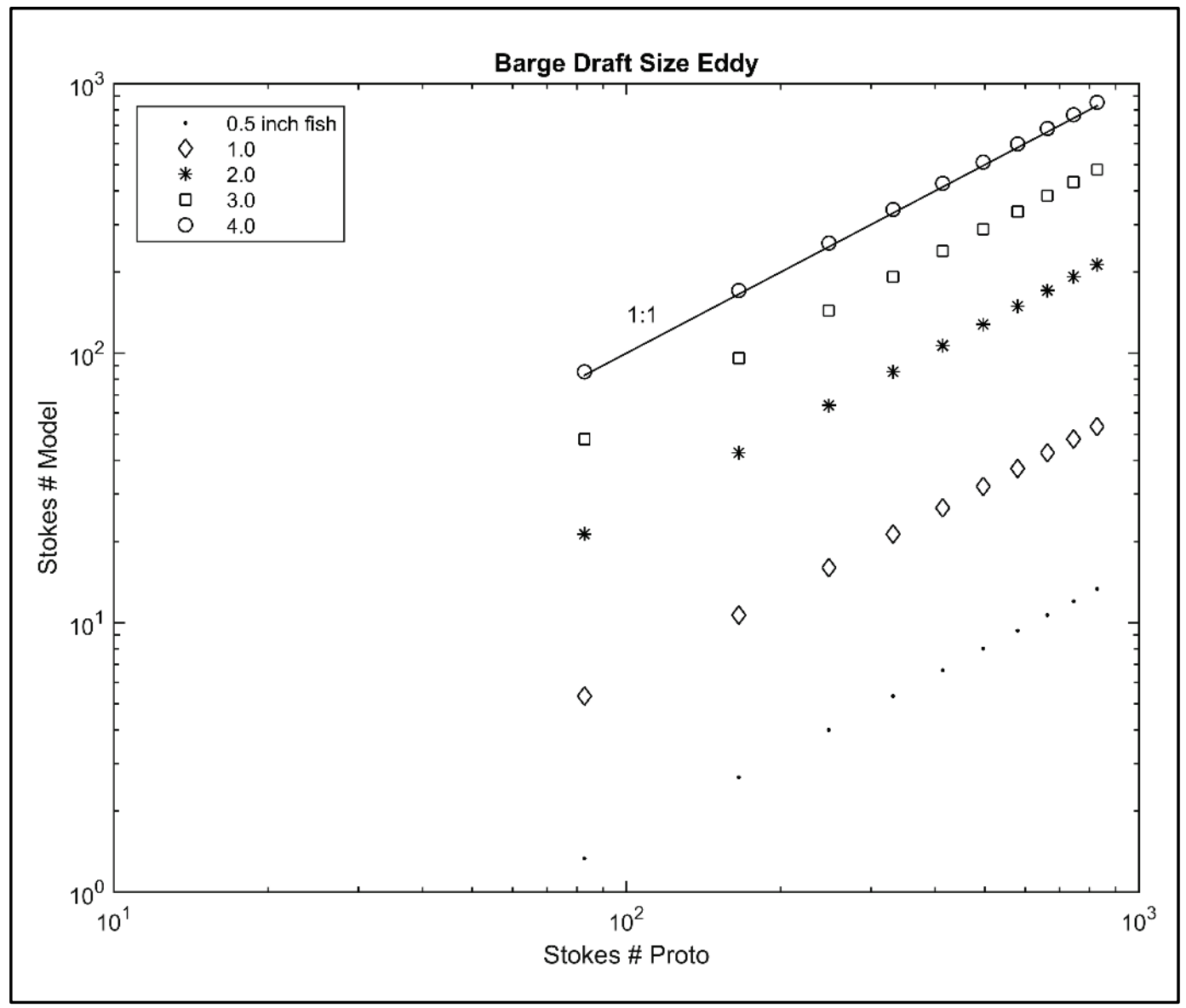

Figure 3-6 shows the Stokes comparison for the wake behind the barges with a vortex length scale (barge width) estimated at $35 \mathrm{ft}$. Here, the prototype Stokes number was much smaller, so a smaller-scaled model fish could be used. This analysis led to the conclusion that a range of model fish sizes should be used. The use of very large model fish would have presented challenges when reducing fish-to-fish interaction, with the model fish being larger than the barge-generated vortices due to the change in Reynolds number and geometric scaling. Three model fish sizes with lengths of $1.5 \mathrm{in} ., 1.875 \mathrm{in}$., and $2.25 \mathrm{in}$. were determined to adequately represent Asian carp (Figure 3-7). Closed-core foam and fine copper wire were used to modify the model fish density, making them close to neutrally buoyant. 
Figure 3-6. Comparison of prototype fish Stokes number to model fish Stokes number for a barge width-size eddy.

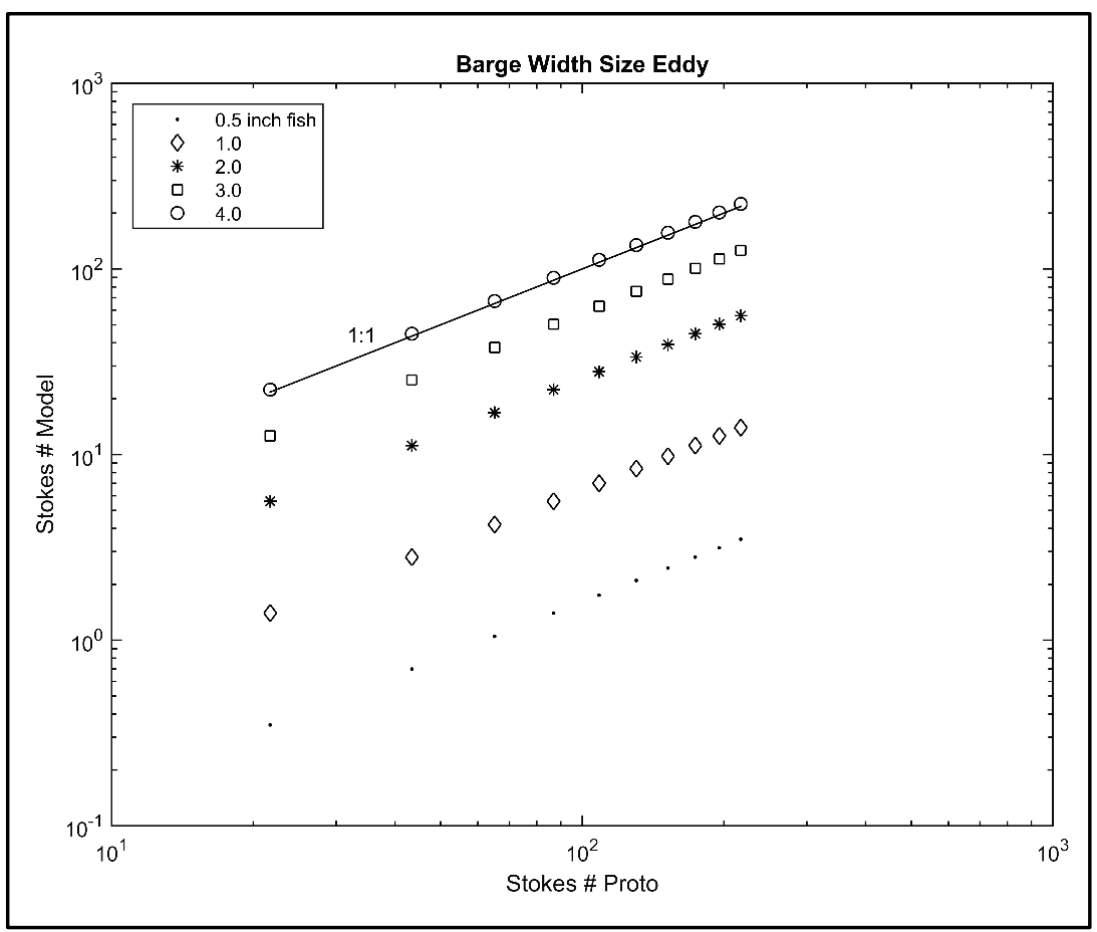

Figure 3-7. Comparison of three model fish with lengths of $1.5 \mathrm{in}$., 1.875 in., and 2.25 in.

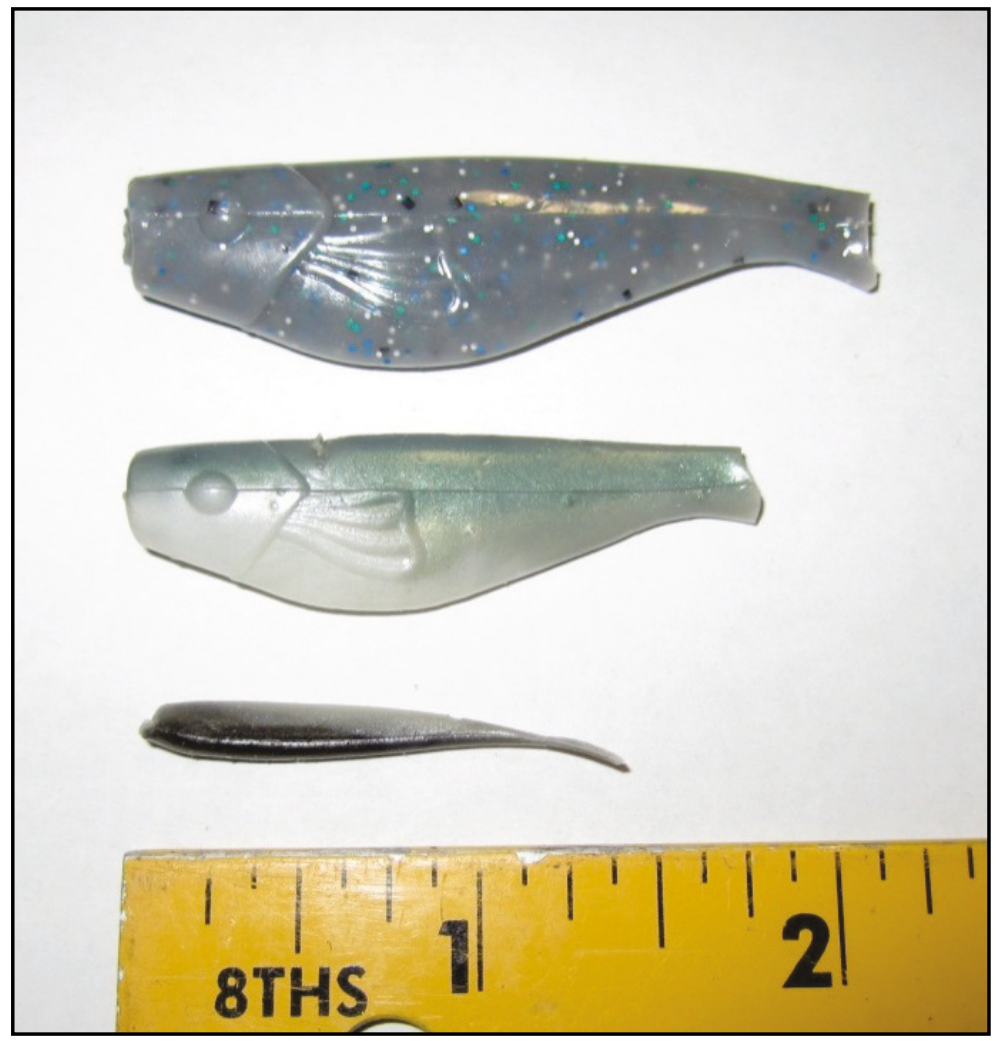




\subsection{Instrumentation}

During testing, multiple flow measurement devices and video equipment were used to aide in recording data for analysis as well as taking real-time measurements. The following instrumentation was used during this study.

\subsubsection{Acoustic Doppler Velocimetry (ADV)}

An ADV is an instrument placed into the water column that provides highresolution velocity measurements in three dimensions. To measure the magnitude and duration of the return current, an array of three Nortek Vectrino ADVs were placed in the model channel. The array of ADVs measured the velocities in the channel at three distances from the wall simultaneously. Also, the mount could be raised and lowered, so velocity measurements could be taken at different water column depths. The ADVs also measured the ambient flow in the model channel for tests where a flow condition was modeled.

\subsubsection{Ultrasonic flow meters}

An EESIFLO Sonalok 5000 Series transit time ultrasonic flow meter was attached to the $8 \mathrm{in}$. diameter discharge pipe of the $5 \mathrm{cfs}$ centrifugal pump used to produce flow in the channel. Measurements are made by penetrating the pipe with ultrasound, and subsequently, time differences, frequency variations, and phase shifts of the ultrasonic signals are evaluated. This non-invasive method has no effect on the flowing fluid. The instrument has a stated accuracy of $+/-3 \%$ of the measured flow volume.

\subsubsection{Electromagnetic current meter}

A Valeport model 802 2-axis electromagnetic current meter was used to measure channel flow. The sensor uses the Faraday principle to measure flow past the sensor in two orthogonal axes. The magnetic field is generated within the sensors by a coil, and the electronics detect the signal generated across a pair of electrodes on each axis. The sampling mode used was a $30 \mathrm{~s}$ fixed average.

\subsubsection{Cameras}

An Allied Vision Technologies Prosilica GT color camera was mounted from the ceiling to record the maneuver testing. The camera was also mounted on the railing to record the return current and jet flushing tests. 
The camera had a resolution of 1,624 pixels by 1,234 pixels and was operated from a desktop through the StreamPix recording software.

Two GoPro Hero4 Black cameras were mounted in the barge and were positioned to capture images of the rake void area during testing. The cameras were operated at 108op resolution. Both cameras were controlled remotely with the GoPro mobile application.

\subsection{Jets}

\subsubsection{Manifold}

The hydraulic manifold consisted of 23 evenly spaced 1 in. schedule ( $\mathrm{SCH}$ ) 40 polyvinyl chloride (PVC) outlets mounted in a metal base (Figure 3-8). The outlets were designed to accommodate multiplesized fittings for the nozzles. The intake was located at the center of the manifold and delivered uniform flows to each outlet. Visual inspection showed some decrease in upwelling along the manifold, but this was found to be so small that for testing purposes the assumption of uniform flow was not violated. After completing the slow vessel speed tests, two additional manifolds were constructed to increase the length of the array and the total number of jets. The pumping system was modified to accommodate the greater discharge so that replicating the single manifold jet velocities was possible with the longer array.

Figure 3-8. Single manifold placed in the center of the channel parallel to the channel walls.

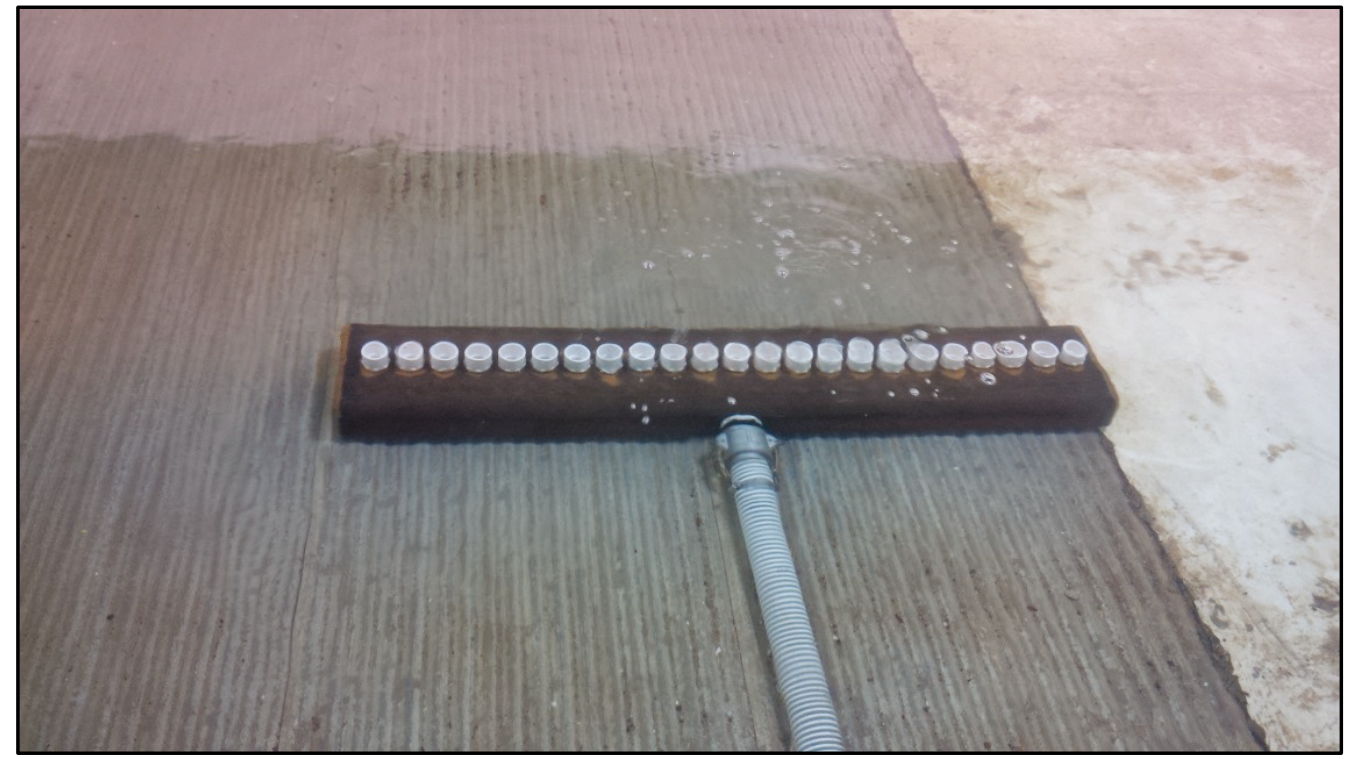




\subsubsection{Nozzles}

The jet nozzles were modeled with SCH 40 PVC fittings that could be attached and removed from the jet manifold (Figure 3-9). The nozzle fittings varied in diameter and angle. The model diameters used in the study were $0.5,0.75$, and $1 \mathrm{in}$. fittings corresponding to prototype diameters of 8,12 , and 16 in., respectively. The angles used were 45 degrees (deg) and 22.5 deg from the vertical with a straight fitting for vertical jet tests. A 45 deg fitting was attached to a 22.5 deg fitting to form a jet nozzle with a total angle of $67.5 \mathrm{deg}$ from the vertical. The jets were attached such that the nozzle's horizontal angle from the centerline could be varied for different jet configurations.

Figure 3-9. Manifold with 8 in. (prototype) nozzles. Nozzles are oriented at $45 \mathrm{deg}$ off of the vertical and are rotated in an alternating pattern to force fluid obliquely to the path of the tow as it passes overhead.

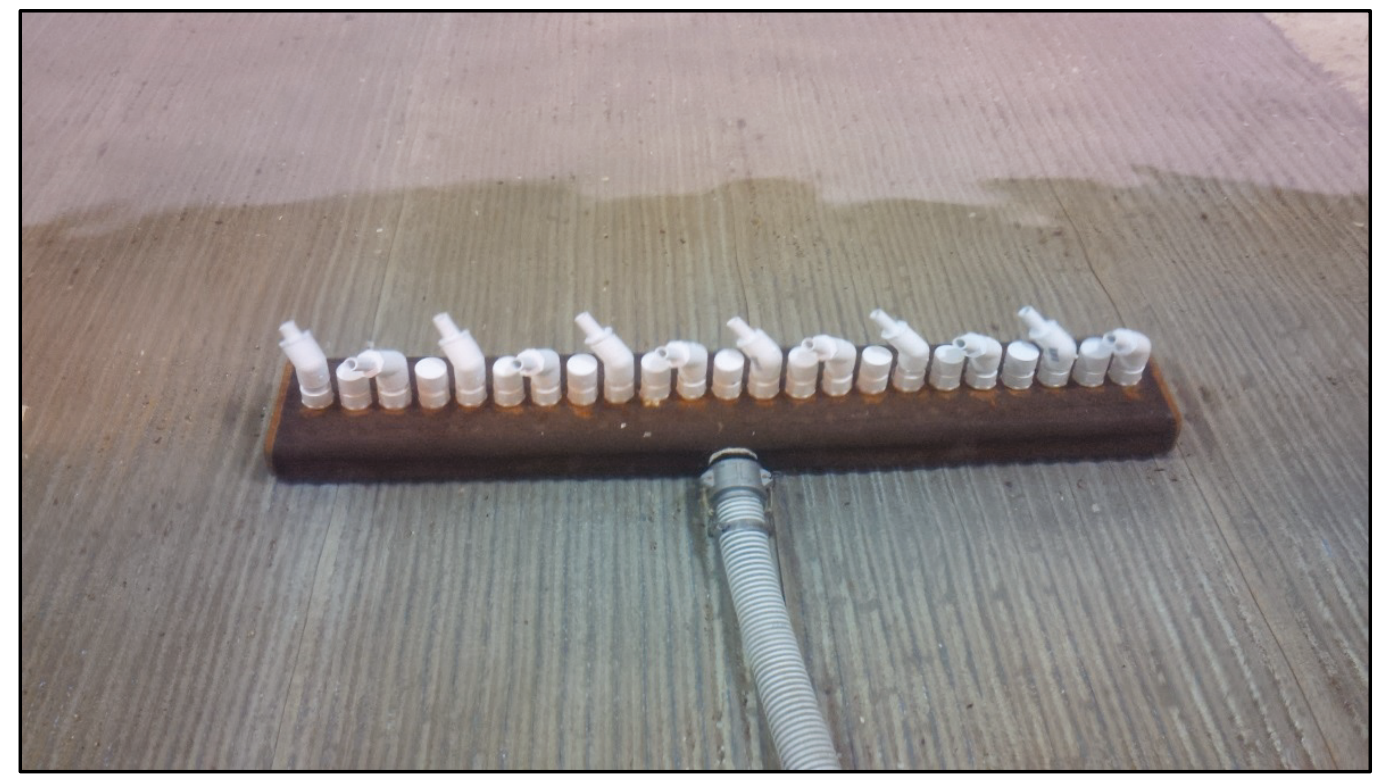

\subsubsection{Jet array terminology}

A variety of jet angles and configurations were tested throughout the study according to the variables listed below. Figure 3-10 shows how and where each variable is measured in the manifold and jet configuration.

Nozzle diameter, $\mathbf{D}$ : The inner diameter of the opening of the jet nozzle. Diameters of 8 in., 12 in., and 16 in. in prototype scaling were tested.

Nozzle spacing, ls: The space between jet nozzles measured from the nozzle center to adjacent nozzle center. 
Jet array length, L: The length from the first nozzle center to the last nozzle center.

Jet height, H: The height of the jet nozzle opening measured from the channel bottom including the height of the manifold.

Horizontal angle, $\boldsymbol{y}$ : The angle of the jet nozzle alignment relative to the channel centerline.

Vertical angle, $\boldsymbol{\alpha}$ : The angle of the jet nozzle alignment relative to the vertical axis. Angles of o deg, $45 \mathrm{deg}$, and $67.5 \mathrm{deg}$ were tested.

Manifold angle, $\boldsymbol{\beta}$ : The angle of the jet manifold alignment relative to the channel centerline.

Jet flow rate, Qjet: The total flow rate supplied to the jet manifolds.

Figure 3-10. Nozzle and jet variables for jet and manifold configurations.

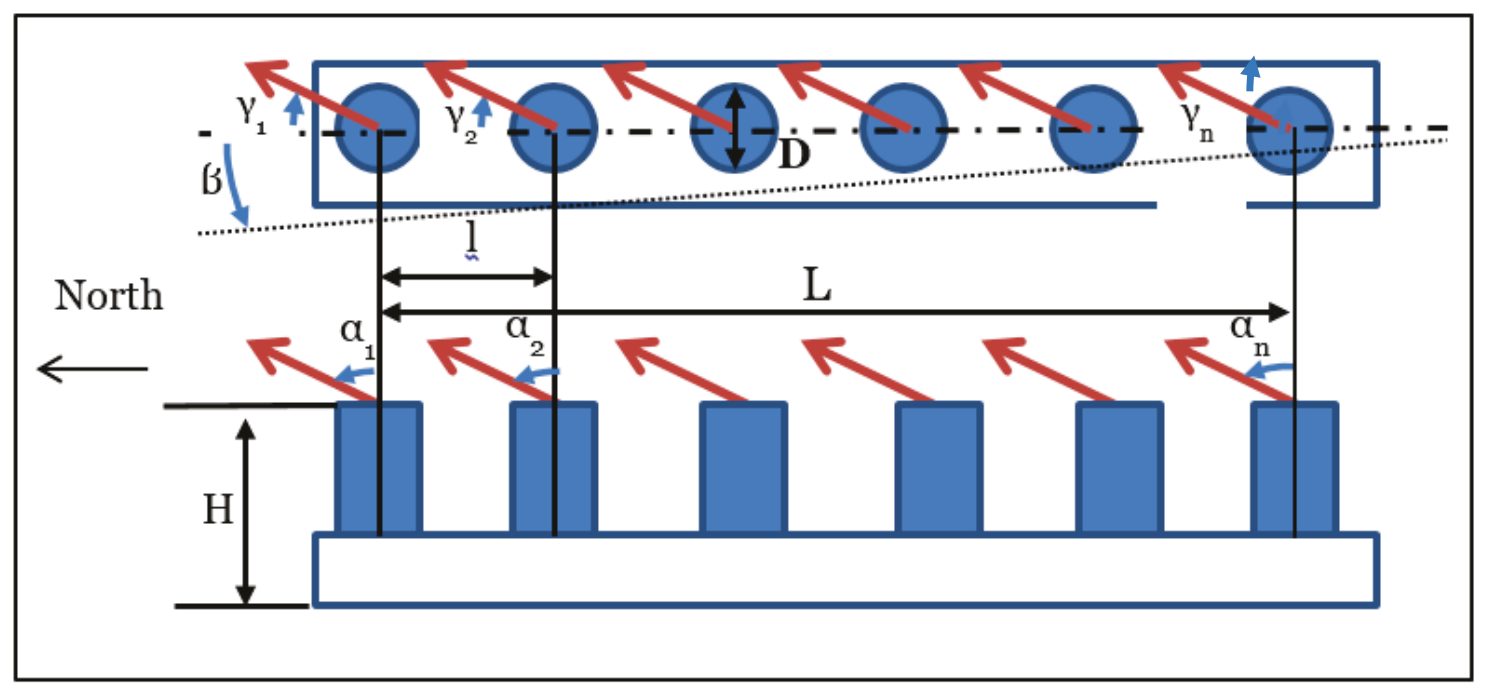




\section{Validation}

Previous physical model studies of the navigation traffic in the CSSC have not included an extensive validation effort. This stemmed from a lack of data collected from the field. A field campaign by the USGS and U.S. Fish and Wildlife Service provided enough data to conduct a validation for the return current generated by a passing vessel. This chapter will review the data collected by the USGS, the supporting data acquired from the AIS for vessel tracking, and the validation testing and results.

\subsection{Field data collected by USGS and channel flow}

On 31 August 2015, the USGS used a side-mounted Sontek Argonaut SW acoustic Doppler current profiler (ADCP) to measure velocities across the channel as the contracted vessel, the Lisa E., moved upstream and downstream in the CSSC. The ADCP was mounted on the west side of the channel at the electrical barrier at a $4.5 \mathrm{ft}$ depth and recorded data at $1.6 \mathrm{ft}$ bins, $2.5 \mathrm{ft}$ to $17.2 \mathrm{ft}$ cross channel from the wall. Velocity data were recorded in $1 \mathrm{~s}$ intervals in the downstream and cross-channel directions. Figure 4-1 shows the three inner bins ( $2.5 \mathrm{ft}$ to $5.74 \mathrm{ft})$ and three outer bin velocity averages (13.9 ft to $17.2 \mathrm{ft}$ ) for the time period of 12:54 to 14:05 CDT. The Lisa E. passed going downstream twice and upstream once in this time period. The data show that when the tow passed the barrier going downstream at 13:04 CDT, the velocity changed direction for approximately 2.5 minutes ( $\mathrm{min}$ ). After crossing the barrier, the tow changed sides of the barges and proceeded upstream increasing the downstream velocity to a recorded maximum of $1.5 \mathrm{ft} / \mathrm{s}$. Another downstream pass followed at 13:53 CDT. The ADCP shows significant change in velocity even 20 min after the passage of the barrier. Due to the possible contamination of the velocity from currents generated in the channel by the tow turning around, a decision was made to compare the laboratory validation testing to the first downstream passage that started at 13:04 CDT. Before the barge's passage, the average downstream velocity was approximately $0.75 \mathrm{ft} / \mathrm{s}$. The USGS also operates a stage and discharge gauging station in Lemont, IL (USGS 05536890 Chicago Sanitary and Ship Canal NR, Lemont, IL). The average discharge during this period was $2,900 \mathrm{cfs}$ at a $25.3 \mathrm{ft}$ depth with an average flow velocity of $0.7 \mathrm{ft} / \mathrm{s}$. These measurements are in good agreement with the stream velocity recorded in the initial portion of the USGS barge passage testing. Using a pump monitored by an ultrasonic flow meter, the prototype 2,900 cfs discharge was scaled to a model discharge of $2.54 \mathrm{cfs}$. 
Figure 4-1. The spatially averaged velocity for the three inner and three outer ADCP bins from 12:54 to 14:05 CDT.

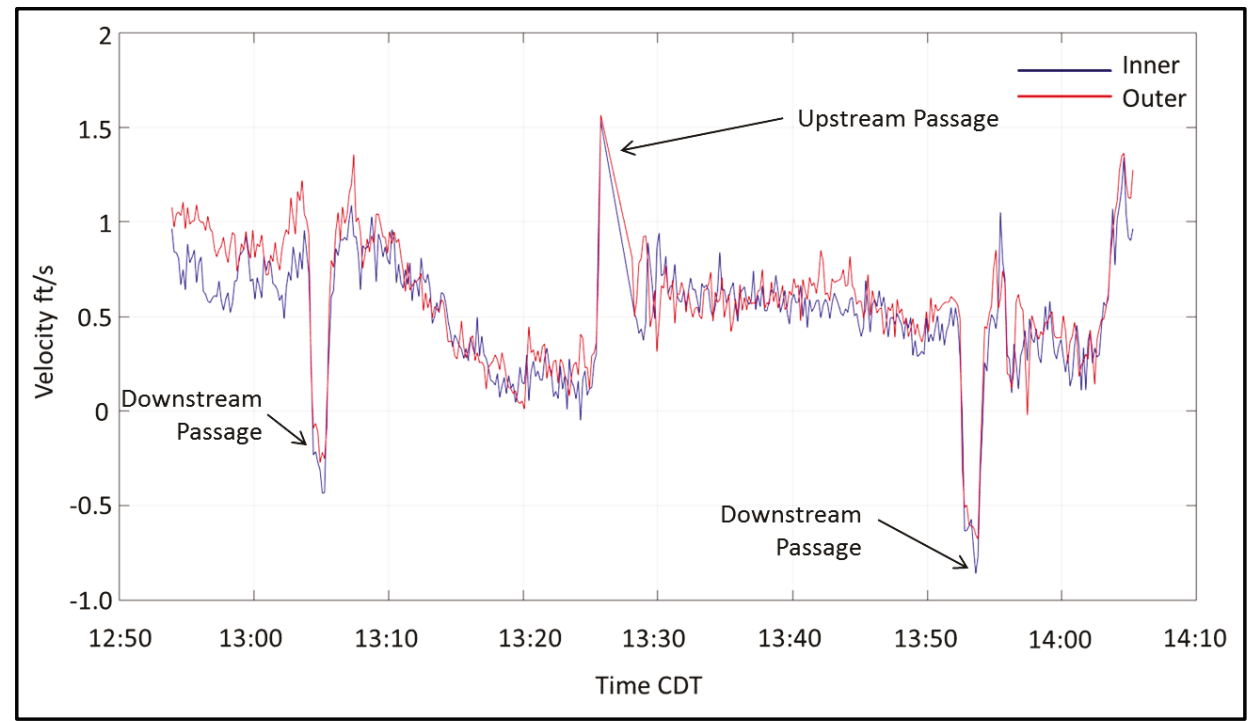

\subsection{Validation test parameters}

The model flow parameters were calculated by the velocity and discharge data taken from the ADCP and USGS Lemont, IL, gauging station. To obtain the correct return current, it was important to have the vessel speed, position, and barge configuration. The USGS provided the details of the barge configuration shown in Figure 4-2. For the southbound barrier crossing, the tow pushed a 2-wide by 2-long configuration with the front barges having box ends. The barges were $390 \mathrm{ft}$ long and $70 \mathrm{ft}$ wide and were fully loaded with a draft of $9 \mathrm{ft}$. This configuration was recreated for the laboratory testing.

Figure 4-2. Barge configuration the Lisa E. was pushing during the downstream return current testing on 31 August 2015.

\begin{tabular}{|l|lr|lr|}
\hline Lisa E. Tow & Box End & Box End & Box End & Box End \\
\hline & Rake End & Box End & Rake End & Box End \\
& \\
Direction of Travel \\
(Downstream)
\end{tabular}


High-resolution AIS data provide, in 1 min intervals, details on the position of vessels traveling along inland corridors. Using this data, the track of the Lisa E. as it passed through the barrier on 31 August 2015 from 12:48 to 13:10 CDT is presented in Figure 4-3. The vessel was stopped at the northern location before accelerating and passing through the barrier at 13:04 CDT. It stopped just south of the bridge in the image. Using the GPS coordinates from AIS, the vessel speed (over ground) was calculated. Figure 4-4 shows the vessel's speed as it passed through the barrier. The red line represents the time at which the Lisa E. passed the $\mathrm{ADCP}$. The average vessel speed (over ground) while passing the ADCP was estimated to be $3.4 \mathrm{mph}$. Since the barges extend $390 \mathrm{ft}$ in front of the tow, the return current began before the red line. The leading edge of the barges being pushed at $3.4 \mathrm{mph}$ in the configuration presented would pass the ADCP $78 \mathrm{~s}$ before the tow.

Figure 4-3. Path of Lisa E. on 31 August 2015 from 12:48 to 13:10 CDT taken from AIS data. Red pin shows the location of the USGS ADCP measurements.

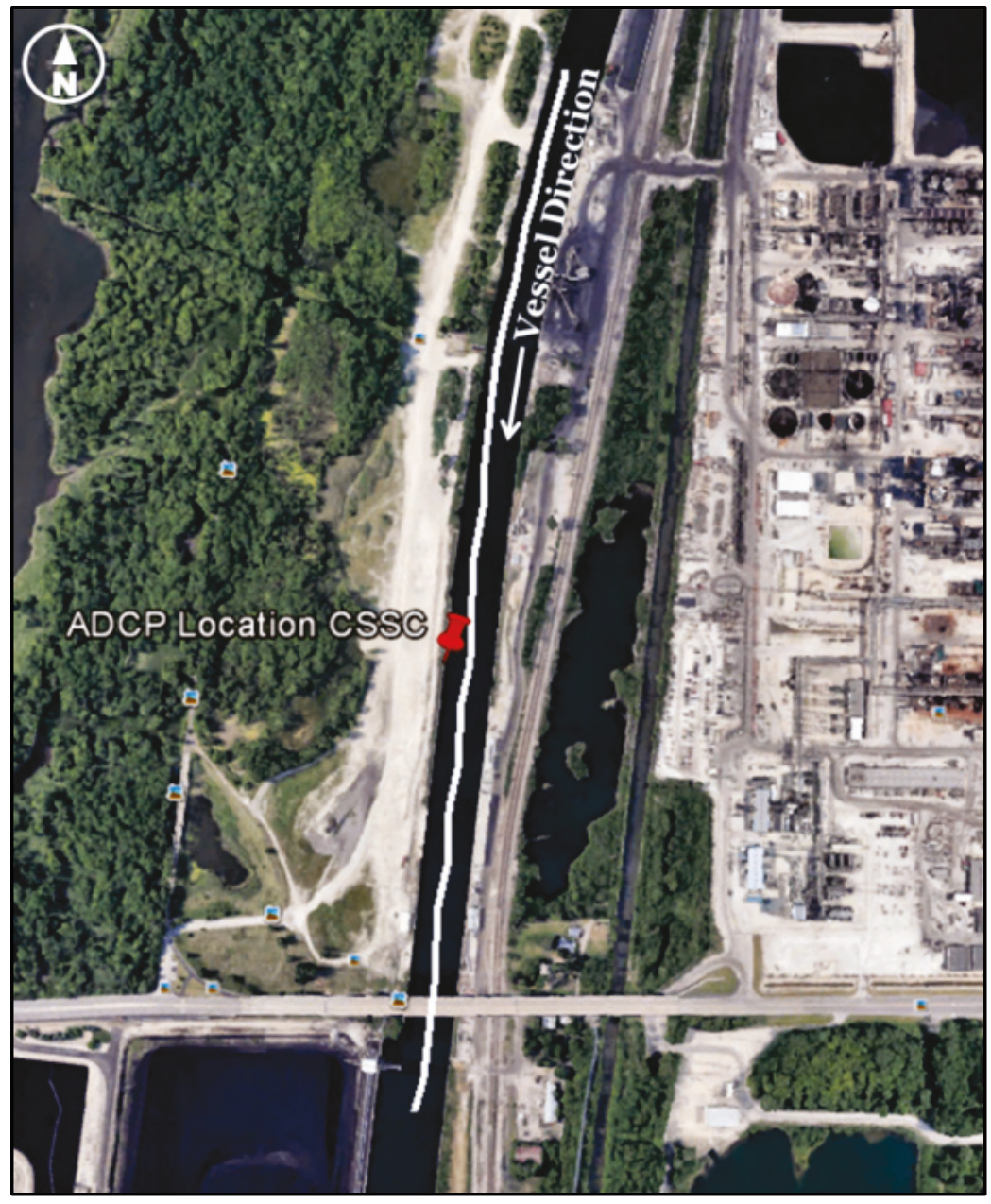


Using the presented information, the physical model could recreate the downstream passing of the ADCP. Since the ADCP instrument used in the field does not work well at the model scale, three Nortek Vectrino ADVs were used to measure the velocity before, during, and after the vessel passage in the laboratory. The instruments were placed at prototype positions of $4.1 \mathrm{ft}, 9.0 \mathrm{ft}$, and $13.9 \mathrm{ft}$ from the flume wall, sampling at a $4.1 \mathrm{ft}$ depth. These locations correspond with three sampling bins in the ADCP data. Figure 4-5 provides a picture of the ADV setup and the direction of channel flow.

Figure 4-4. The over-ground speed of the Lisa E. between 12:47 and 13:10 CDT, taken from the AIS data. Red line indicates the tow passage by the USGS ADCP.

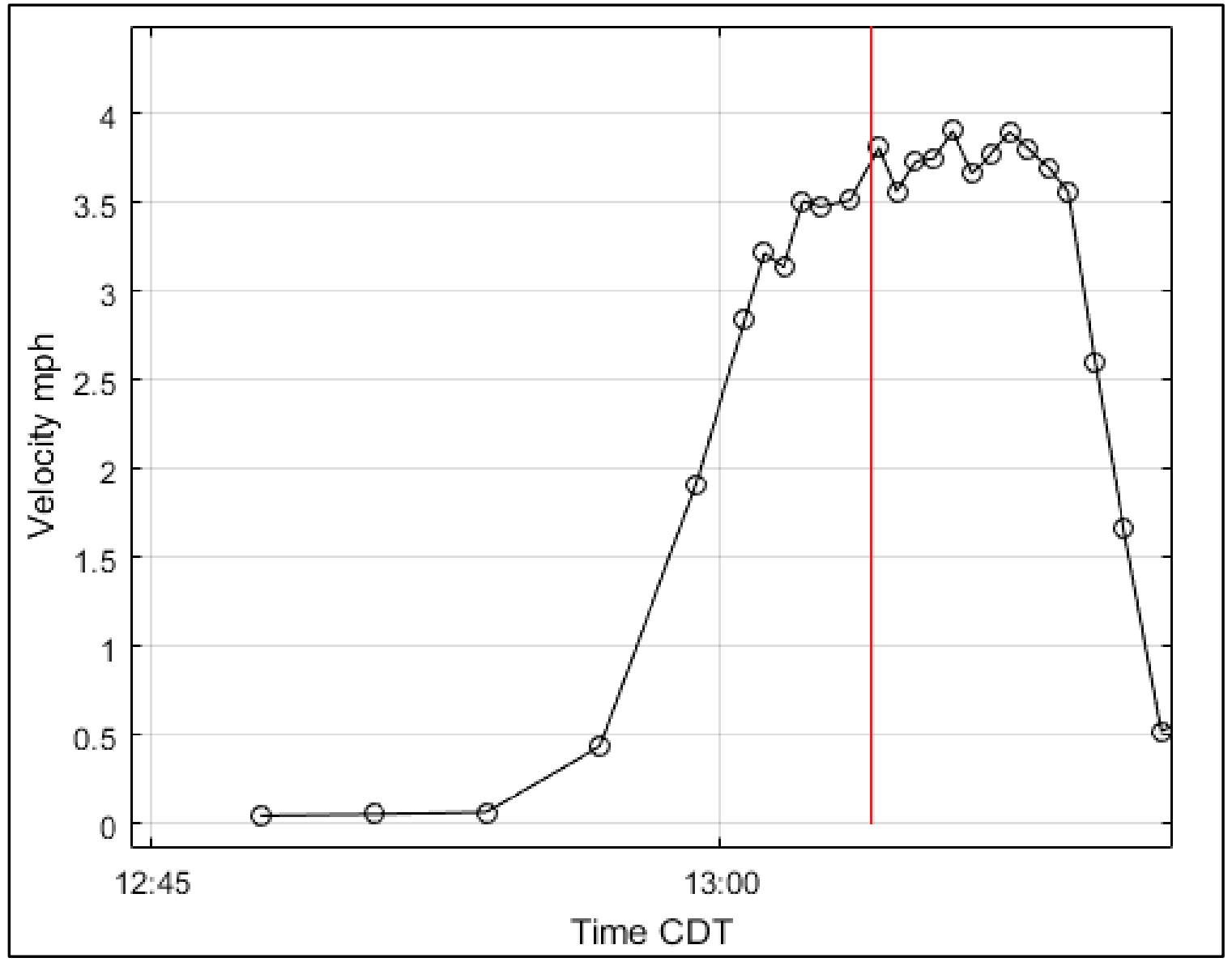


Figure 4-5. Picture of the channel setup with the three ADVs placed along the left wall. The direction of flow is also shown.

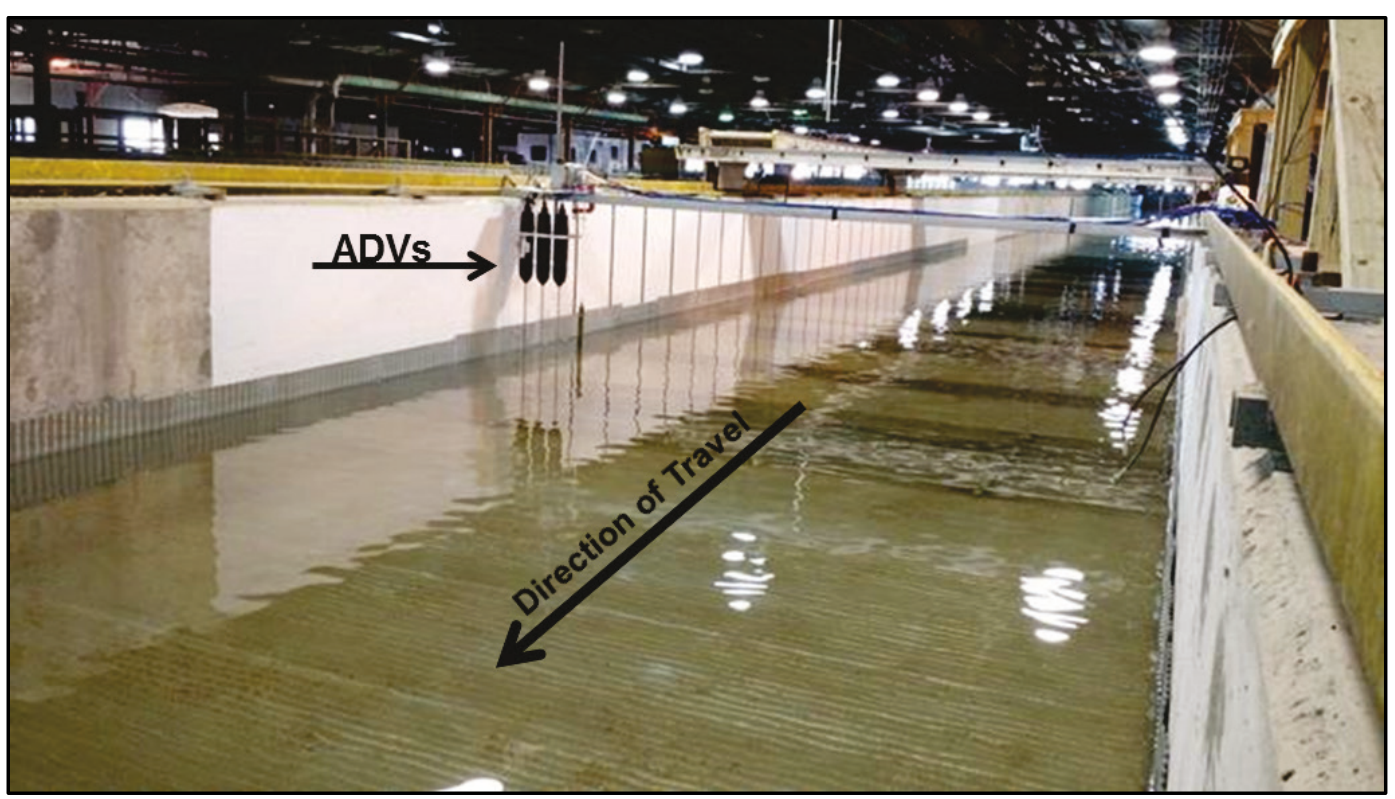

\subsection{Replication of results in physical models}

The parameters defined in the previous sections were used for the physical model testing. The test began with setting the model channel flow and verifying the velocity. Once the channel moved to an equilibrium state, the ADVs began sampling the velocity. The tow would then begin at the far end of the channel and pass the ADVs. The vessel speed was calculated by measuring the time of passage over a known length. After vessel passage, the ADV data were analyzed and compared to the ADCP data. ADVs record point velocities and sample at a much higher rate than the ADCP used in the field. For the testing, the ADVs sampled at $25 \mathrm{~Hz}$. However, for the following results, the data are averaged over and presented at $1 \mathrm{~Hz}$ prototype and compared to the ADCP bins $4.1 \mathrm{ft}, 9.0 \mathrm{ft}$, and $13.9 \mathrm{ft}$ from the canal wall. Figure 4-6 shows a comparison between velocity in the model and prototype for an over-ground vessel speed of $3.39 \mathrm{mph}$. The channel velocity was well matched before the vessel arrived in the first $10 \mathrm{~min}$. The shape and magnitude of the return velocity is also well matched. The time of the return current was 20 to $30 \mathrm{~s}$ shorter in the model than the prototype. This difference could be caused by the model vessel velocity and path being slightly different from the prototype velocity. 
Figure 4-6. Velocity comparison between model and prototype for the validation testing with a tow velocity of $3.39 \mathrm{mph}$.

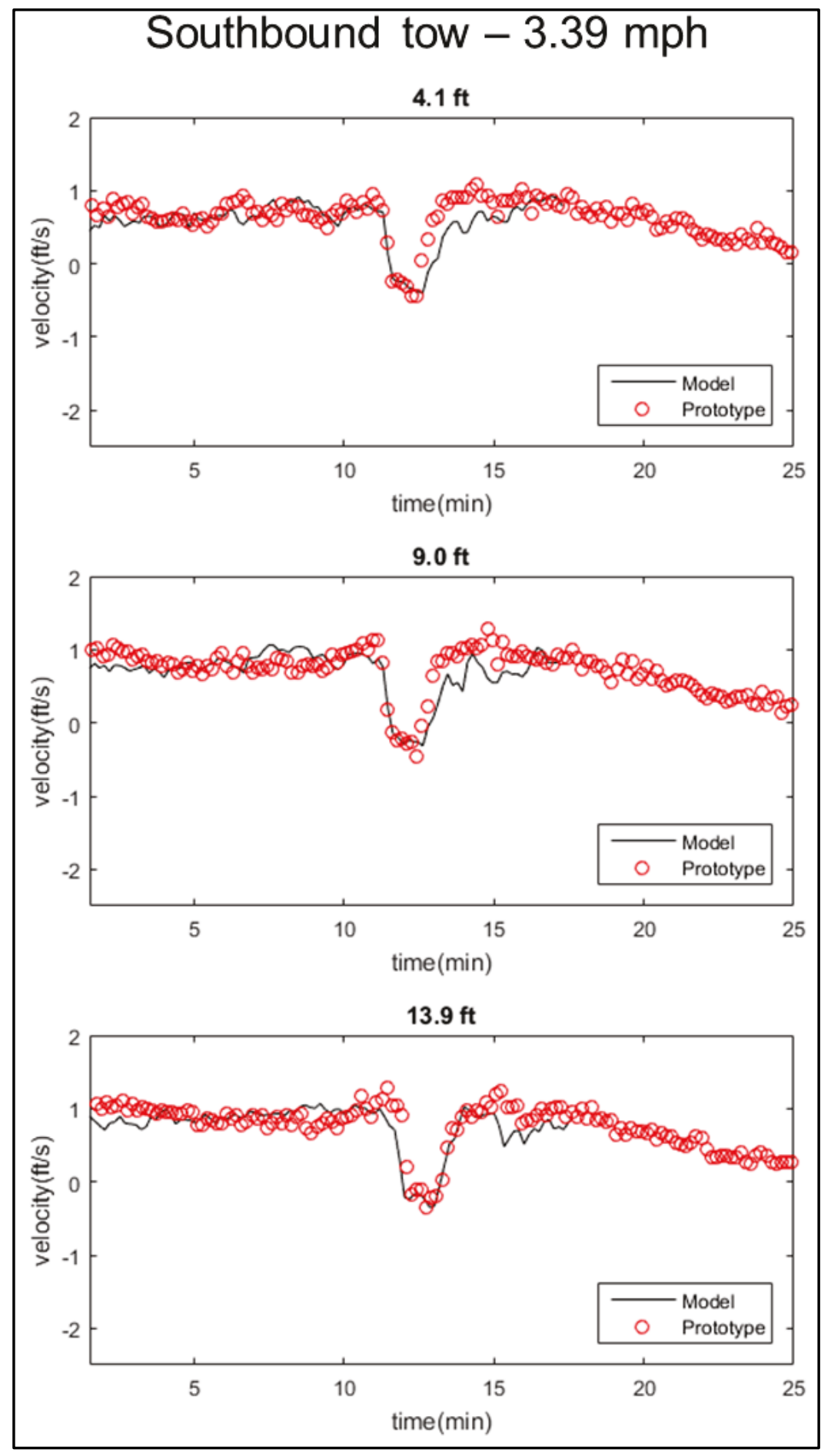


Figure 4-7 shows the return current velocity comparison for a model vessel velocity of $3.41 \mathrm{mph}$. As with the previous comparison, the canal velocity was well matched as was the shape and magnitude of the return velocity. These results show that the model recreates the hydrodynamics in the canal during vessel passage well.

Figure 4-7. Velocity comparison between model and prototype for the validation testing with a tow velocity of $3.41 \mathrm{mph}$.

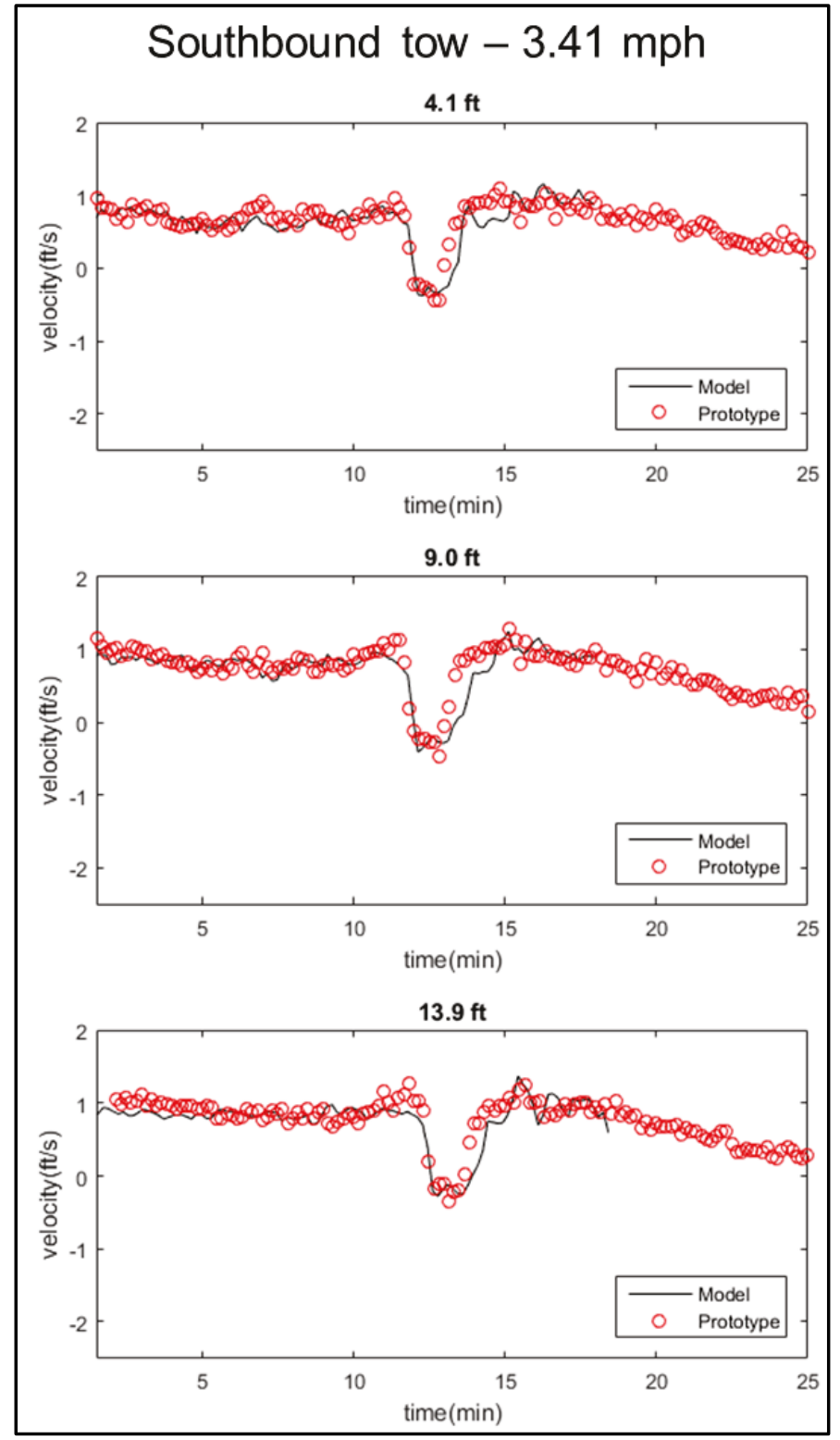




\section{Return Current Passage}

This chapter is focused on the entrainment and transport of fish into the return current. The vessel-generated return current was identified by Bryant et al. (2016) as a plausible method for fish transport through the CSSC electric barriers as its generation is consistent and acts over a very large cross section of the flume. The results presented here will help to clarify the size of that action area, the flow velocities, the time of impact and the distance an object could be moved by the return current. All results are presented in prototype scale.

\subsection{Specific methods for return currents}

Similarly to the validation testing, three ADVs were used to measure the return current generated under different speeds and configurations.

Figure 5-1 diagrams the two depths and six lateral distances from the wall at which the return current was measured. The first three lateral locations correspond with bins associated with the USGS ADCP data, 4.1, 9.0, and $13.9 \mathrm{ft}$. The farthest three lateral positions were set to $18.3,23$, and $28 \mathrm{ft}$ from the wall. Two water depths were also chosen. The first flow depth corresponds to the sampling depth of the USGS ADCP at $4.1 \mathrm{ft}$, and the second flow depth corresponds to half a typical flow depth at the barrier, $13.1 \mathrm{ft}$. Since only three ADVs were available for use, the tests were rerun at least three times for each ADV lateral and depth location. From that data, the mean return current velocity, the mean length of the return current, and the mean displacement distance were calculated. These three values are the metrics used for differing vessel speeds and configurations.

Figure 5-1. Depths of return current velocity measurements.

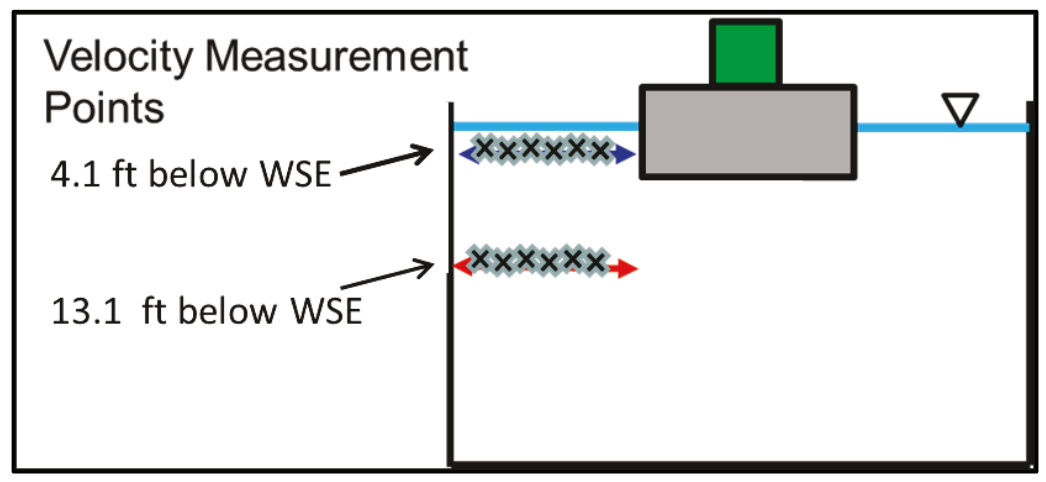




\subsection{Testing procedure}

All testing for the return current was performed without a current in the canal except for a series of tests used to demonstrate the impact of channel flow. That result will be shown at the end of this chapter. Before the vessel began to move, the ADVs were turned on and set to sample at $25 \mathrm{~Hz}$. The vessel then accelerated to a speed of either 2.4 or $3.5 \mathrm{mph}$. The vessel tracked along the center of the channel and passed the ADVs completely. Once the vessel had passed and the flow became quiescent again, the ADV sampling would end. A time of 20 min passed between tests to allow any residual currents to dissipate. Six configurations with raked front barges were tested. These configurations are a single barge, a 1-wide by 2-long, a 1 -wide by 3 -long, a 2 -wide by 1 -long, a 2 -wide by 2 -long and a 2 -wide by 3 -long. The 2-wide by 3 -long tow configuration is the largest that passes through the barrier.

\subsection{Results}

The lateral and vertical distributions of the return current metrics were first measured at the 12 locations identified in the previous section. These measurements were taken for a 2-wide by 3-long tow traveling at $2.4 \mathrm{mph}$ and $3.5 \mathrm{mph}$. Figure 5-2 shows the observed time of the return current. A tow traveling at $2.4 \mathrm{mph}$ had a mean observed time between 4.5 and 5.25 min depending on the location whereas the tow traveling at $3.5 \mathrm{mph}$ had a mean observed time between 3.0 and 4.3 minutes. For both tows traveling at 2.4 and $3.5 \mathrm{mph}$, no noticeable pattern was found for the difference between the two measurements depths. Laterally, the mean times were similar. 
Figure 5-2. Observed time of return current at six lateral positions and two depths with error bars showing standard error of the mean for a 2-wide by 3-long tow.

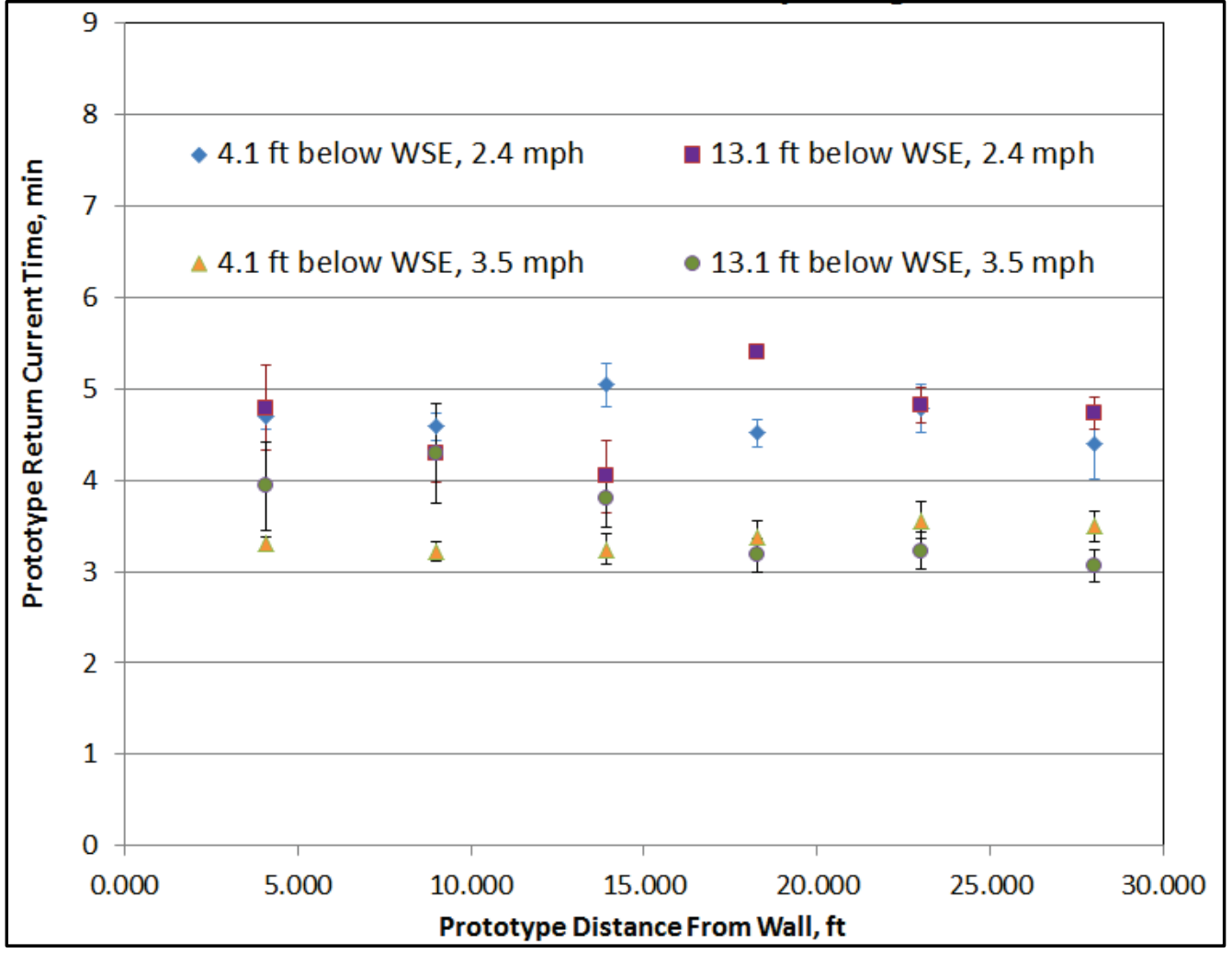

The measured mean return current was also fairly uniform with depth. Figure 5-3 gives the mean of the return current. A tow traveling at $2.4 \mathrm{mph}$ had a mean return current between 0.55 and $0.64 \mathrm{ft} / \mathrm{s}$ depending on the location whereas the tow traveling at $3.5 \mathrm{mph}$ had a mean return current between 0.67 and $0.82 \mathrm{ft} / \mathrm{s}$. For both tows traveling at 2.4 and $3.5 \mathrm{mph}$, no observable pattern was observed for the difference between the two measurements depths. As was the case with the observed length of the return current, the mean return current velocities were similar. Based on this data, it was concluded that the return current acts uniformly over the measured. 
Figure 5-3. Mean of return current (feet/second) at six lateral positions and two depths with error bars showing standard error of the mean for a 2-wide by 3-long tow.

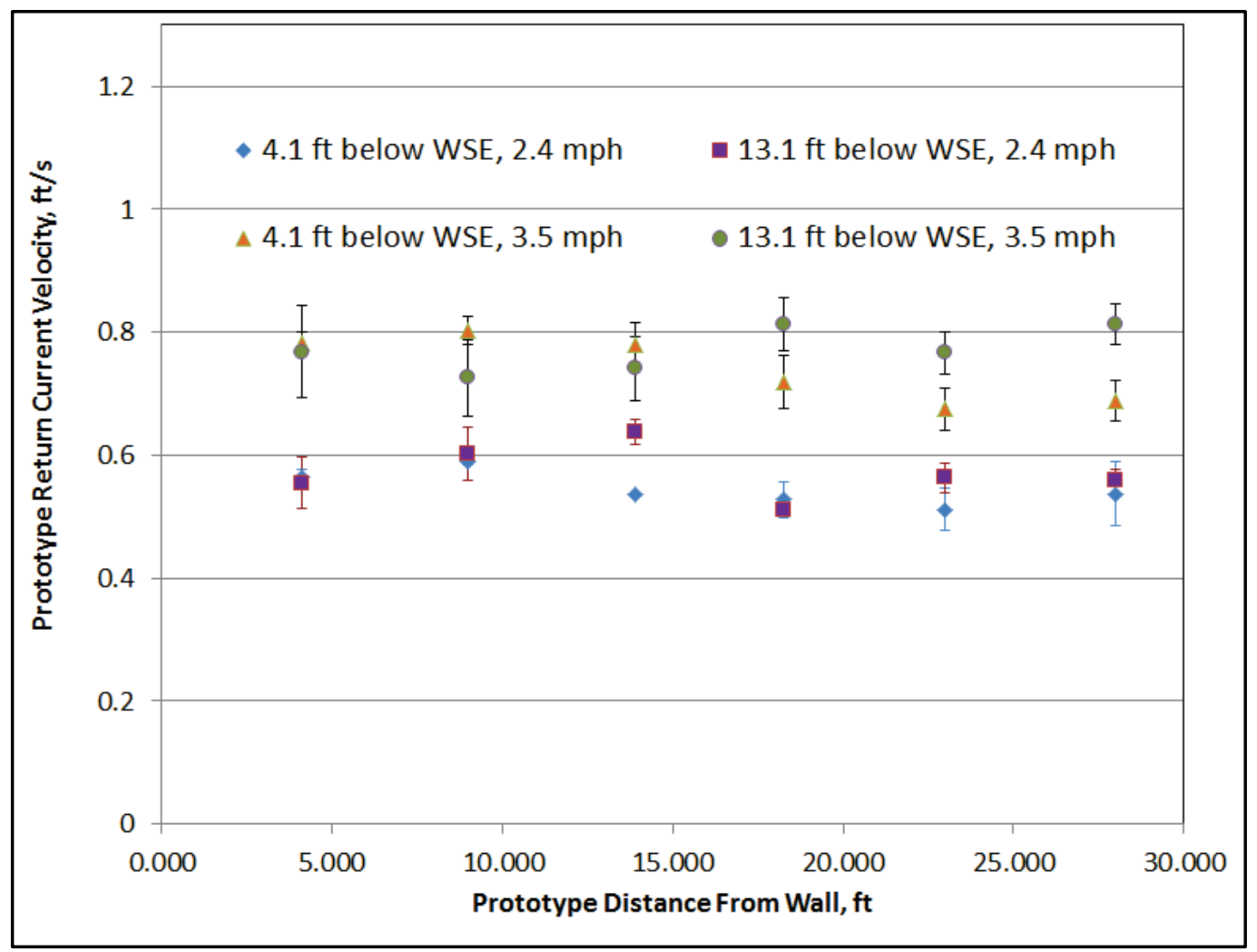

The recorded velocity can be integrated over the length of time the return current is acting to estimate the distance an object would travel. The mean travel distance or displacement distance is given in Figure 5-4. This distance is similar for both the $2.4 \mathrm{mph}$ and $3.5 \mathrm{mph}$ tow with a minimum of $140 \mathrm{ft}$ and a maximum of $183 \mathrm{ft}$. The $3.5 \mathrm{mph}$ tow averaged higher displacement distances near the canal wall, but the $2.4 \mathrm{mph}$ tow did not. 
Figure 5-4. Calculated average displacement distances (feet) at six lateral positions and two depths with error bars showing standard error of the mean for a 2-wide by 3-long tow.

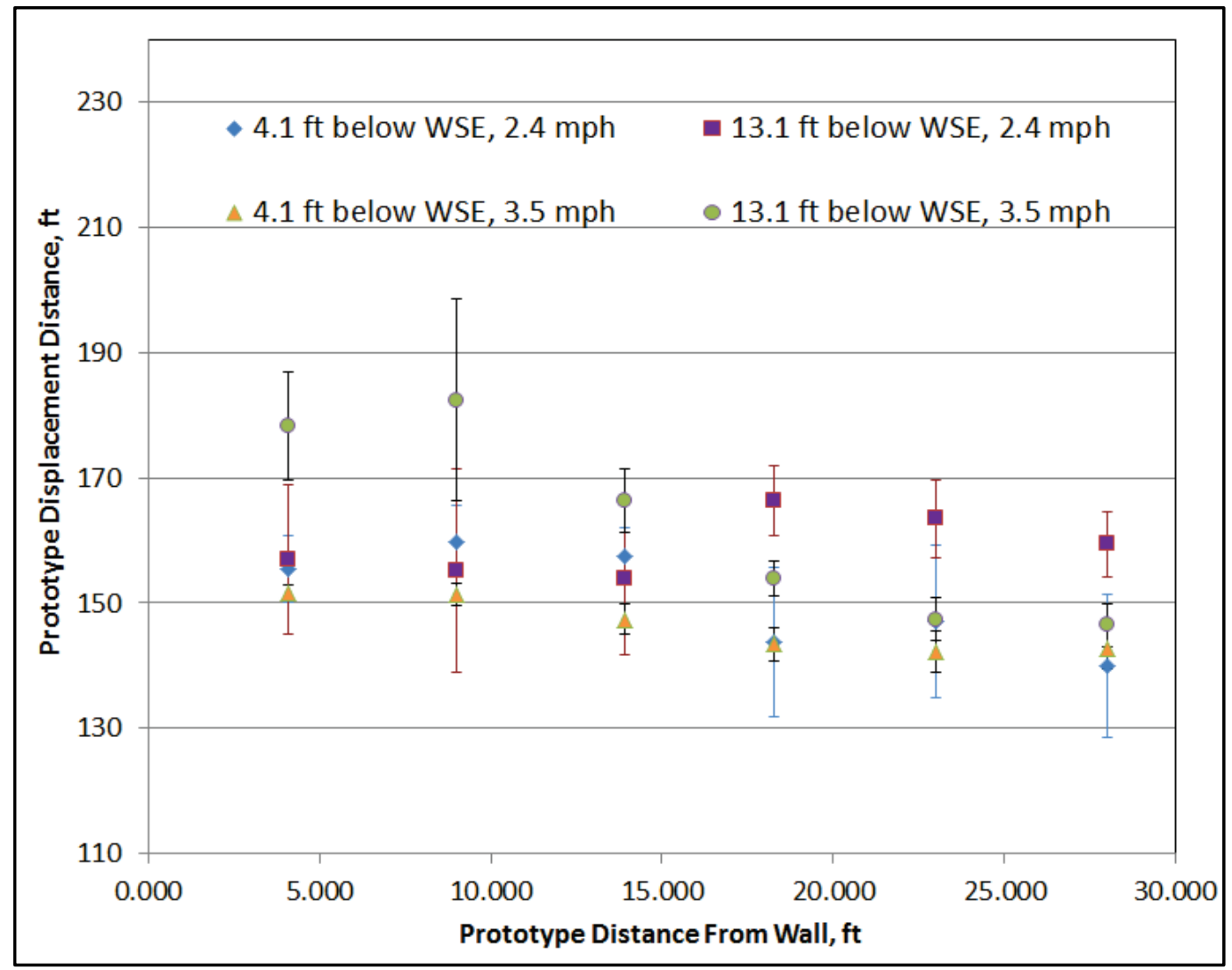

With the return velocity being similar from the canal wall to $28 \mathrm{ft}$ laterally, data to compare return current due to different configurations were only measured for the positions closest to the wall. Figure 5-5 shows the mean observed time of the return for $2.4 \mathrm{mph}$ and $3.5 \mathrm{mph}$ for all configurations tested. The standard error of the mean is also calculated and presented with error bars. An increase in vessel speed reduces the duration of the return current for all configurations. Both the tow width and length effect the duration of the return current. For the 1-wide tow, an additional barge length increased the duration of the return current by approximately $1 \mathrm{~min}$. A 2-wide by 1 -long tow had an approximately 1 min increase in return current duration over a 1-wide by 1-long tow. The 2-wide by 2-long and 2-wide by 3 -long tows had similar durations for both the $2.4 \mathrm{mph}$ and $3.5 \mathrm{mph}$ vessel speeds. Knowing the duration of the return current can aid in designing counter measures. However, the flow velocity must also be considered. 
Figure 5-5. Mean observed time (minute) of return current for six barge configurations and two vessel speeds with calculated standard error.

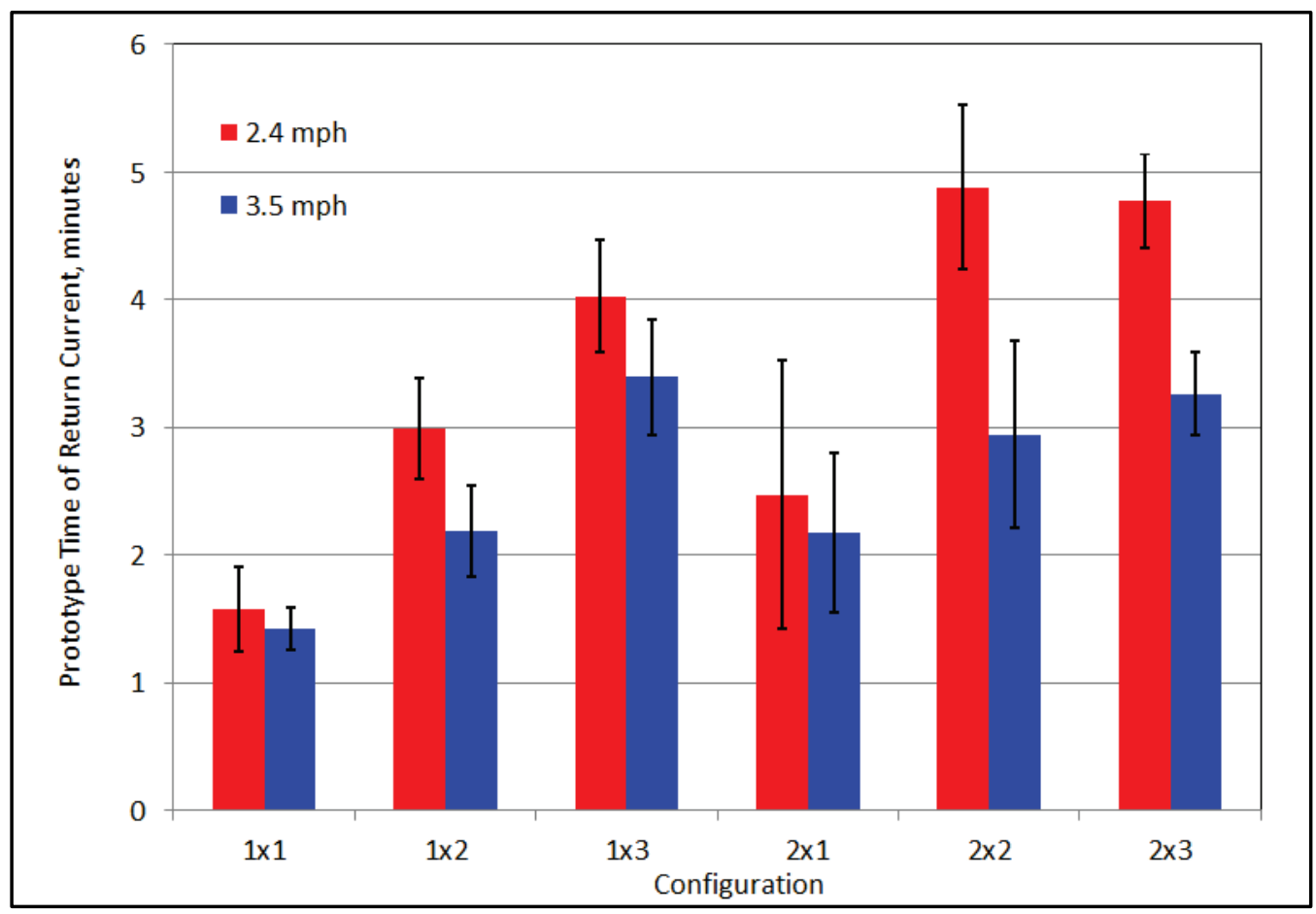

The mean velocity of the return current is given in Figure 5-6 for all configurations. The longer tow configurations caused an increased return velocity as did a wider tow configuration. These findings indicate that the mean velocity of the return current is a function of both the projected frontal area and total volume displaced. The minimum mean velocity of just under $0.2 \mathrm{ft} / \mathrm{s}$ was measured for the 1-wide by 1-long configuration traveling at $2.4 \mathrm{mph}$. Increasing the vessel speed to $3.5 \mathrm{mph}$ increased the mean return velocity by approximately $25 \%$ for the 1 -wide by 1 -long tow configuration and approximately $43 \%$ for the 2-wide by 3 -long tow configuration. The largest mean return velocity was $0.78 \mathrm{ft} / \mathrm{s}$ for the 2-wide by 3-long configuration traveling at $3.5 \mathrm{mph}$. As shown previously, the return velocity can be integrated over the duration time to determine a total displacement distance. Average higher return current velocities will result in larger displacement distances. Thus, the mean return currents measured can be used to improve the design of methods to negate risk from return current entrainment. 
Figure 5-6. Mean velcoity ( $\mathrm{ft} / \mathrm{s})$ of return current for six barge configurations and two vessel speeds with calculated standard error.

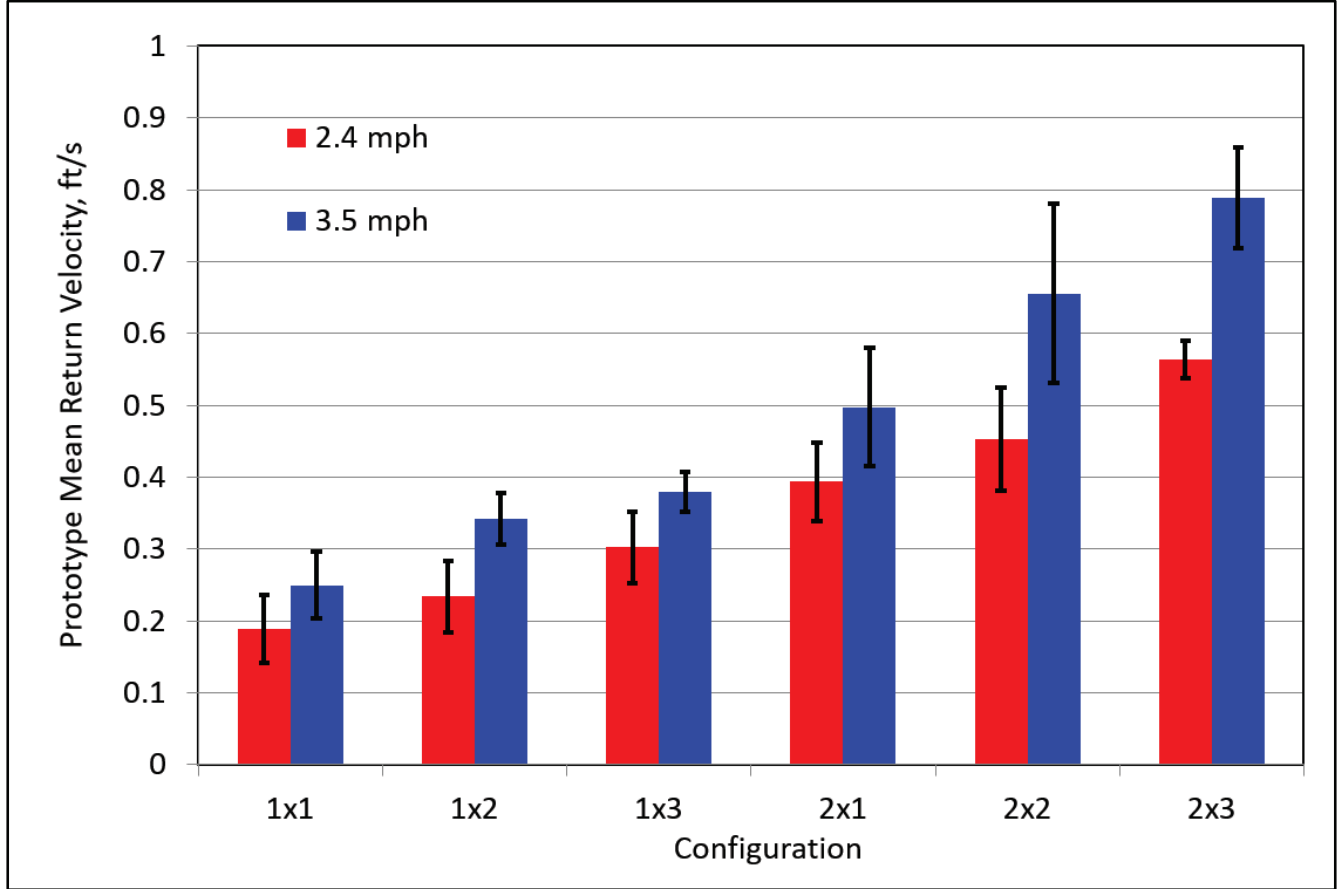

Using the measured velocities, the displacement distance was found for each configuration for tows traveling at 2.4 and $3.5 \mathrm{mph}$. The mean current displacement distance is provided in Figure 5-7. Additionally, a 98\% confidence interval was also calculated for the measurement. Depending on the configuration and vessel speed, the mean displacement distance measured from 15 to $138 \mathrm{ft}$. In four of the six configurations tested, a vessel speed increase also led to a displacement distance increase. The 2-wide by 2-long and 2-wide by 3-long configurations produce slightly smaller mean displacement distances with increasing vessel speed. The 98\% confidence interval is much smaller for the smaller tow configurations than for the larger tow configurations. However, the trend is clear: a larger tow will produce a larger displacement distance. 
Figure 5-7. Mean calculated return current displacement distances with the $98 \%$ confidence interval.

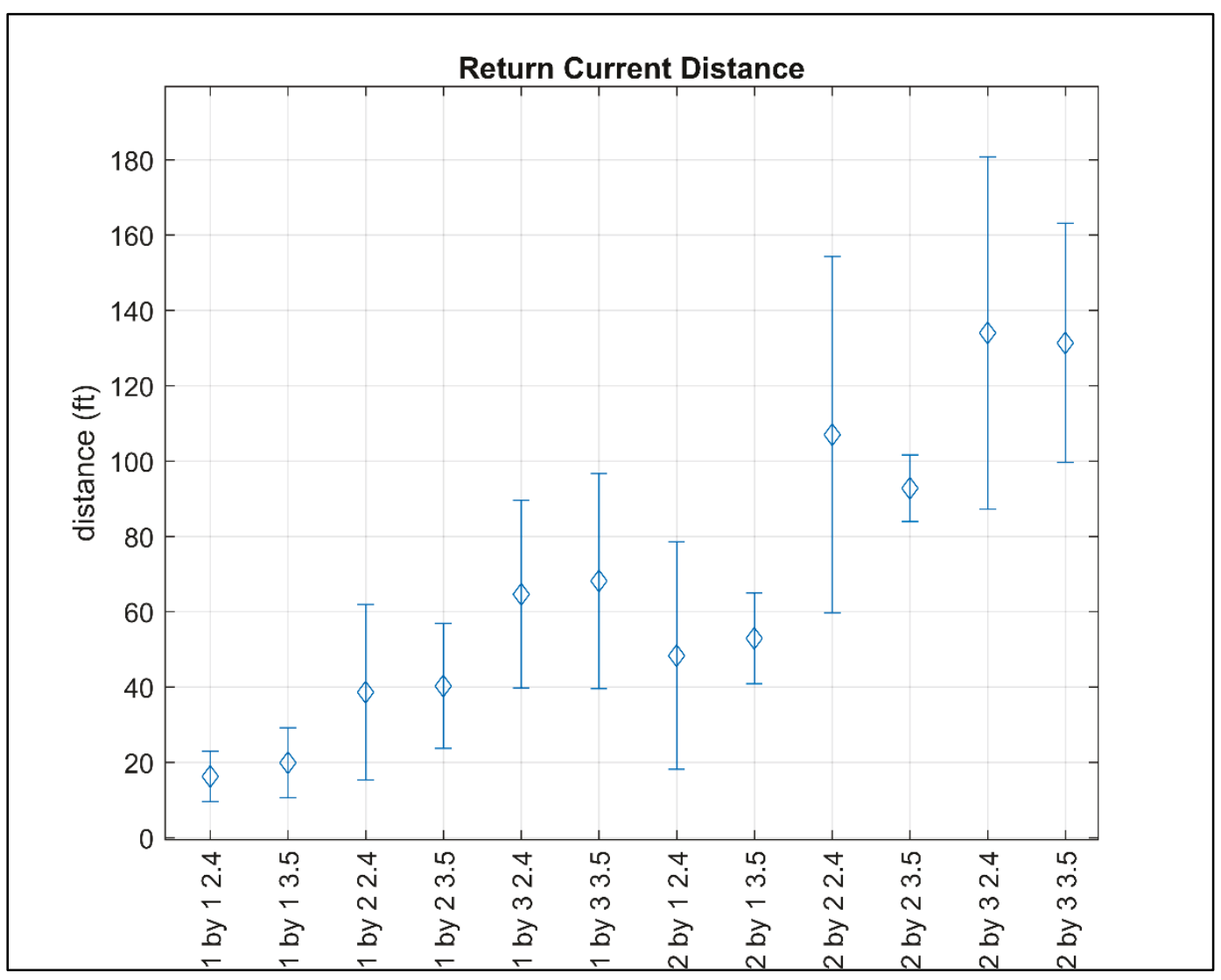

For future design purposes, the $99 \%$ one-tailed confidence interval is presented in Figure 5-8 in addition to the maximum measured current displacement distances. These data reinforce the dependence of displacement distance on configuration. For a 2-wide by 2-long tow traveling at $2.4 \mathrm{mph}, 99 \%$ of the displacement distance will be $130 \mathrm{ft}$ or less. The 2-wide by 3-long configuration with a vessel speed of $3.5 \mathrm{mph}$ had the highest $99 \%$ one-tailed confidence interval of $191 \mathrm{ft}$. Tow configuration of 1 -wide by 1 -long and 1-wide by 2 -long had the smallest displacement distances. Figure 5-8 demonstrates that limiting the number of barges can reduce risk of the return current transporting fish. 
Figure 5-8. Maximum measured return current displacements (feet) with the $99 \%$ one-tailed confidence interval maximum displacement distances.

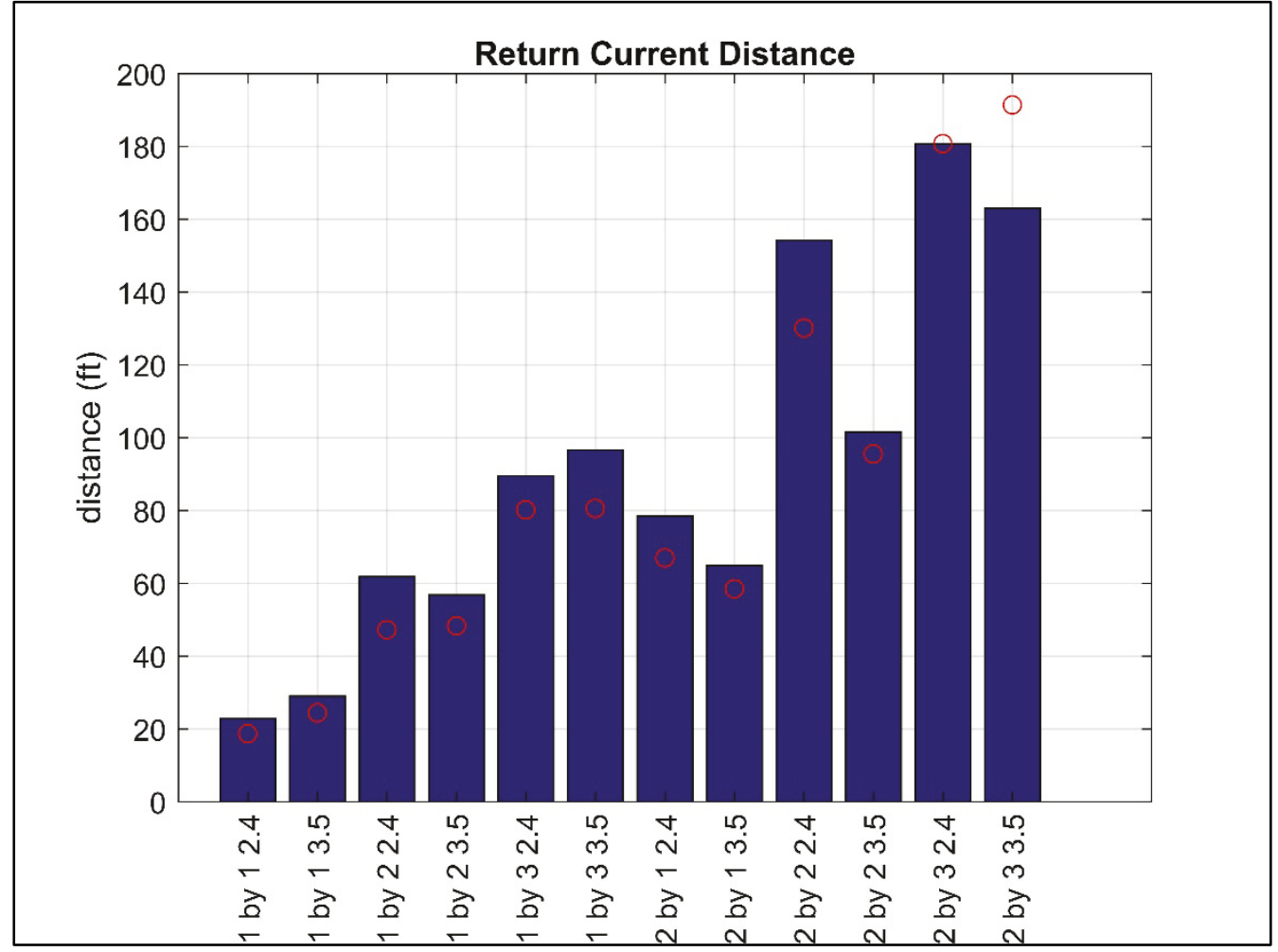

Beyond vessel speed and tow configuration, another important factor in the displacement distance is the flow in the canal. As observed in the collected USGS field data, the return current can be offset by a flow in the canal. Figure 5-9 shows how a canal flow could reduce or eliminate the risk of the return current for a 2-wide by 2-long barge. At the three locations (4.1, 9.0, and $13.9 \mathrm{ft}$ ), the peak return current velocity is approximately $0.65 \mathrm{ft} / \mathrm{s}$ upstream (negative values) for a quiescent flow situation. However, increasing the mean canal velocity to $0.8 \mathrm{ft} / \mathrm{s}$ eliminates the flow moving upstream. A further increase in canal velocity to $1.3 \mathrm{ft} / \mathrm{s}$ has a greater effect on the return current providing additional assurance against upstream displacement. While maintaining a constant flow rate and velocity in the canal may be unreasonable, using these data and the discharge data from the USGS Lemont station, detailed in Chapter 2, can provide a decision-based tool on when the risk of return current displacement through the barrier is high and when it is low. The ability to make real-time assessments on the potential risk could provide a greater operational efficiency to any countermeasures. 
Figure 5-9. Channel flow effect on return current for a vessel over water speed of $1.71 \mathrm{mph}$ at $4.1,9.0$, and $13.9 \mathrm{ft}$ from the canal wall.

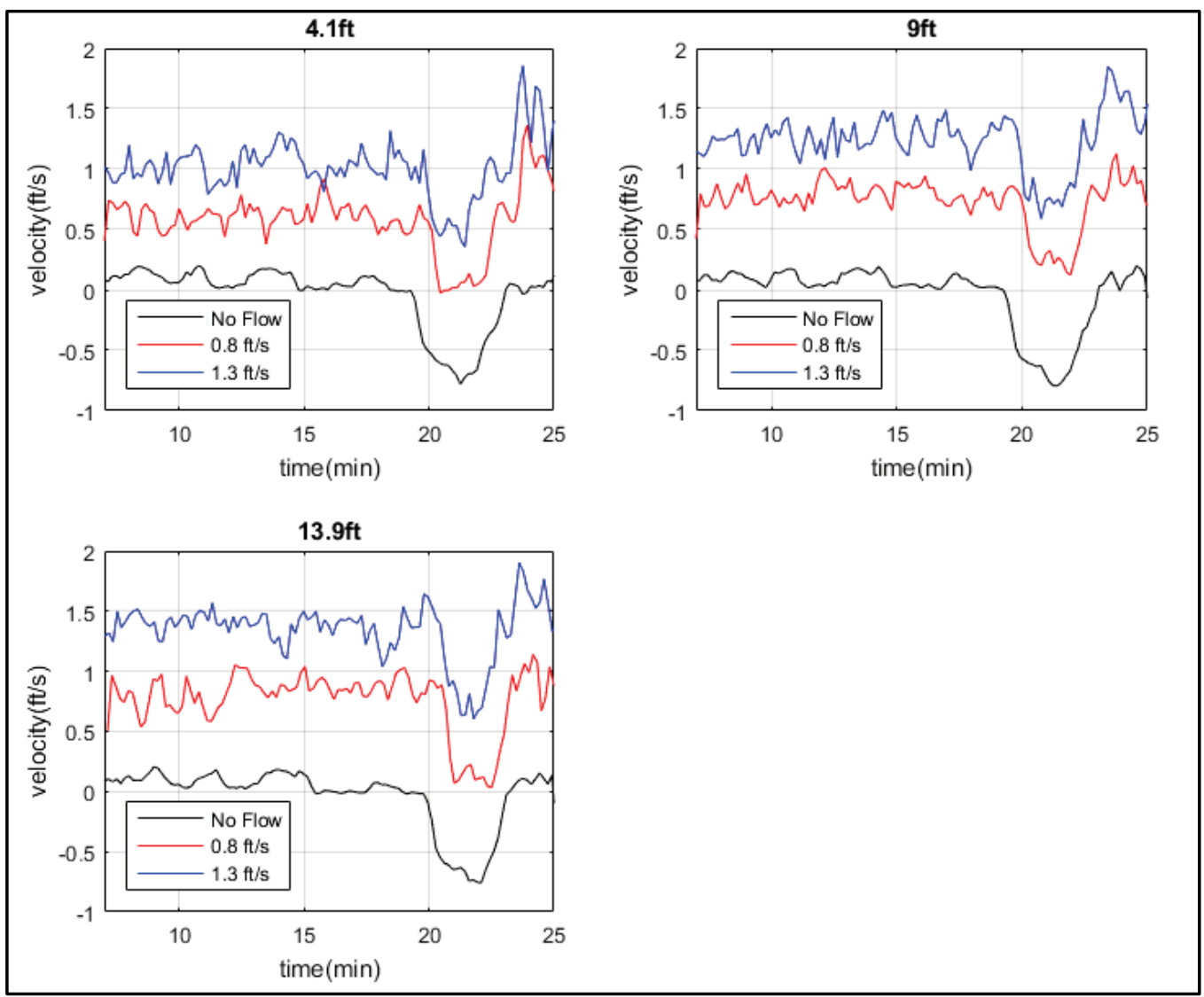

\subsection{Conclusion}

In this chapter the collection, analysis and results of the return current entrainment in the CSSC for different vessel speeds and configurations has been described in detail. The data show that larger vessels greatly increase the distance that a fish could be carried through the barrier. A relationship between vessel speed and displacement distance was unclear. For smaller tow configurations, a greater speed increased the displacement distance, but for larger tows the opposite occurred, though the measured differences were small. The data are for tows traveling down the center of the CSSC, but the cross-canal position could increase the return velocity greatly to one side. All the tow configurations tested had leading raked barges.

The previous study initiated in 2011 (Bryant et al. 2016) clearly showed that a box barge leading the tow configuration produces greater displacement distances. This condition was not tested again in this study because the use of box barge leading tow configurations is avoided by the tow industry due to increased fuel consumption. Additionally, this analysis 
does not take into account any fish behavior, as that was outside the scope of this study. The data shown here, when properly combined with current conditions in the canal, tow configuration, and vessel speed, can be used to make a real-time assessment on the risk and take the necessary action. A net upstream return current can only be eliminated by creating a flow in the canal that is either locally produced at the barrier or by controlling the canal discharge itself. Last, changes to the canal could be used to force the fish that may be moved by the return current down into a high-current region where they would not survive the passage. 


\section{Entrainment and Flushing of Barge Junction Recesses}

\subsection{Testing procedure}

Before any flushing tests, the model tow was calibrated for a given tow configuration and water depth to establish the relationship between propeller speed and tow speed relative to water. The model towboat was equipped with a tachometer that measures the rotational speed of the propellers.

In the previous 2011 study, the void space due to the junction of a box end barge and a raked front barge was shown in the previous study to present the potential for long distance transport for northbound traffic. Two primary solutions were presented to remove fish from this region: barge maneuvers and jet arrays. Each solution was initially tested, and specific configurations and methods that showed promise early in testing were tested more extensively by applying additional variables that influence entrainment.

\subsubsection{Maneuvers}

The first maneuver was a swing-out maneuver that included a variation of performing it with and without a tie-off. To perform this maneuver, the barge and tow were initially at rest with the starboard side along the channel wall. First, using dual motors, the front of the barge was moved from the channel wall with the port side first. For the tie-off, a member of the team secured the back starboard side of the barges to keep this corner in contact with the channel wall throughout the swing-out maneuver. The barge was then aligned with the channel centerline and traveled a short distance (less than 10 barge lengths) down the model channel. Model fish were placed into the rake area while the barge was at rest before the maneuver began.

The second maneuver is referred to as a hard stop and reverse. At the start of this maneuver, the barge is traveling down the channel. Then, the barge comes to a complete stop. Finally, the barge is reversed for a distance until all model fish are cleared from the rake area. To evaluate the effectiveness of this maneuver, model fish were placed into the rake area as the barge approached the testing section before the hard stop and reverse was 
initiated. All maneuvering tests were recorded with both Go-Pro cameras and overhead cameras so speed and barge movement information could be collected following the testing. Figure 6-1 shows a sample data testing sheet used to record the results for maneuver testing.

Figure 6-1. Sample data sheet for maneuver flushing tests.

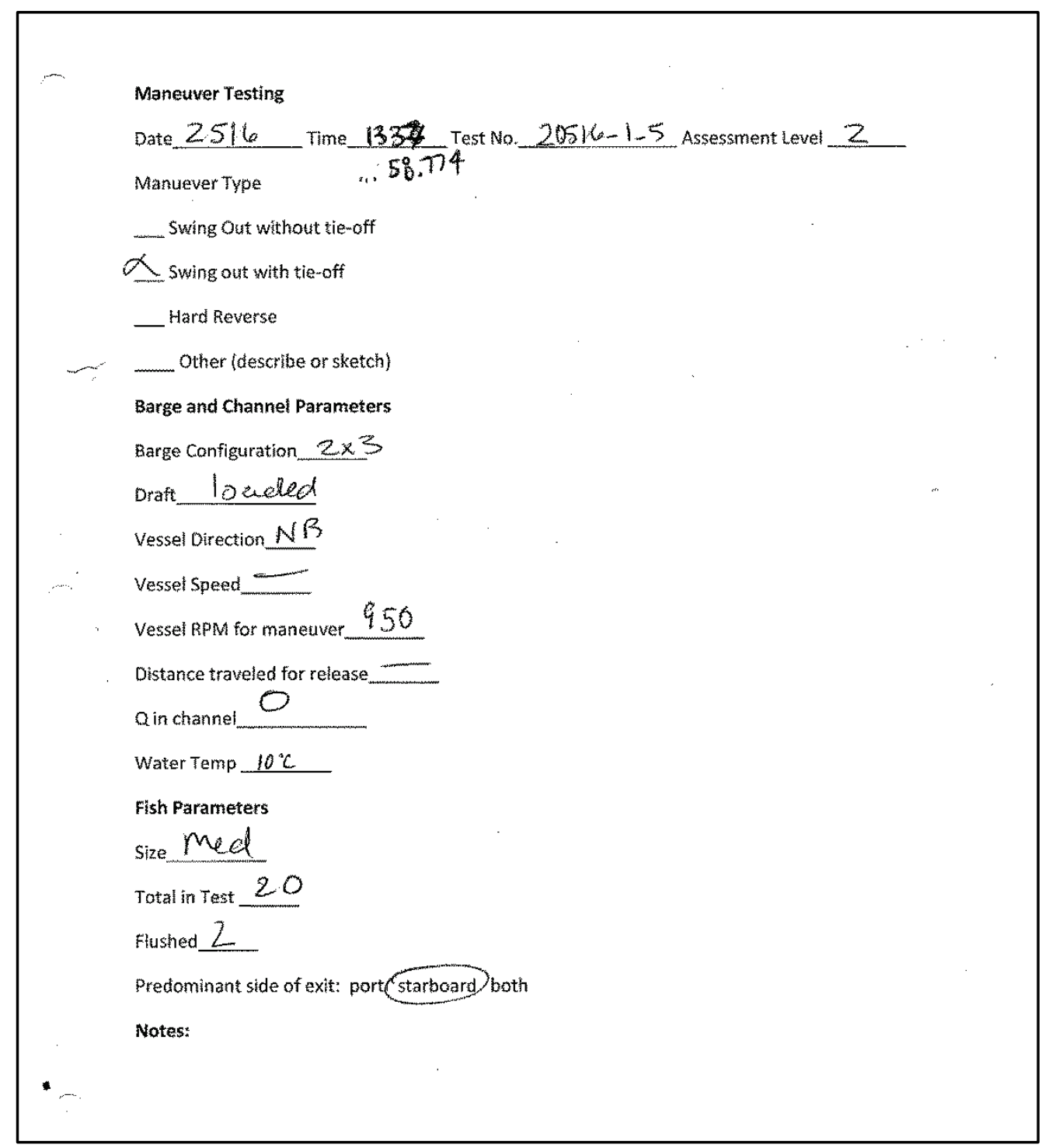

\subsubsection{Vertical jet tests}

Initially, jets were tested with no vertical angle $\left(\alpha=0^{\circ}\right)$. This configuration was found to be ineffective at clearing fish from the void area; however, this procedure worked well to clear fish from the channel centerline. Therefore, this jet configuration could be a viable way to clear fish from the path of an approaching barge. 
A rectangular jet array with 16.7 in. prototype diameter vertical jets was placed along the bottom center of the flume in the CHL facility. For these tests, two jet configurations were tested. The first configuration consisted of a $133 \mathrm{ft}$ prototype array with spacing of $133 \mathrm{in}$. on center. The second configuration incorporated a $67 \mathrm{ft}$ prototype array with jet spacing 33.4 in. on center.

The model jet array was connected to a cylindrical reservoir with a 5 -horsepower (hp) centrifugal pump by 2 in. diameter pipes. The flow rate to the jets was measured using an Endress Hauser electromagnetic flow meter. Three different prototype flow rates were tested during the trials: $130 \mathrm{cfs}, 250 \mathrm{cfs}$, and $330 \mathrm{cfs}$. Between each trial, the cylindrical reservoir was refilled.

Three ADVs were used to obtain the velocity field measurements. Figure 6-2 displays the locations of ADV placement with respect to the jet array. The model distance between adjacent instruments is 15 in., with the exception of the instruments at the middle and front of the jet array where the distance between the ADVs in the longitudinal direction is $2 \mathrm{ft}$. Measurements were also taken with the ADVs at two different prototype depths: $4 \mathrm{ft}$, which corresponds with the shallowest depth at which the ADVs could function, and $9 \mathrm{ft}$, which represents the depth of a fully loaded barge. A series of linear actuators were used to vertically move the ADVs to the appropriate depths for each test. 
Figure 6-2. Location of ADVs, represented by $\mathbf{X}$, with respect to the jet array. The distance between adjacent instruments is 15 in.

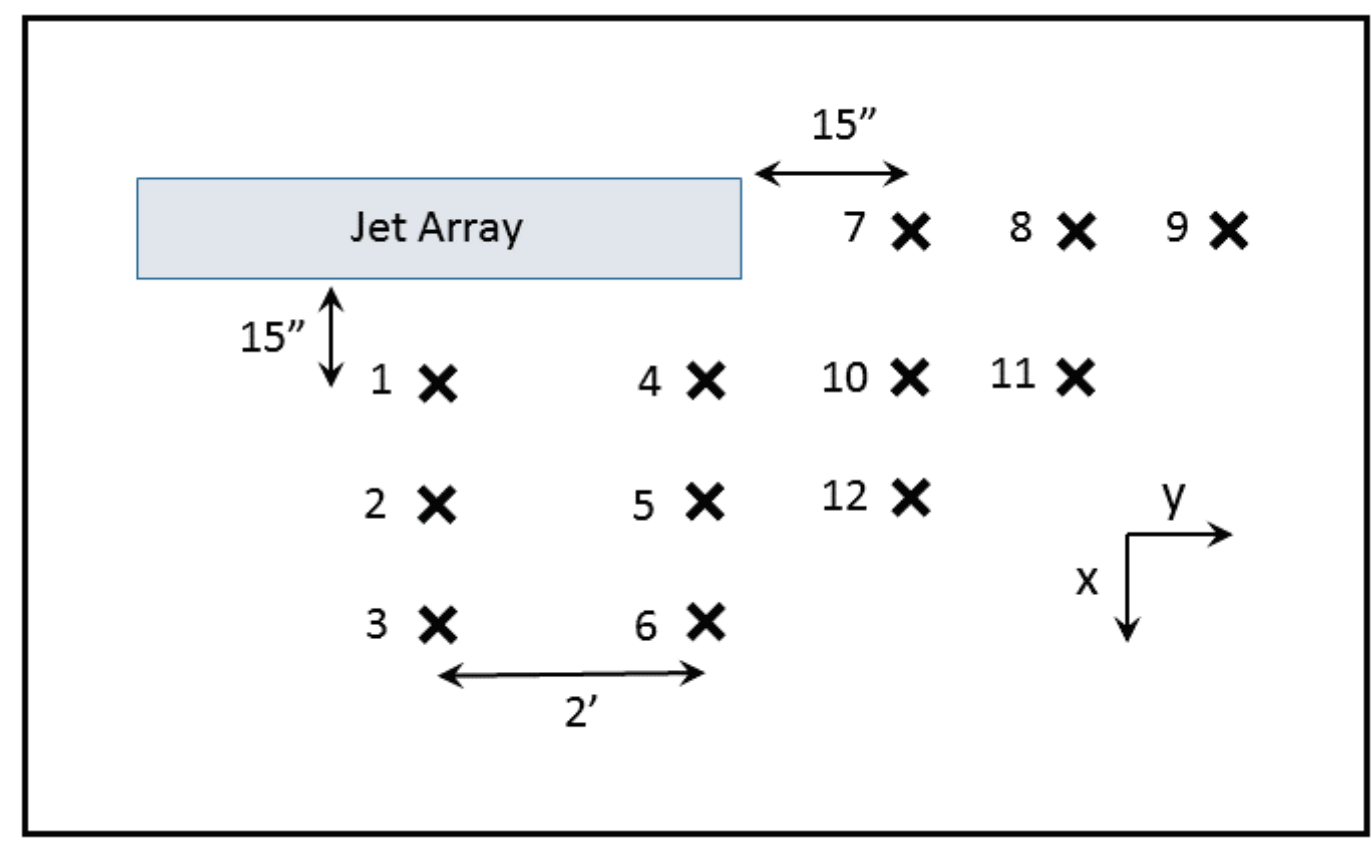

For each test, steady flow was reached before any measurements were taken. The measurements were taken until the cylindrical reservoir was empty, allowing for data collection time periods to range from approximately 5 to $10 \mathrm{~min}$, depending on the flow rate employed.

Average velocities were calculated for the horizontal $(\bar{u})$, longitudinal $(\bar{v})$, and vertical $(\bar{w})$ velocity components of the flow. To calculate the average velocities, averages of the velocity measurements were taken for continuous time periods modified to reflect the steady-state flow of the jets. Depending on the flow rate of the test, these time periods ranged in length from approximately 4 to $9 \mathrm{~min}$. The average velocities were then compared among the different ADV locations in the flow field.

\subsubsection{Flushing jet tests}

Before the model tow was navigated down the flume, the depth and the channel flow velocity were set to the desired values. The water temperature was measured because the model fish buoyancy is very sensitive to temperature variation.

At the start of the flushing jet test, the barge began its approach from the far end of the flume to reach the desired speed. As the barges approached 
but before reaching the jet array, the model fish were placed into the rake area by the boat operator seated in the barges. Twenty fish were used in each test run and counted to ensure a known number of fish were used. Once the fish were in place, cameras began recording the rake area to document the model fish movement throughout the test, and the jet pump was turned on. As the barge traveled over the jets, fish movement from the rake area was observed, and anything of note was recorded on the data sheet. Figure 6-3 shows a sample data testing sheet used to record the results for jet flushing testing. The time between when the tow's bow reached the edge of the barrier section and when the bow reached the end of the barrier section was measured to determine the tow speed. Video recordings of the tests were reviewed to accurately count the number of fish flushed and to check that the appropriate speed was achieved for each test.

Flushing jet testing was completed according to assessment levels ranging from the most favorable conditions for flushing to increasingly complex testing conditions. Successful results for a specific configuration at Level 1 allowed for it to be tested at Level 2, and so forth. This procedure was used to ensure that the test matrix did not grow unreasonably large with unsuccessful configurations being tested under conditions that make flushing less likely to occur.

Figure 6-3. Sample data sheet for jet flushing tests.

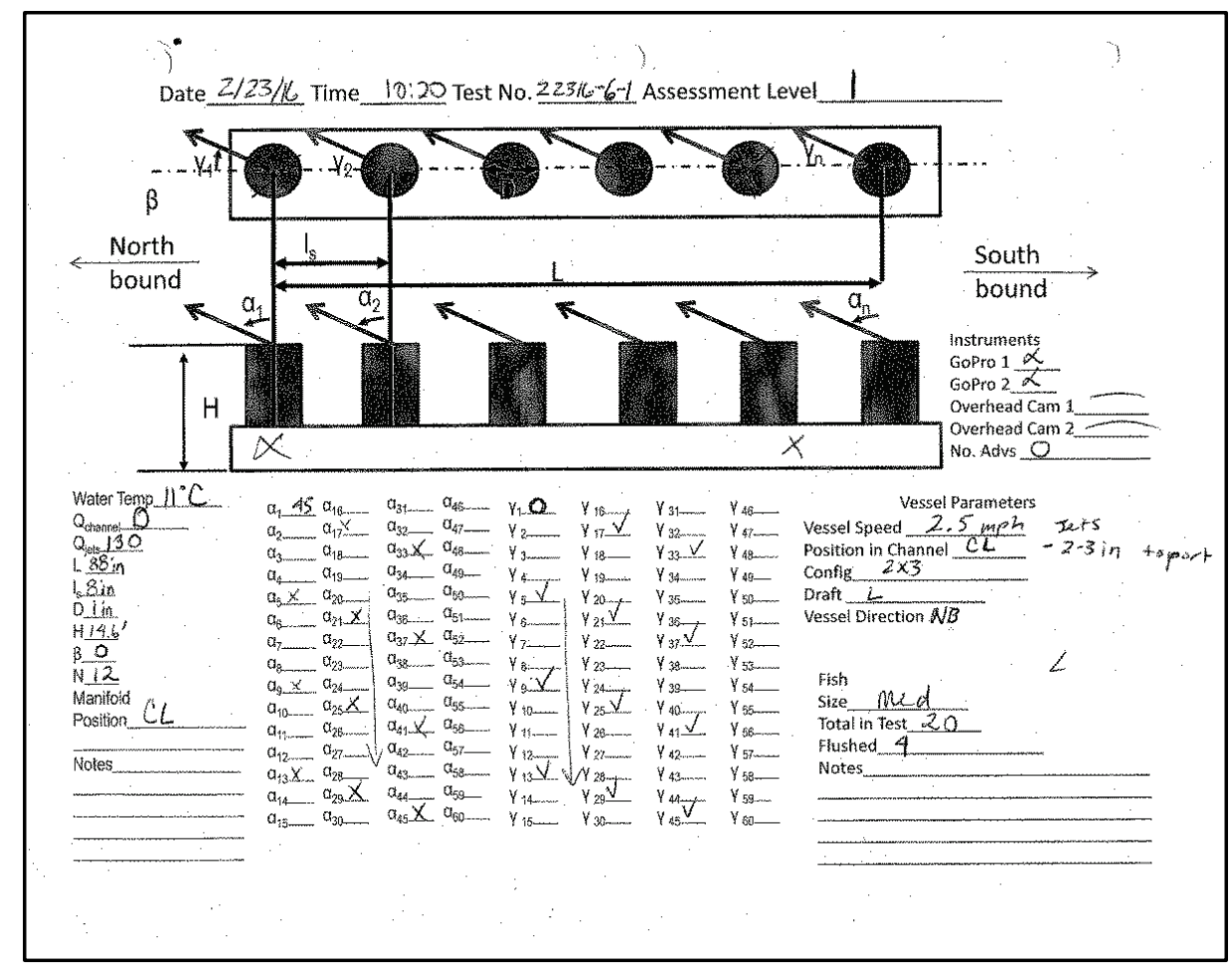




\subsection{Test matrix}

The test matrices in Table 6-1 and Table 6-2 outline the conditions for each test of flushing model fish from the rake area with maneuvers and jets, respectively. Some tests are described by the terminology chevron. A chevron configuration refers to the jets that started with a horizontal angle of o deg and slowly changed to an angle of $\pm 45 \mathrm{deg}$ by the end of the array with each jet turned opposite of the preceding one. Medium-sized model fish were used for most tests, and barge speeds of 2.5, 4, and $5 \mathrm{mph}$ were tested. A wide range of jet configurations was tested at $2.5 \mathrm{mph}$, and those that were found to be the most effective were chosen to test at the higher barge speeds. Small and large model fish were also used in later testing to understand the relationship of jet flushing effectiveness with fish size. Nearly all testing was completed using a 2-wide by 3-long model barge with a raked front and a forward-facing rake to flat junction between the second and third barges as the tested void area. Tests 20 to 23 were completed with different configurations to determine if configuration influenced jet flushing effectiveness. Tests 20 and 21 used barges that were turned around to determine if a backward-facing rake void area changed flushing effectiveness. Tests 22 and 23 used a rake-to-rake junction as the tested void area. Figure 6-4 shows the configurations used for the flushing tests.

Table 6-1. Testing matrix for maneuver flushing tests.

\begin{tabular}{|c|l|c|c|c|}
\hline $\begin{array}{c}\text { Test } \\
\text { Number }\end{array}$ & \multicolumn{1}{|c|}{ Description } & $\begin{array}{c}\text { Vessel Speed, } \\
\mathrm{mph}\end{array}$ & $\begin{array}{c}\text { Channel Flow } \\
\text { rate, cfs }\end{array}$ & Fish Size \\
\hline 1 & Swing-out w/tie off & $\mathrm{n} / \mathrm{a}$ & 0 & medium \\
\hline 2 & Swing-out w/o tie off & $\mathrm{n} / \mathrm{a}$ & 0 & medium \\
\hline 3 & Hard reverse & 2.5 & 0 & medium \\
\hline 4 & Hard reverse & 5 & 0 & medium \\
\hline 5 & Hard reverse from stop & $\mathrm{n} / \mathrm{a}$ & 0 & medium \\
\hline
\end{tabular}


Table 6-2. Testing matrix for jet flushing tests.

\begin{tabular}{|c|c|c|c|c|c|c|c|c|c|}
\hline 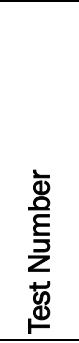 & 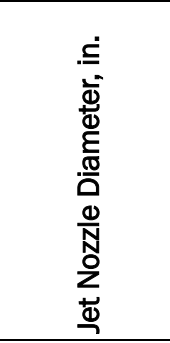 & 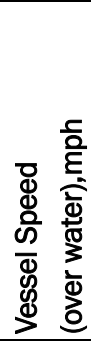 & 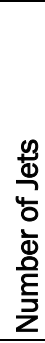 & 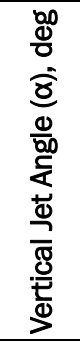 & 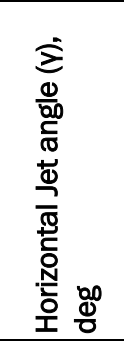 & 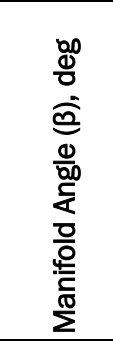 & 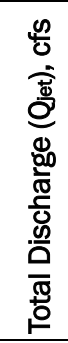 & 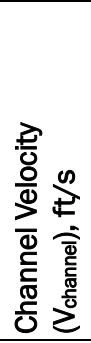 & 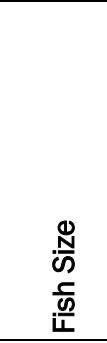 \\
\hline 6 & 16 & 2.5 & 12 & 45.0 & 0 & 0 & 330 & 0 & medium \\
\hline 7 & 16 & 2.5 & 12 & 67.5 & 0 & 0 & 330 & 0 & medium \\
\hline 8 & 12 & 2.5 & 12 & 45.0 & 0 & 0 & 330 & 0 & medium \\
\hline 9 & 12 & 2.5 & 12 & 67.5 & 0 & 0 & 330 & 0 & medium \\
\hline 10 & 8 & 2.5 & 12 & 45.0 & 0 & 0 & 330 & 0 & medium \\
\hline 11 & 8 & 2.5 & 12 & 67.5 & 0 & 0 & 330 & 0 & medium \\
\hline 12 & 12 & 2.5 & 12 & 67.5 & \pm 45 to 0 & 0 & 330 & 0 & medium \\
\hline 13 & 12 and 8 & 2.5 & 12 & 67.5 & \pm 45 to 0 & 0 & 330 & 0 & medium \\
\hline 14 & 8 & 2.5 & 12 & 67.5 & \pm 45 to 0 & 0 & 330 & 0 & medium \\
\hline 15 & 8 (chevron) & 2.5 & 12 & 67.5 & 0 & $+30 /-30$ & 330 & 0 & medium \\
\hline 16 & 12 (chevron) & 2.5 & 12 & 67.5 & 0 & $+30 /-30$ & 330 & 0 & medium \\
\hline 17 & 12 & 2.5 & 12 & 67.5 & 0 & 30 & 330 & 0 & medium \\
\hline 9_2 & 12 & 4 & 12 & 67.5 & 0 & 0 & 330 & 0 & medium \\
\hline $11 \_2$ & 8 & 4 & 12 & 67.5 & 0 & 0 & 330 & 0 & medium \\
\hline 12_2 & 12 & 4 & 12 & 67.5 & \pm 45 to 0 & 0 & 330 & 0 & medium \\
\hline 13_2 & 12 and 8 & 4 & 12 & 67.5 & \pm 45 to 0 & 0 & 330 & 0 & medium \\
\hline 9_3 & 12 & 4 & 18 & 67.5 & 0 & 0 & 330 & 0 & medium \\
\hline 11_3 & 8 & 4 & 18 & 67.5 & 0 & 0 & 330 & 0 & medium \\
\hline 12_3 & 12 & 4 & 18 & 67.5 & \pm 45 to 0 & 0 & 330 & 0 & medium \\
\hline 13_3 & 12 and 8 & 4 & 18 & 67.5 & \pm 45 to 0 & 0 & 330 & 0 & medium \\
\hline 9_4 & 12 & 4 & 18 & 67.5 & 0 & 0 & 330 & 0 & medium \\
\hline 11_4 & 8 & 4 & 18 & 67.5 & 0 & 0 & 330 & 0 & medium \\
\hline 12_4 & 12 & 4 & 18 & 67.5 & \pm 45 to 0 & 0 & 330 & 0 & medium \\
\hline 13_4 & 12 and 8 & 4 & 18 & 67.5 & \pm 45 to 0 & 0 & 330 & 0 & medium \\
\hline 9_5 & 12 & 4 & 18 & 67.5 & 0 & 0 & 430 & 0 & medium \\
\hline 11_5 & 8 & 4 & 18 & 67.5 & 0 & 0 & 430 & 0 & medium \\
\hline $12 \_5$ & 12 & 4 & 18 & 67.5 & \pm 45 to 0 & 0 & 430 & 0 & medium \\
\hline $13 \_5$ & 12 and 8 & 4 & 18 & 67.5 & \pm 45 to 0 & 0 & 430 & 0 & medium \\
\hline $9 \_6$ & 12 & 4 & 18 & 67.5 & 0 & 0 & 430 & 1.35 & medium \\
\hline 11_6 & 8 & 4 & 18 & 67.5 & 0 & 0 & 430 & 1.35 & medium \\
\hline
\end{tabular}




\begin{tabular}{|c|c|c|c|c|c|c|c|c|c|}
\hline 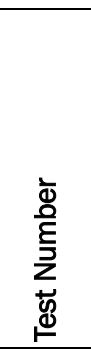 & 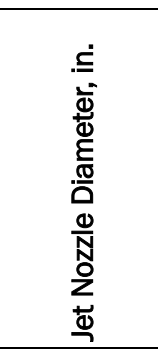 & 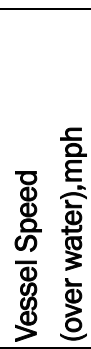 & 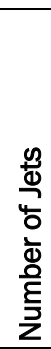 & 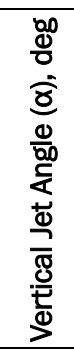 & 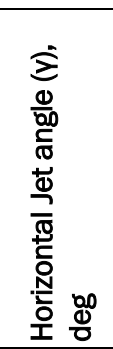 & 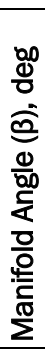 & 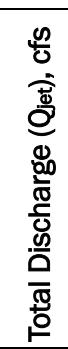 & 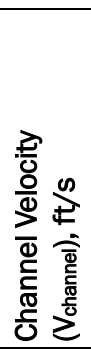 & 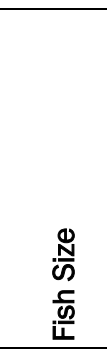 \\
\hline $12 \_6$ & 12 & 4 & 18 & 67.5 & \pm 45 to 0 & 0 & 430 & 1.35 & medium \\
\hline 13_6 & 12 and 8 & 4 & 18 & 67.5 & \pm 45 to 0 & 0 & 430 & 1.35 & medium \\
\hline 9_7 & 12 & 2.5 & 18 & 67.5 & 0 & 0 & 430 & 1.35 & medium \\
\hline 11_7 & 8 & 2.5 & 18 & 67.5 & 0 & 0 & 430 & 1.35 & medium \\
\hline $12 \_7$ & 12 & 2.5 & 18 & 67.5 & \pm 45 to 0 & 0 & 430 & 1.35 & medium \\
\hline 13_7 & 12 and 8 & 2.5 & 18 & 67.5 & \pm 45 to 0 & 0 & 430 & 1.35 & medium \\
\hline 9_8 & 12 & 4 & 18 & 67.5 & 0 & 0 & 430 & 0.56 & medium \\
\hline 11_8 & 8 & 4 & 18 & 67.5 & 0 & 0 & 430 & 0.56 & medium \\
\hline 9_9 & 12 & 2.5 & 18 & 67.5 & 0 & 0 & 430 & 0.56 & medium \\
\hline 11_9 & 8 & 2.5 & 18 & 67.5 & 0 & 0 & 430 & 0.56 & medium \\
\hline 18 & 8 & 2.5 & 18 & 67.5 & 0 & 0 & 430 & 0 & large \\
\hline 19 & 8 & 2.5 & 18 & 67.5 & 0 & 0 & 430 & 0 & small \\
\hline 18_2 & 8 & 4 & 18 & 67.5 & 0 & 0 & 430 & 0 & large \\
\hline 19_2 & 8 & 4 & 18 & 67.5 & 0 & 0 & 430 & 0 & small \\
\hline 9_10 & 12 & 5 & 18 & 67.5 & 0 & 0 & 430 & 0 & medium \\
\hline 11_10 & 8 & 5 & 18 & 67.5 & 0 & 0 & 430 & 0 & medium \\
\hline 12_10 & 12 & 5 & 18 & 67.5 & \pm 45 to 0 & 0 & 430 & 0 & medium \\
\hline 13_10 & 12 and $8 \mathrm{in}$. & 5 & 18 & 67.5 & \pm 45 to 0 & 0 & 430 & 0 & medium \\
\hline 9_11 & 12 & 5 & 18 & 67.5 & 0 & 0 & 430 & 0.56 & medium \\
\hline 11_11 & 8 & 5 & 18 & 67.5 & 0 & 0 & 430 & 0.56 & medium \\
\hline 9_12 & 12 & 5 & 18 & 67.5 & 0 & 0 & 430 & 1.35 & medium \\
\hline 11_12 & 8 & 5 & 18 & 67.5 & 0 & 0 & 430 & 0.56 & medium \\
\hline 18_3 & 8 & 5 & 18 & 67.5 & 0 & 0 & 430 & 0 & large \\
\hline 19_3 & 8 & 5 & 18 & 67.5 & 0 & 0 & 430 & 0 & small \\
\hline $20 *$ & 8 & 2.5 & 18 & 67.5 & 0 & 0 & 430 & 0 & medium \\
\hline $21^{*}$ & 8 & 5 & 18 & 67.5 & 0 & 0 & 430 & 0 & medium \\
\hline $22 * *$ & 8 & 2.5 & 18 & 67.5 & 0 & 0 & 430 & 0 & medium \\
\hline $23 * *$ & 8 & 5 & 18 & 67.5 & 0 & 0 & 430 & 0 & medium \\
\hline
\end{tabular}

*Backwards-facing rake void area used for tested void area

**Rake-to-rake junction used for tested void area 
Figure 6-4. Model barge configurations used for flushing tests.

Tests 1- 19 configuration

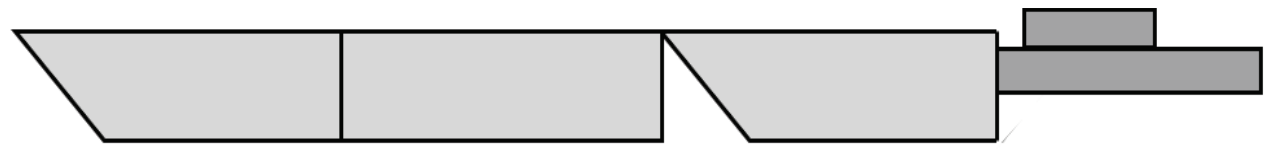

Tests 20-21

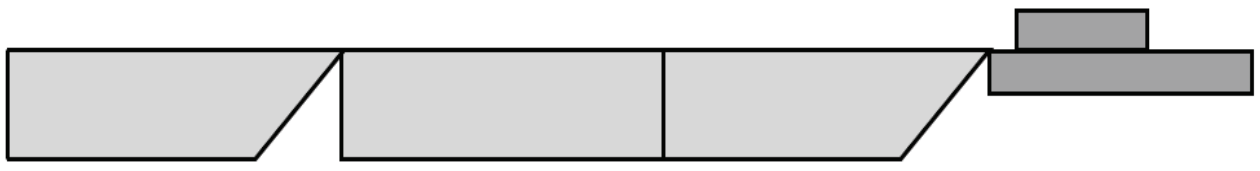

Tests 22-23

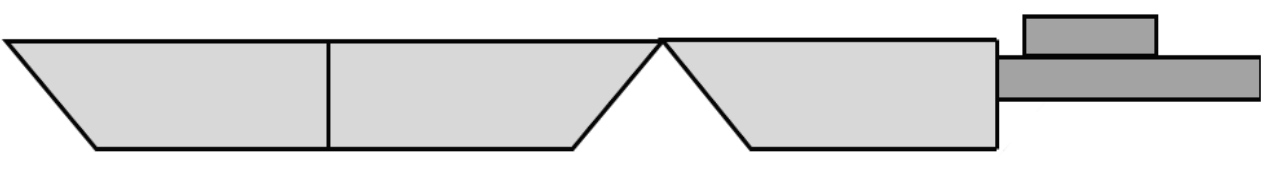

\subsection{Results}

For each test the percentage of fish flushed from the rake area was recorded. To more accurately express the effectiveness of a maneuver or jet configuration, the minimum effectiveness or lower bound of the $95 \%$ confidence interval (CI) on each test was calculated. This level is the minimum effectiveness that the testing indicates at $95 \%$ confidence. That is, based on the testing, the authors are $95 \%$ confident that the effectiveness is at least this effective. A minimum of three test tests with 20 fish each were necessary to calculate this value.

\subsubsection{Maneuvers}

Three maneuvers were tested for their effectiveness in clearing fish from the rake area. Table 6-3 shows the minimum effectiveness for each maneuver method. All the tested hard-reverse maneuvers had 100\% effectiveness, but the swing-out maneuvers were not as effective. 
Table 6-3. Results for maneuver flushing tests.

\begin{tabular}{|c|c|c|c|c|c|c|c|c|}
\hline \multirow[b]{2}{*}{ 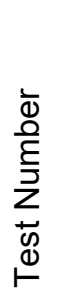 } & \multirow[b]{2}{*}{ 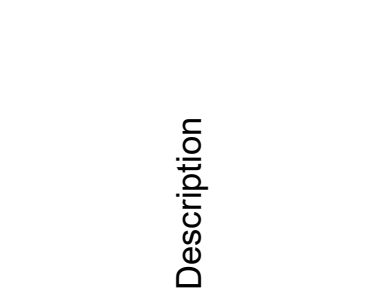 } & \multirow[b]{2}{*}{ 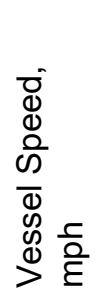 } & \multicolumn{5}{|c|}{ Flushing Proportion } & \multirow[b]{2}{*}{ 总 } \\
\hline & & & R1 & $\mathrm{R} 2$ & R3 & R4 & R5 & \\
\hline 1 & Swing-out w/tie off & $\mathrm{n} / \mathrm{a}$ & 0.4 & 0.15 & 0.7 & 0.1 & 0.7 & 0 \\
\hline 2 & Swing-out w/o tie off & $\mathrm{n} / \mathrm{a}$ & 0.35 & 0.7 & 0.15 & 0.15 & -- & 0 \\
\hline 3 & Hard Reverse & 2.5 & 1 & 1 & 1 & 1 & 1 & 1 \\
\hline 4 & Hard Reverse & 5 & 1 & 1 & 1 & 1 & 1 & 1 \\
\hline 5 & Hard Reverse from stop & $\mathrm{n} / \mathrm{a}$ & 1 & 1 & 1 & 1 & 1 & 1 \\
\hline
\end{tabular}

\subsubsection{Vertical jets}

Figure 6-5 displays the average velocities $(\bar{u})$ in the horizontal direction for the ADVs located at both $4 \mathrm{ft}$ and $9 \mathrm{ft}$ depths for Positions 1, 2, and 3 (as shown in Figure 6-2). Velocities obtained for this test are associated with the first jet array configuration. Figure 6-6 shows the average velocities at the same locations but for trials using the second jet array configuration. As shown, the overall average velocities tend to decrease as the horizontal distance from the jets increases for the first configuration, but the average velocities are more stable with distance from the jets for the second configuration. With regards to depth dependence, both configurations show that the velocities are higher near the surface ( $4 \mathrm{ft}$ depth) than lower in the water column ( $9 \mathrm{ft}$ depth). Overall, the average percent decrease in $\bar{u}$ from $4 \mathrm{ft}$ depth to $9 \mathrm{ft}$ depth was $39.5 \%$ for the first configuration and $56.5 \%$ for the second configuration. Alternatively, increasing the jet flow rate caused an increase in the average velocities. For the first jet configuration, almost doubling the flow rate from $130 \mathrm{cfs}$ to $250 \mathrm{cfs}$ resulted in an average percent increase of $330 \%$ in $\bar{u}$ at the surface. For the second jet configuration, the average percent increase in $\bar{u}$ at the surface was approximately $88 \%$. 
Figure 6-5. Average horizontal velocities $(\bar{u})$ for the first jet configuration with varying depths and flow rates at ADV Locations 1, 2, and 3.

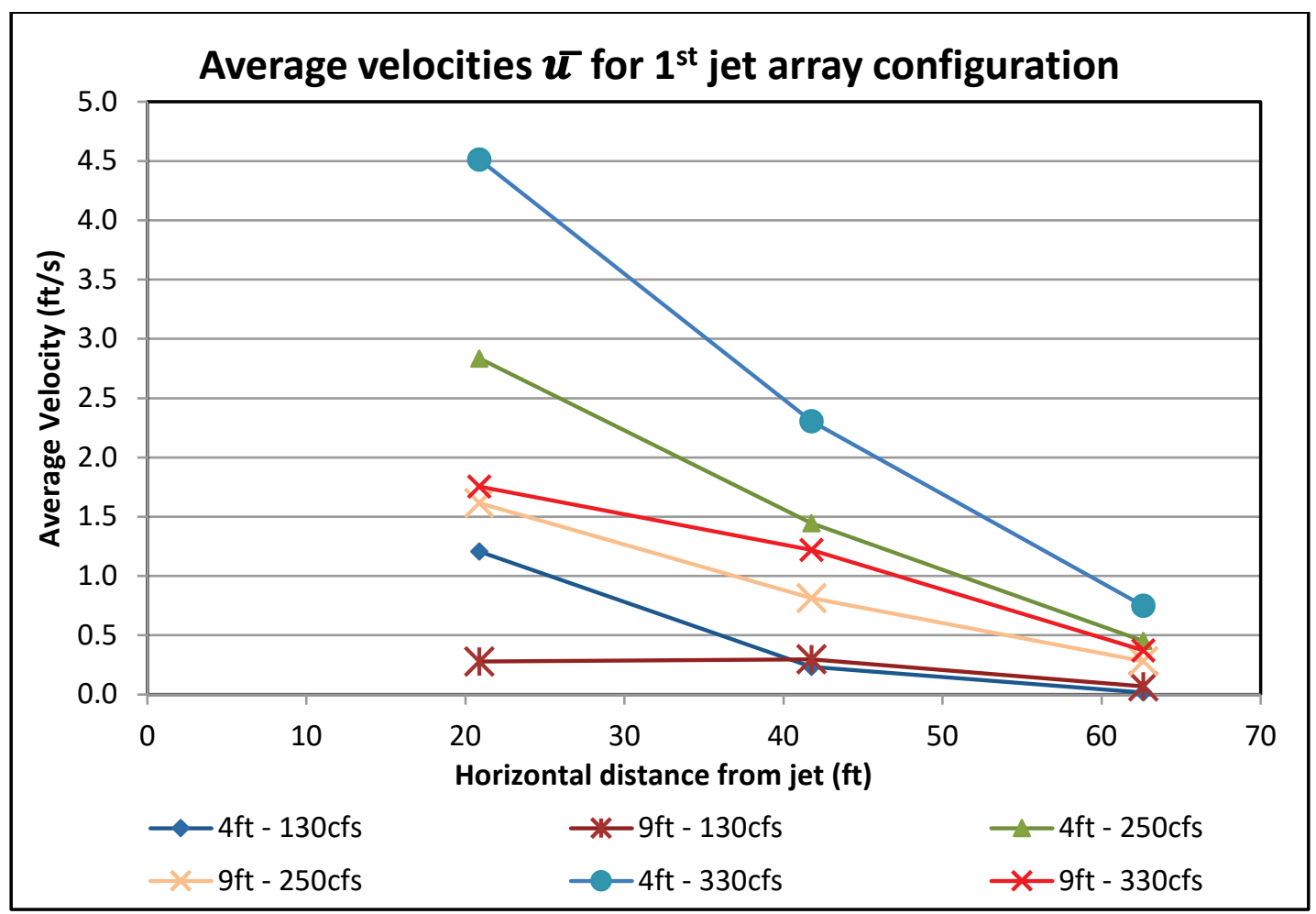

Figure 6-6. Average horizontal velocities for the second jet configuration with varying depths and flow rates at ADV Locations 1, 2, and 3.

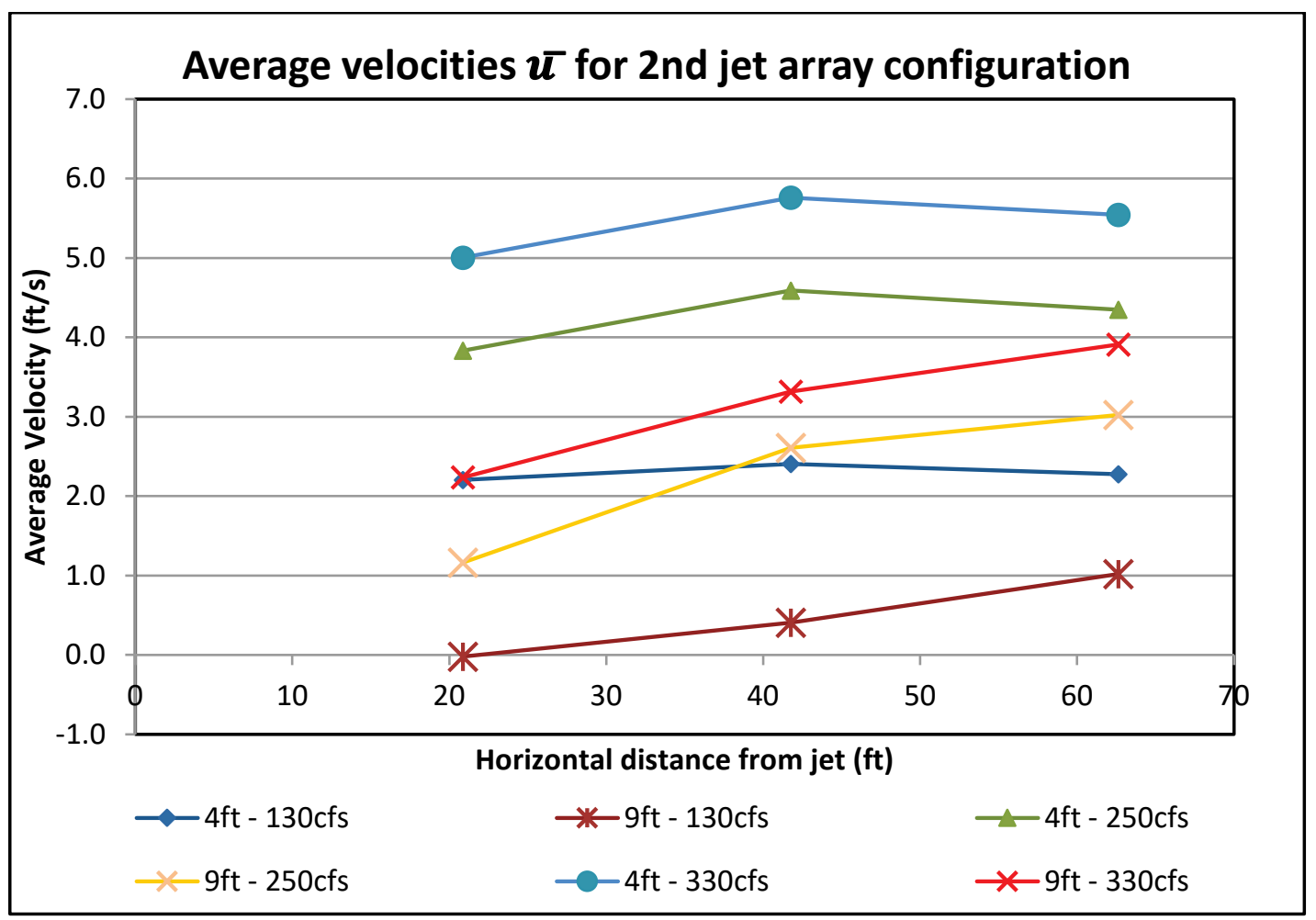


These trends are also present in the measurements obtained by the ADVs located at the end of the jet array, as indicated by Positions 4, 5, and 6 in Figure 6-2. Figure 6-7 presents the average velocities in the horizontal direction for differing depths and flow rates. For the tests in question, the second jet array was employed. As shown in the Figure 6-7, average velocities are higher near the surface ( $4 \mathrm{ft}$ depth), with the average percent decrease in $\bar{u}$ from $4 \mathrm{ft}$ depth to $9 \mathrm{ft}$ depth being approximately $130 \%$. This large number reflects the change in polarity from positive velocities at $4 \mathrm{ft}$ depth to negative velocities at $9 \mathrm{ft}$ depth. With regards to flow rate, increasing the flow rate from $130 \mathrm{cfs}$ to $250 \mathrm{cfs}$ produced an average percent increase in $\bar{u}$ at the surface of $140 \%$.

Figure 6-7. Average horizontal velocities for the second jet configuration with varying depths and flow rates at ADV Locations 4, 5, and 6.

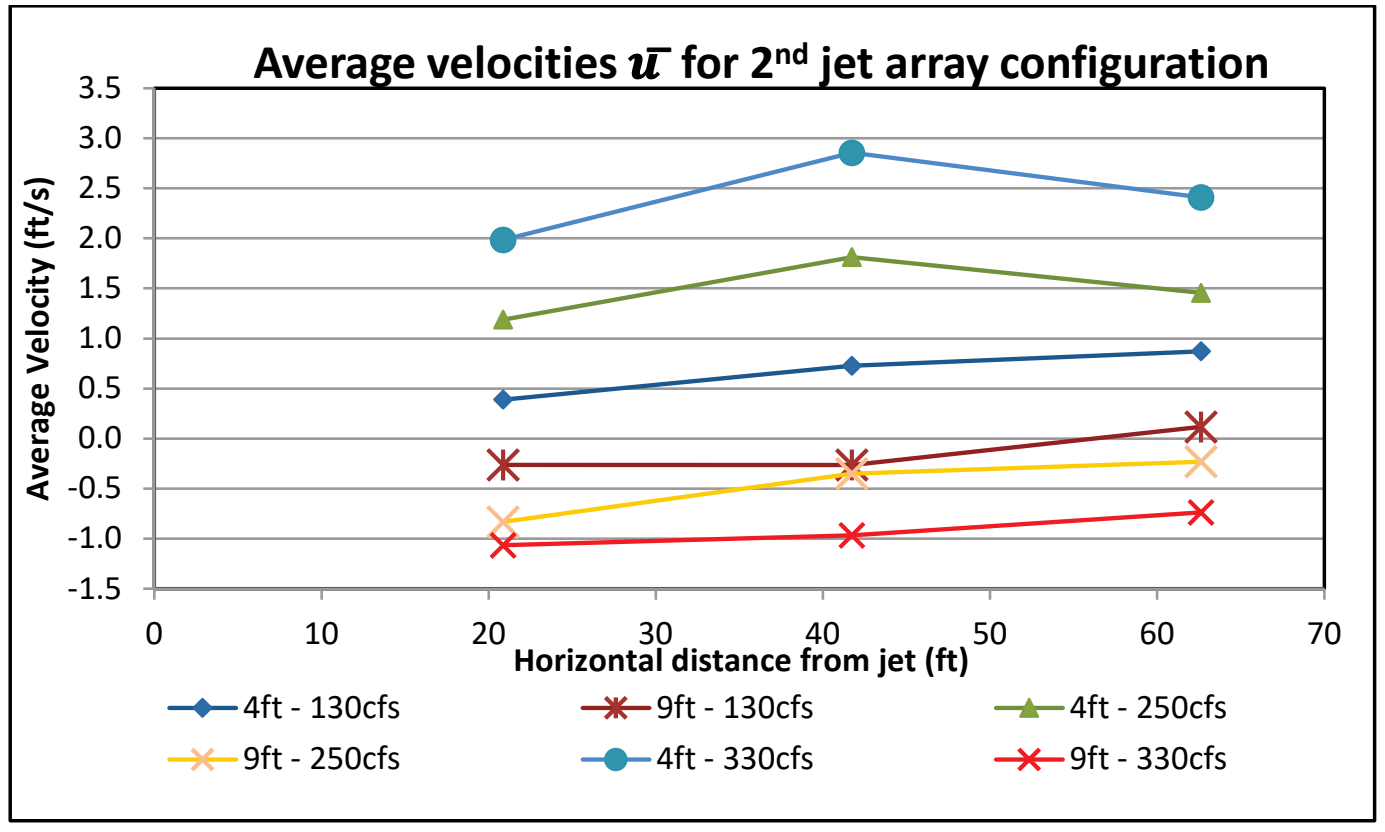

Figure 6-8 displays the average velocities in the longitudinal direction $(\bar{v})$ for the ADVs located at 4ft depth for positions 7,8 , and 9 as shown in Figure 6.2. Velocities obtained for this test run are associated with the second jet array configuration. Figure 6-9 shows the average velocities for the same test conditions, with the exception that the results are associated with ADVs placed at locations 1, 4, 10, and 11. Here can be seen that the average velocities decrease from positive to negative values as the location moves from the center of the jet toward the back of the jet. As the distance from jet center continues to increase, $\bar{v}$ continues to hold a negative value but begins to increase. This trend is also seen in Figure 6.8, where the average velocities are shown to increase as the distance from the jet increases. 
Figure 6-8. Average longitudinal velocities for the second jet configuration with instruments at a $4 \mathrm{ft}$ depth and at ADV Locations 7, 8, and 9.

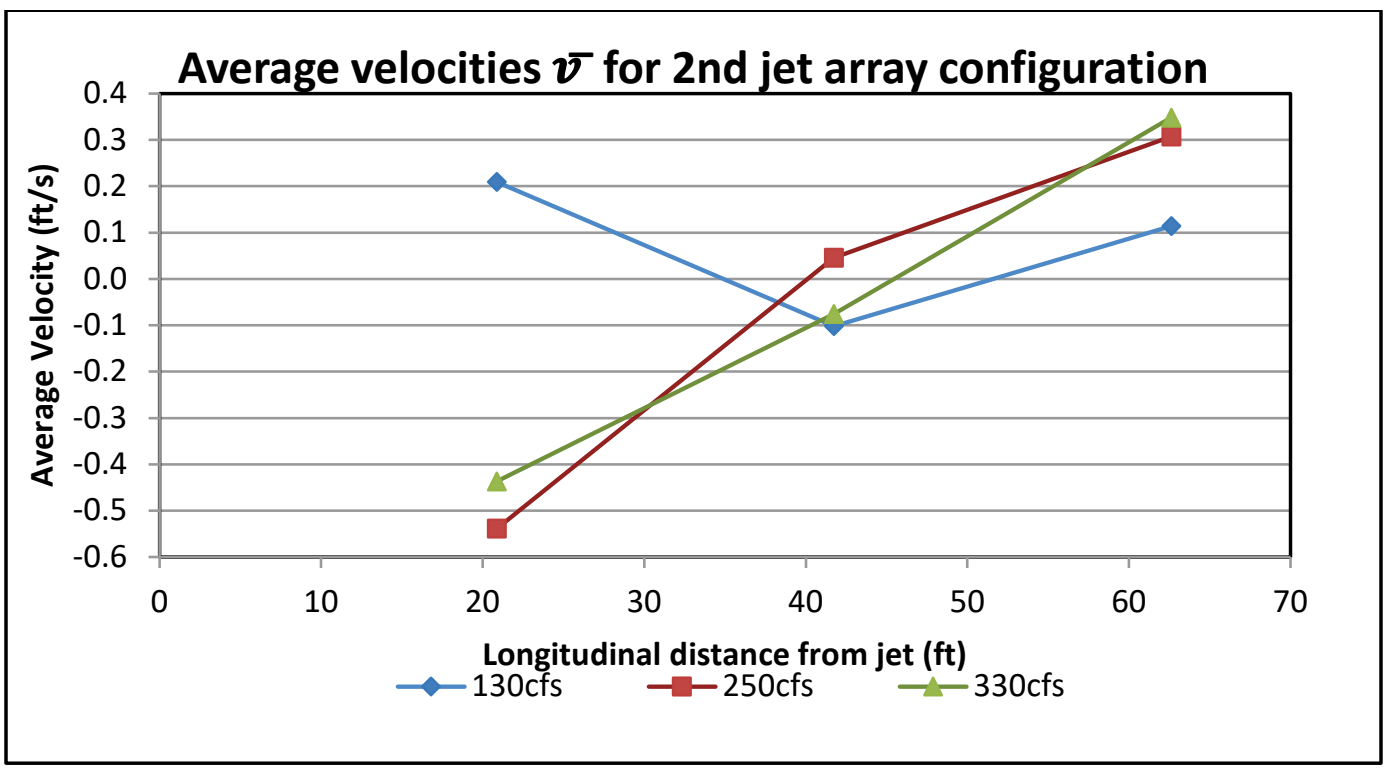

Figure 6-9. Average longitudinal velocities for the second jet configuration with instruments at a $4 \mathrm{ft}$ depth and at ADV Locations 1, 4, 10.

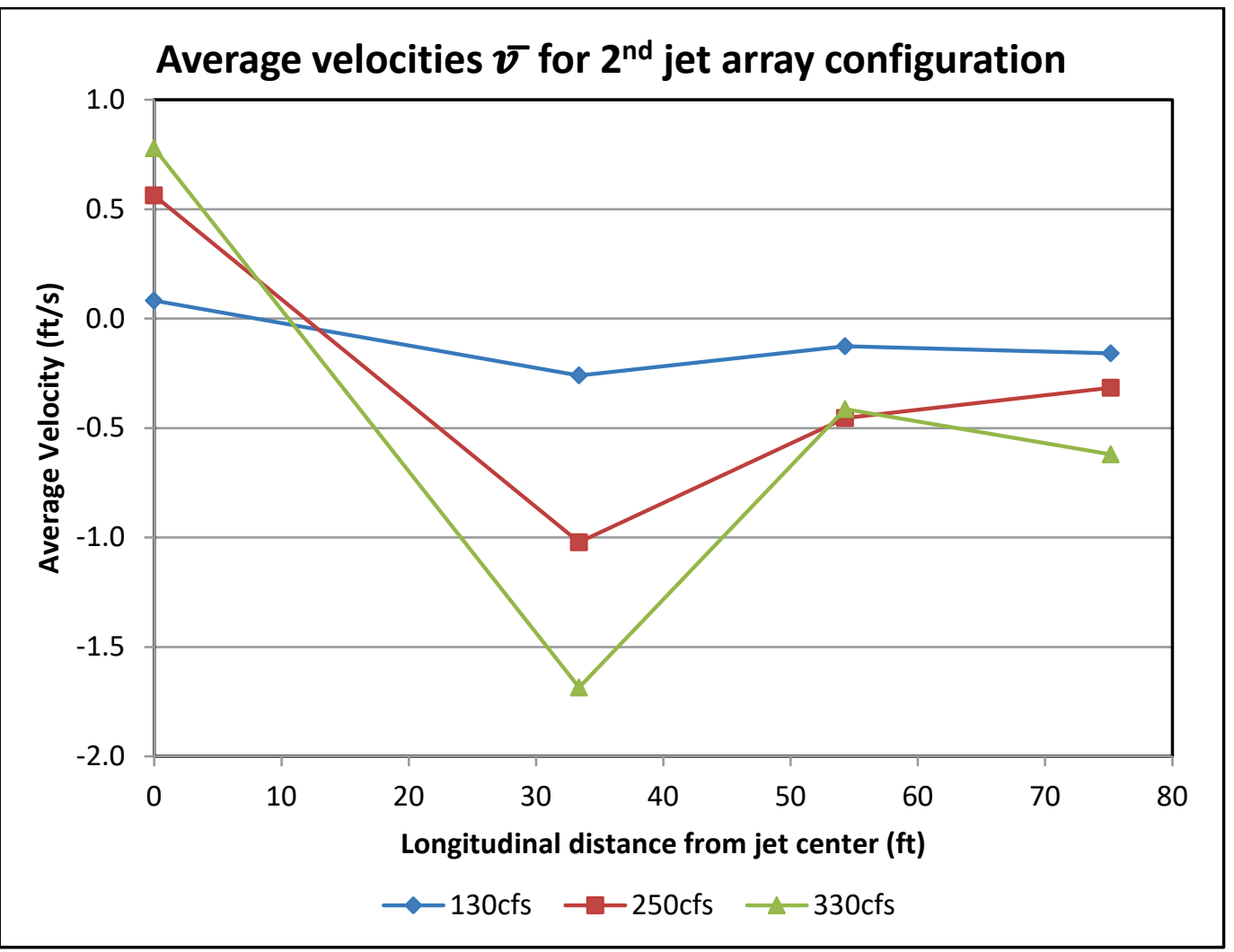




\subsubsection{Jet flushing}

Flushing jets were tested according to Assessment Levels 1 through 4. Assessment Level 1 was tested for the low barge speed setting, $2.5 \mathrm{mph}$. Those configurations that performed well at Level 1 were tested further at Level 2 in which the barge velocity was increased. At Level 3 the total jet flow rate was increased, and finally, the field of configurations was further narrowed for Level 4 testing in which two channel flow rates were tested, fish size was varied, and two additional barge configurations were tested.

\subsubsection{Level 1: Base level testing}

Initially, jet configurations consisting of 12 jets with vertical angles of $45 \mathrm{deg}$ or $67.5 \mathrm{deg}$ and nozzle prototype diameters of 8,12 , or $16 \mathrm{in}$. were tested to clear fish from the rake area of barge traveling at $2.5 \mathrm{mph}$. Table 6-4 shows the results of each run as well as the estimated minimum effectiveness at the $95 \% \mathrm{CI}$. Tests 1-5 are not presented as they represent the test that used maneuvers to flush the fish and detailed in Section 6.3.1. Level 1 testing did not include multiple replications for all tests, so some of the tests at this level did not meet the data criteria to calculate the minimum effectiveness at a $95 \% \mathrm{CI}$. More details on the configurations can be found in Table 6-2.

Table 6-4. Results for Level 1 jet flushing tests.

\begin{tabular}{|c|c|c|c|c|c|c|c|c|c|c|}
\hline \multirow[b]{2}{*}{ 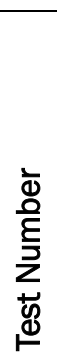 } & \multirow{2}{*}{ 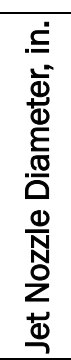 } & \multirow[b]{2}{*}{ 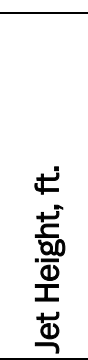 } & \multirow[b]{2}{*}{ 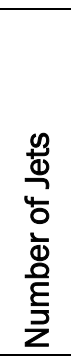 } & \multirow[b]{2}{*}{ 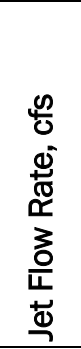 } & \multirow[b]{2}{*}{ 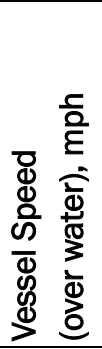 } & \multirow{2}{*}{ 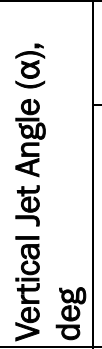 } & \multicolumn{3}{|c|}{$\begin{array}{l}\text { Flushing } \\
\text { Proportion }\end{array}$} & \multirow[b]{2}{*}{ 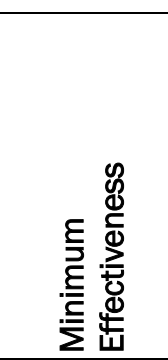 } \\
\hline & & & & & & & R1 & $\mathrm{R} 2$ & R3 & \\
\hline 6 & 16 & 14.6 & 12 & 330 & 2.5 & 45.0 & 0.3 & 0.6 & & $\begin{array}{c}\text { Insufficient } \\
\text { data }\end{array}$ \\
\hline 7 & 16 & 14.6 & 12 & 330 & 2.5 & 67.5 & 0.55 & & & $\begin{array}{c}\text { Insufficient } \\
\text { data }\end{array}$ \\
\hline 8 & 12 & 14.6 & 12 & 330 & 2.5 & 45.0 & 0.95 & 1 & & $\begin{array}{c}\text { Insufficient } \\
\text { data }\end{array}$ \\
\hline 9 & 12 & 14.6 & 12 & 330 & 2.5 & 67.5 & 1 & 1 & 1 & 1 \\
\hline 10 & 8 & 13.6 & 12 & 330 & 2.5 & 45.0 & 1 & 1 & 0.85 & 0.70 \\
\hline 11 & 8 & 13.6 & 12 & 330 & 2.5 & 67.5 & 1 & 1 & & $\begin{array}{l}\text { Insufficient } \\
\text { data }\end{array}$ \\
\hline 12 & 12 & 14.6 & 12 & 330 & 2.5 & 67.5 & 1 & 1 & 1 & 1 \\
\hline
\end{tabular}




\begin{tabular}{|c|c|c|c|c|c|c|c|c|c|c|}
\hline \multirow[b]{2}{*}{ 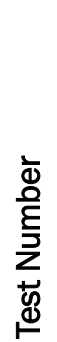 } & \multirow{2}{*}{ 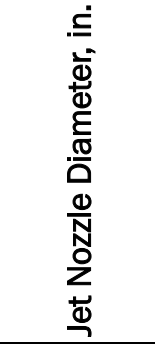 } & \multirow[b]{2}{*}{ 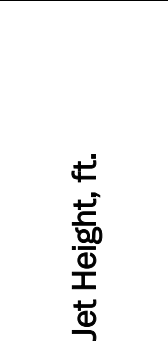 } & \multirow[b]{2}{*}{ 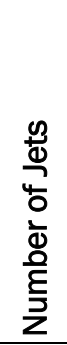 } & \multirow{2}{*}{ 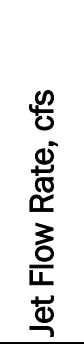 } & \multirow{2}{*}{ 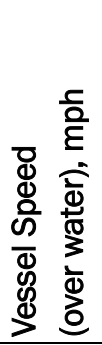 } & \multirow{2}{*}{ 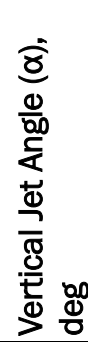 } & \multicolumn{3}{|c|}{$\begin{array}{l}\text { Flushing } \\
\text { Proportion }\end{array}$} & \multirow[b]{2}{*}{ 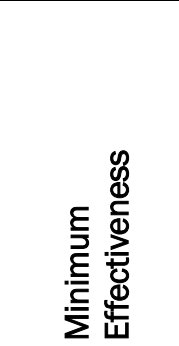 } \\
\hline & & & & & & & R1 & R2 & R3 & \\
\hline 13 & 12 and 8 & 13.6/14.6 & 12 & 330 & 2.5 & 67.5 & 1 & 1 & 1 & 1 \\
\hline 14 & 8 & 13.6 & 12 & 330 & 2.5 & 67.5 & 1 & $\begin{array}{c}0.9 \\
5\end{array}$ & 0.9 & 0.80 \\
\hline 15 & $\begin{array}{c}8 \\
\text { (chevron) }\end{array}$ & 13.6 & 12 & 330 & 2.5 & 67.5 & 0.05 & & & $\begin{array}{c}\text { Insufficient } \\
\text { Data }\end{array}$ \\
\hline 16 & $\begin{array}{c}12 \\
\text { (chevron) }\end{array}$ & 14.6 & 12 & 330 & 2.5 & 67.5 & 0.05 & & & $\begin{array}{l}\text { Insufficient } \\
\text { Data }\end{array}$ \\
\hline 17 & 12 & 14.6 & 12 & 330 & 2.5 & 67.5 & 0.75 & $\begin{array}{c}0.1 \\
5\end{array}$ & & $\begin{array}{c}\text { Insufficient } \\
\text { Data }\end{array}$ \\
\hline
\end{tabular}

\subsubsection{Level 2: Increased barge speed and jet array length}

Configurations 9, 11, 12, and 13 were tested with a barge speed of $5 \mathrm{mph}$ with each configuration having a total of 12 jets and jet flow rate of $330 \mathrm{cfs}$. The number of jets was increased to 18 to increase the jet exposure time starting with Tests 9-3, 11-3, 12-3, and 13-3. Furthermore, the speed was decreased to $4 \mathrm{mph}$ for Tests 9-4, 11-4, 12-4, and 13-4 as a means of increasing exposure time. The results of Level 2 assessment are listed in Table 6-5. 
Table 6-5. Results of Level 2 jet flushing tests.

\begin{tabular}{|c|c|c|c|c|c|c|c|c|c|c|c|c|}
\hline \multirow[b]{2}{*}{ 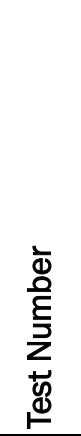 } & \multirow[b]{2}{*}{ 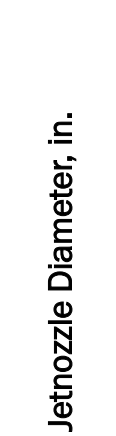 } & \multirow[b]{2}{*}{ 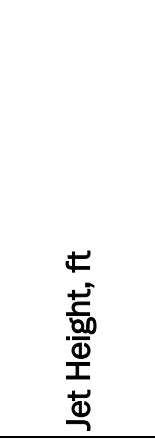 } & \multirow[b]{2}{*}{ 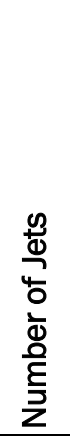 } & \multirow[b]{2}{*}{ 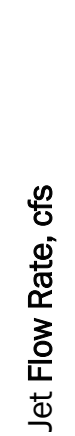 } & \multirow[b]{2}{*}{ 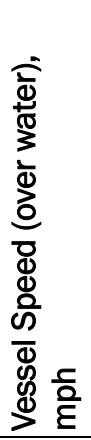 } & \multirow[b]{2}{*}{ 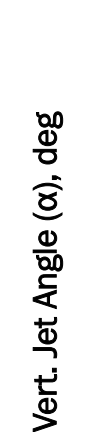 } & \multicolumn{5}{|c|}{ Flushing Proportion } & \multirow[b]{2}{*}{ 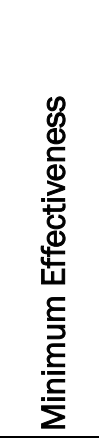 } \\
\hline & & & & & & & R1 & R2 & R3 & R4 & R5 & \\
\hline 9_2 & 12 & 14.6 & 12 & 330 & 5 & 67.5 & 0.5 & 0.45 & 0.25 & & & 0.014 \\
\hline 11_2 & 8 & 13.6 & 12 & 330 & 5 & 67.5 & 0.15 & 0.5 & 0.65 & 0.75 & & 0 \\
\hline 12_2 & 12 & 14.6 & 12 & 330 & 5 & 67.5 & 0.35 & 0.1 & 0.1 & & & 0 \\
\hline 13_2 & $\begin{array}{c}12 \text { and } \\
8 \text { in. }\end{array}$ & $13.6 / 14.6$ & 12 & 330 & 5 & 67.5 & 0.4 & 0.1 & 0.15 & & & 0 \\
\hline 9_3 & 12 & 14.6 & 18 & 330 & 5 & 67.5 & 0.45 & 0.35 & 0.35 & & & 0.22 \\
\hline 11_3 & 8 & 13.6 & 18 & 330 & 5 & 67.5 & 0.25 & 0.4 & 0.55 & 0.35 & 0.4 & 0.16 \\
\hline 12_3 & 12 & 14.6 & 18 & 330 & 5 & 67.5 & 0.2 & 0.15 & 0.35 & & & 0 \\
\hline 13_3 & $\begin{array}{c}12 \text { and } \\
8\end{array}$ & $13.6 / 14.6$ & 18 & 330 & 5 & 67.5 & 0.45 & 0.25 & 0.2 & & & 0 \\
\hline 9_4 & 12 & 14.6 & 18 & 330 & 4 & 67.5 & 0.3 & 0.2 & 0.2 & & & 0.065 \\
\hline 11_4 & 8 & 13.6 & 18 & 330 & 4 & 67.5 & 0.45 & 0.1 & 0.35 & & & 0 \\
\hline 12_4 & 12 & 14.6 & 18 & 330 & 4 & 67.5 & 0.15 & 0.3 & 0.15 & & & 0 \\
\hline 13_4 & $\begin{array}{c}12 \text { and } \\
8\end{array}$ & $13.6 / 14.6$ & 18 & 330 & 4 & 67.5 & 0.15 & 0.2 & 0.2 & & & 0.099 \\
\hline
\end{tabular}

\subsubsection{Level 3: Increased jet flow}

At Level 3 of testing, Configurations 9, 11, 12, and 13 were tested at both $4 \mathrm{mph}$ and $5 \mathrm{mph}$ with an increased prototype total jet flow rate of $430 \mathrm{cfs}$. The flow rate was increased to counter the decrease in the individual jet velocity when the jet array length was increased from 12 jet nozzles to 18 jet nozzles. The results of Level 3 assessment are listed in Table 6-6. Figure 6-10 shows the minimum flushing effectiveness at the $95 \%$ confidence interval for Level 3 testing. 
Table 6-6. Results of Level 3 jet flushing tests.

\begin{tabular}{|c|c|c|c|c|c|c|c|c|c|c|}
\hline \multirow[b]{2}{*}{ 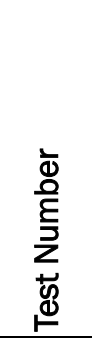 } & \multirow{2}{*}{ 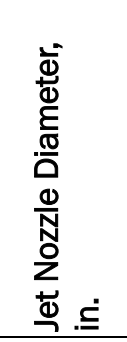 } & \multirow[b]{2}{*}{ 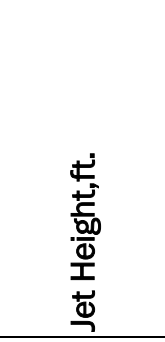 } & \multirow[b]{2}{*}{ 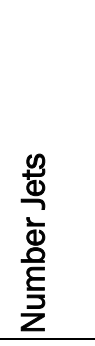 } & \multirow[b]{2}{*}{ 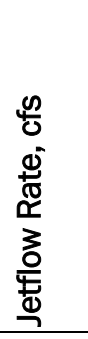 } & \multirow[b]{2}{*}{ 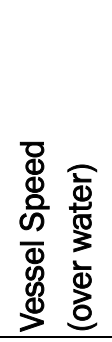 } & \multirow{2}{*}{ 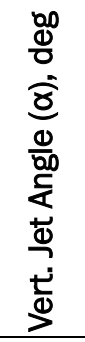 } & \multicolumn{3}{|c|}{ Flushing Proportion } & \multirow[b]{2}{*}{ 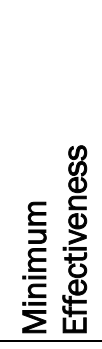 } \\
\hline & & & & & & & R1 & R2 & R3 & \\
\hline $9-5$ & 12 & 14.6 & 18 & 430 & 4 & 67.5 & 0.65 & 0.8 & 0.85 & 0.46 \\
\hline $11-5$ & 8 & 13.6 & 18 & 430 & 4 & 67.5 & 0.9 & 1 & 0.95 & 0.80 \\
\hline $12-5$ & 12 & 14.6 & 18 & 430 & 4 & 67.5 & 0.95 & 1 & 0.8 & 0.61 \\
\hline $13-5$ & 12 and 8 & 13.6/14.6 & 18 & 430 & 4 & 67.5 & 0.95 & 0.8 & 0.85 & 0.64 \\
\hline $9-10$ & 12 & 14.6 & 18 & 430 & 5 & 67.5 & 0.72 & 0.3 & 0.65 & 0 \\
\hline $11-10$ & 8 & 13.6 & 18 & 430 & 5 & 67.5 & 0.45 & 0.65 & 0.85 & 0.066 \\
\hline $12-10$ & 12 & 14.6 & 18 & 430 & 5 & 67.5 & 0.6 & 0.4 & 0.65 & 0.16 \\
\hline $13-10$ & 12 and 8 & 13.6/14.6 & 18 & 430 & 5 & 67.5 & 0.75 & 0.5 & 0.4 & 0.024 \\
\hline
\end{tabular}

Figure 6-10. Minimum flushing effectiveness for medium fish at $4 \mathrm{mph}$ and $5 \mathrm{mph}$.

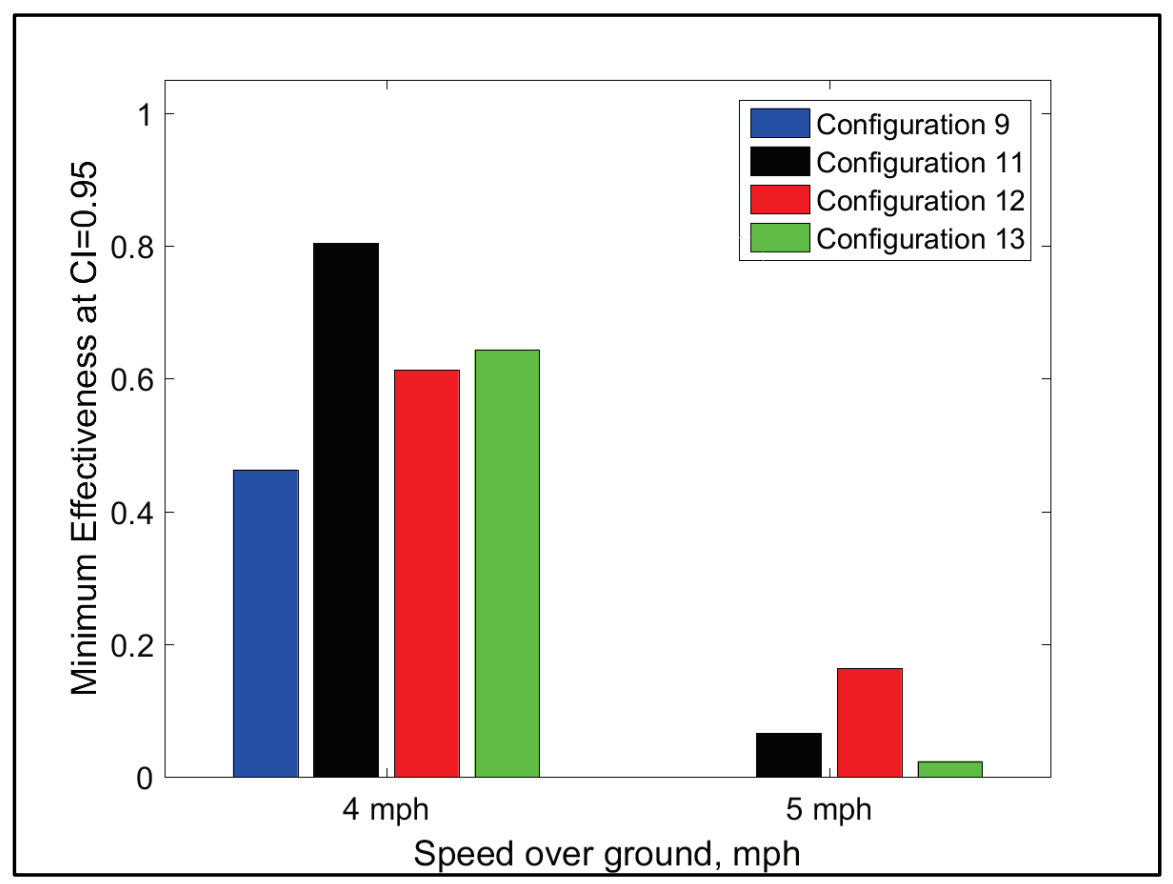

\subsubsection{Level 4: Channel flow introduced}

At the final assessment level, the impact of a channel flow rate on jet flushing was tested. Tests were run with prototype canal flow rates of $2,500 \mathrm{cfs}$ and $6,000 \mathrm{cfs}$, which represent the 5oth percentile and 95th percentile of flows measured in the prototype channel, respectively. 
A 5 mph (over-water) barge speed was tested at this assessment level. The over-water barge speed is unchanged, but the over-ground speed was reduced as the barge is traveling in the opposite direction of channel flow. Configurations 9 and 11 were used because (on average) they represent the least and most successful configurations, respectively. The results of Level 4 assessment are listed in Table 6-7. Figure 6-11 shows the minimum effective flushing vs. the channel flow rate.

Table 6-7. Results of Level 4 jet flushing tests.

\begin{tabular}{|c|c|c|c|c|c|c|c|c|c|c|}
\hline \multirow[b]{2}{*}{ 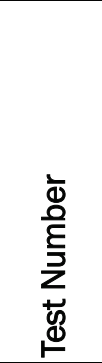 } & \multirow{2}{*}{ 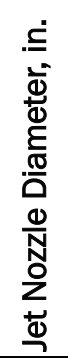 } & \multirow[b]{2}{*}{ 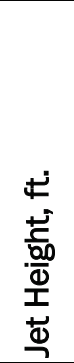 } & \multirow[b]{2}{*}{ 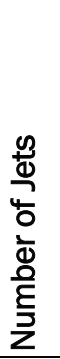 } & \multirow[b]{2}{*}{ 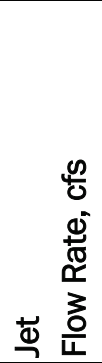 } & \multirow[b]{2}{*}{ 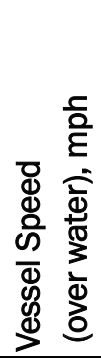 } & \multirow[b]{2}{*}{ 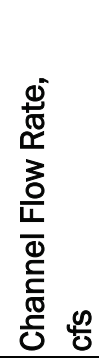 } & \multicolumn{3}{|c|}{ Flushing Proportion } & \multirow{2}{*}{ 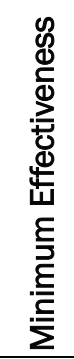 } \\
\hline & & & & & & & R1 & R2 & R3 & \\
\hline $9-11$ & 12 & 14.6 & 18 & 430 & 5 & 2,500 & 0.45 & 0.70 & 0.50 & 0.16 \\
\hline $11-11$ & 8 & 13.6 & 18 & 430 & 5 & 2,500 & 0.75 & 0.65 & 0.70 & 0.55 \\
\hline $9-12$ & 12 & 14.6 & 18 & 430 & 5 & 6,000 & 0.60 & 0.80 & 0.60 & 0.33 \\
\hline $11-12$ & 8 & 13.6 & 18 & 430 & 5 & 6,000 & 0.75 & 0.75 & 0.65 & 0.55 \\
\hline
\end{tabular}

Figure 6-11. Minimum flushing effectiveness vs. channel flow rate.

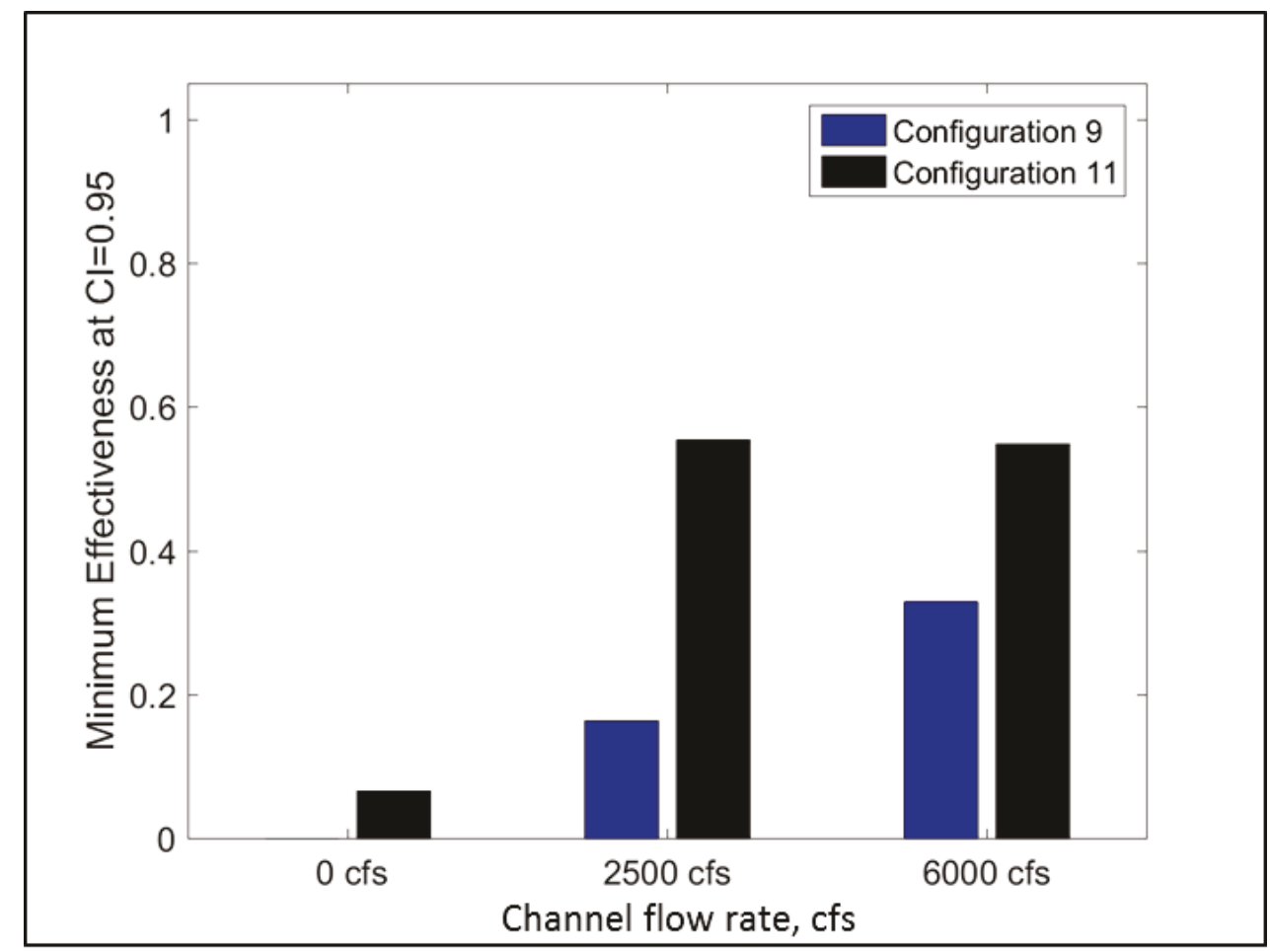


Previously mentioned testing was conducted using the medium-sized model fish. Tests were then conducted with small- and large-sized model fish to determine the effect of fish size on flushing effectiveness. On average, the configuration used in Test 11 was best at flushing model fish from the rake and was used to test the small- and large-sized fish at both $2.5 \mathrm{mph}$ and $5 \mathrm{mph}$ with no flow in the channel. The prototype nozzle diameter was $12 \mathrm{in}$., and the jet height was $14.6 \mathrm{ft}$. The results for small and large fish are presented in Table 6-8. Figure 6-12 shows the minimum effective flushing for different fish sizes. Additionally, two additional barge configurations (Figure 6-4) were tested to understand the effects of reversing the orientation of the rake-flat junction (Tests 20-21) and a rakerake junction (Tests 22-23) at both $2.5 \mathrm{mph}$ and $5 \mathrm{mph}$. These tests were completed using medium-sized model fish with Configuration 11. The results for the alternate barge configurations are listed in Table 6-9.

Table 6-8. Results of Level 4 jet flushing tests with large and small model fish.

\begin{tabular}{|c|c|c|c|c|c|c|c|c|c|}
\hline \multirow[b]{2}{*}{ 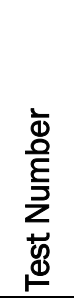 } & \multirow[b]{2}{*}{$\begin{array}{l}\stackrel{N}{N} \\
\frac{N}{\omega} \\
\frac{5}{\omega 0} \\
\frac{0}{4}\end{array}$} & \multirow[b]{2}{*}{ 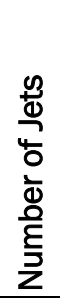 } & \multirow{2}{*}{ 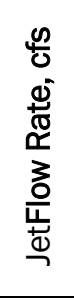 } & \multirow{2}{*}{ 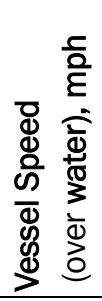 } & \multirow{2}{*}{ 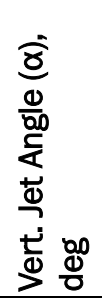 } & \multicolumn{3}{|c|}{ Flushing Proportion } & \multirow[b]{2}{*}{ 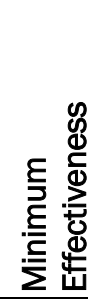 } \\
\hline & & & & & & R1 & $\mathrm{R} 2$ & R3 & \\
\hline 18 & Large & 18 & 430 & 2.5 & 67.5 & 1 & 1 & 1 & 1 \\
\hline 19 & Small & 18 & 430 & 2.5 & 67.5 & 1 & 1 & 1 & 1 \\
\hline $18-3$ & Large & 18 & 430 & 4 & 67.5 & 0.8 & 0.8 & 0.85 & 0.73 \\
\hline $19-3$ & Small & 18 & 430 & 4 & 67.5 & 0.95 & 1 & 0.95 & 0.88 \\
\hline $18-3$ & Large & 18 & 430 & 5 & 67.5 & 0.63 & 0.72 & 0.66 & 0.53 \\
\hline $19-3$ & Small & 18 & 430 & 5 & 67.5 & 0.9 & 0.9 & 0.3 & 0 \\
\hline
\end{tabular}


Figure 6-12. Minimum flushing effectiveness vs. model fish size.

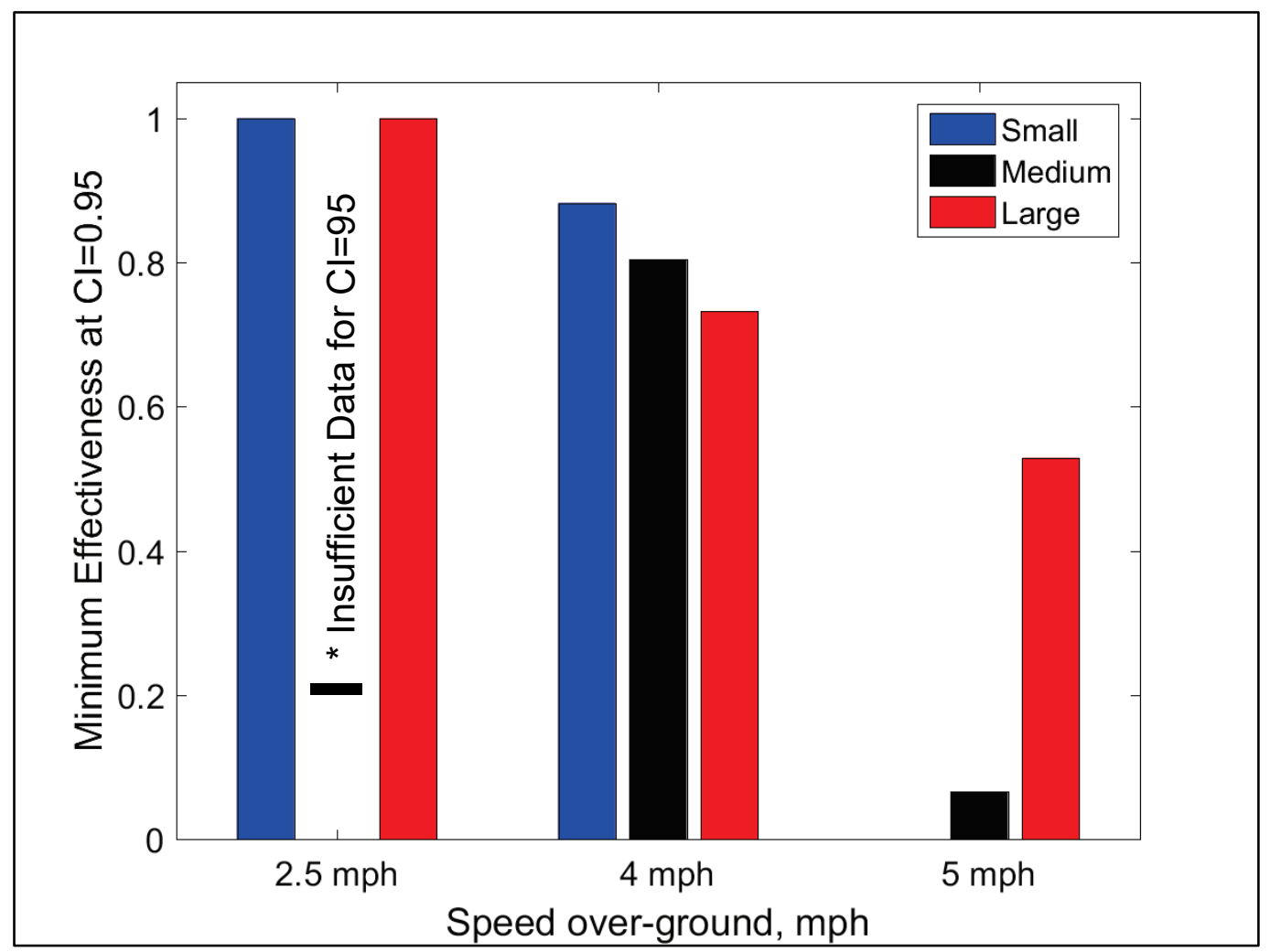

Table 6-9. Results of Level 4 alternate barge configurations for jet flushing tests.

\begin{tabular}{|c|c|c|c|c|c|c|c|c|c|c|c|}
\hline \multirow[b]{2}{*}{ 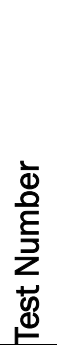 } & \multirow{2}{*}{ 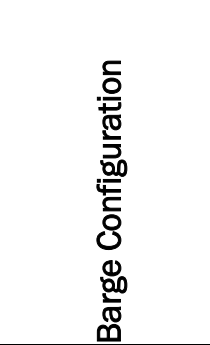 } & \multirow[b]{2}{*}{ 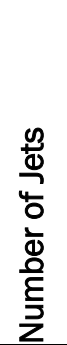 } & \multirow[b]{2}{*}{ 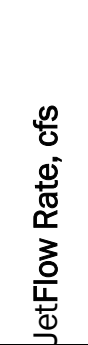 } & \multirow[b]{2}{*}{ 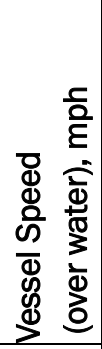 } & \multirow{2}{*}{ 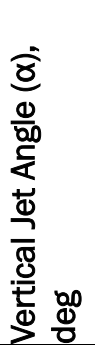 } & \multicolumn{5}{|c|}{ Flushing Proportion } & \multirow{2}{*}{ 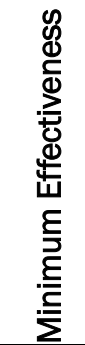 } \\
\hline & & & & & & R1 & R2 & R3 & R4 & R5 & \\
\hline 20 & Reverse rake & 18 & 430 & 2.5 & 67.5 & 0.95 & 0.95 & 1 & & & 0.88 \\
\hline 21 & Reverse rake & 18 & 430 & 5 & 67.5 & 1 & 1 & 1 & & & 1 \\
\hline 22 & Rake-rake & 18 & 430 & 2.5 & 67.5 & 1 & 0.95 & 1 & & & 0.89 \\
\hline 23 & Rake-rake & 18 & 430 & 5 & 67.5 & 1 & 0.95 & 1 & & & 0.89 \\
\hline
\end{tabular}

Using all the data for the Configurations 9, 11, 12, and 13, the proportion flushed (Figure 6-13) and minimum flushing effectiveness (Figure 6-14) vs. the vessel's speed over ground can be calculated. Both Figure 6-13 and Figure 6-14 clearly demonstrate that Configuration 9 did not perform as well as the other configurations as the vessel speed over ground increased. Configuration 9 is composed of entirely 12 in. prototype jets. Figure 6-14 shows that Configuration 11 composed of 8 in. prototype jets 
performed the best at high over-ground vessel speeds though this trend is not as discernable from Figure 6-13. Additionally, Figure 6-14 shows the dependence on the effectiveness of flushing jets with increasing overground vessel speed. The increase in over-ground vessel speed reduces the exposure to the flushing jets and their effectiveness. This problem can be overcome with an increase in flushing jet array length and pumping capacity.

Figure 6-13. Proportion flushed for all test tests vs. over-ground speed.

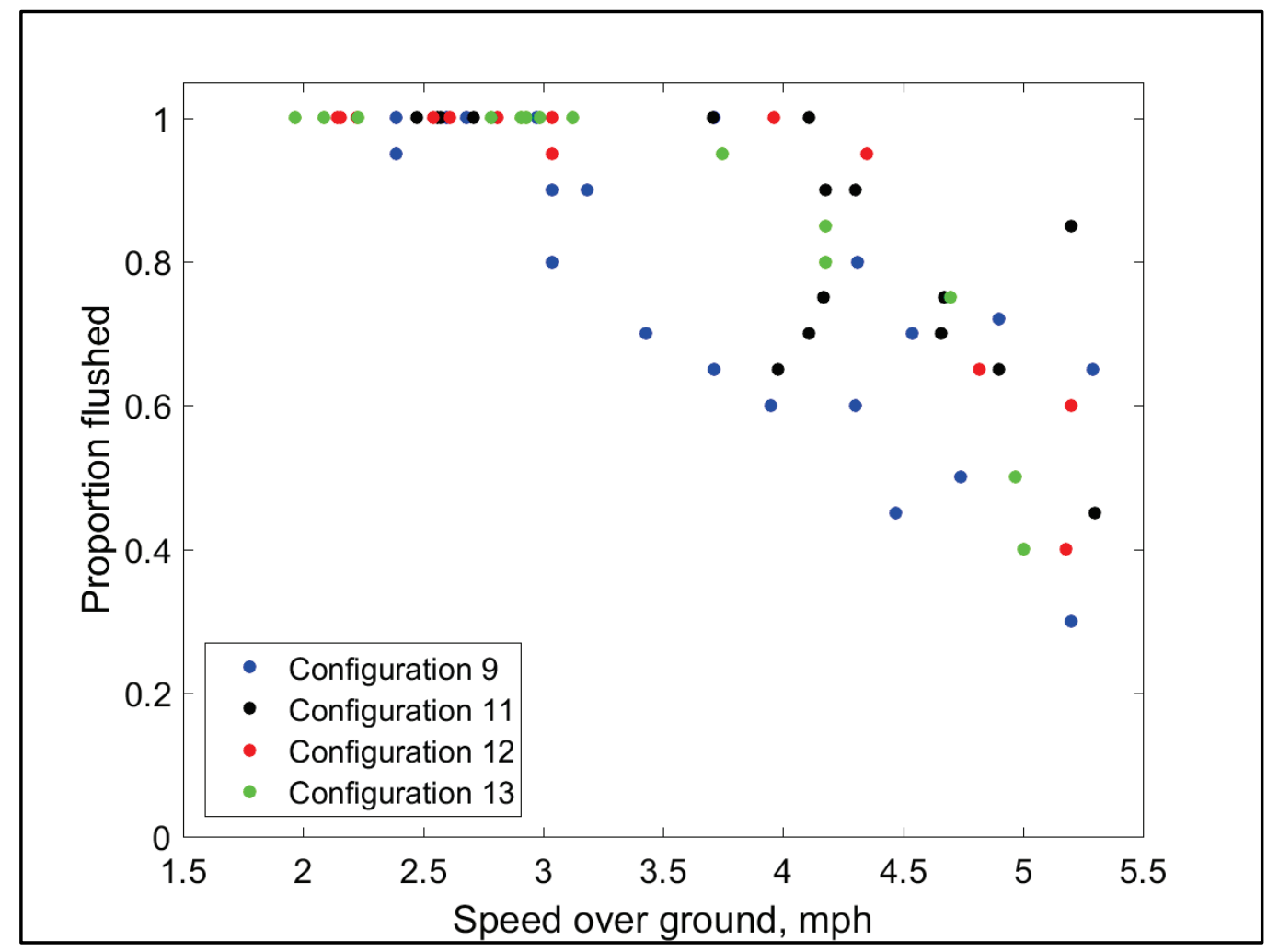


Figure 6-14. Minimum flushing effectiveness vs. over-ground speed.

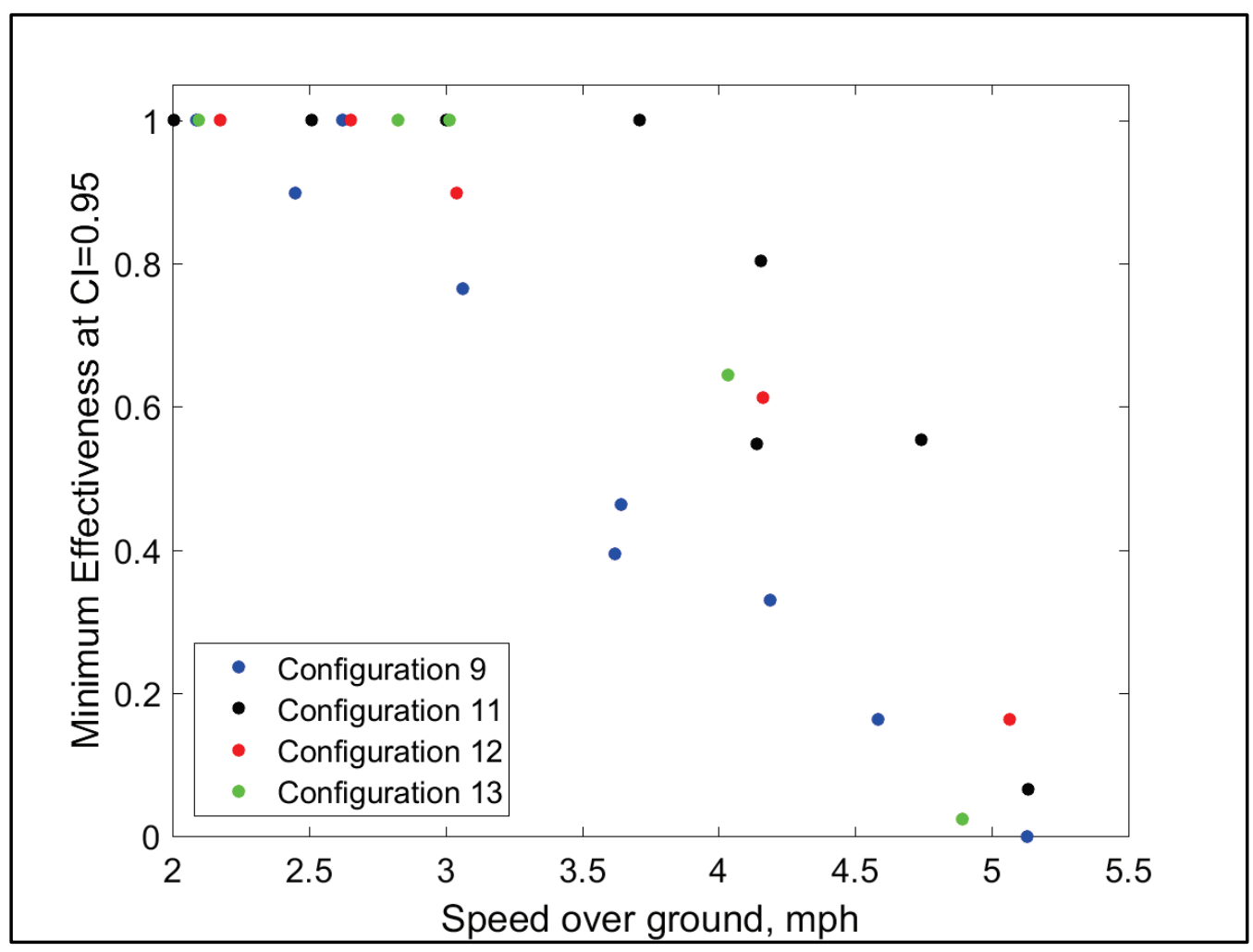

\subsection{Conclusions}

These results show that there are many different methods to reduce the risk of fish being entrained and transported with the barge. While all the maneuvers tested did not provide a high percentage of fish removal, the hard reverse did remove $100 \%$ of the fish every time. The flushing of fish from the vessel's path and around the barge by jets proved to be highly dependent on the jet configuration and jet flow rate. Vessel speed is shown to be an important parameter in determining how effective flushing jets can be. The tow's centerline moved directly over the jet array's centerline for every jet flushing test. Any deviation from this is expected to further reduce the effectiveness. Additionally, all the tests here used fish that are not actively swimming. Fish swim speed may be an important parameter in any final designs. Also, the safety aspects of surface impinging or flushing jets must be considered, as changes in vessel maneuverability associated with free surface deformations were encountered. 


\section{Discussion}

\subsection{Return current}

Return current measurements in the model compare well with those from the prototype, which indicates that the results presented in this report agree with the conditions at the electric barriers. As such, the return current characteristics measured in the physical model can be used to help explain the hydrodynamic conditions due to vessel passage at the electric barriers. Current measurements acquired at different lateral distances from the side of the canal (4.1, 9.0, and $13.9 \mathrm{ft}$ ) show the same increases in magnitude and duration, with little difference among the three locations. This similarity indicates the return current is fairly uniform across the width of the canal and does not decay appreciably near the channel sides, which is in agreement with the theory for confined channels (Maynord 1996).

\subsubsection{Spatial distribution of the return current}

Boundary layer effects will reduce the velocity adjacent to the channel walls. However, because the boundary layer is developing as the tow passes, the region of low velocity is confined near the wall. Once the tow passes, the return current vanishes and the wall boundary layer is no longer affected by the momentum flux created by the return current. This behavior limits the maximum boundary layer growth, and the associated region of low flow near the wall, due to the return current. Based on these results, incapacitated fish will experience a quasi-uniform return current as close as $4 \mathrm{ft}$ from the side of the canal with little boundary layer influence.

Laboratory tests to further explain the return current distribution include measurements at two depths ( $4.1 \mathrm{ft}$ and $13.1 \mathrm{ft}$ ) below the water surface and two vessel speeds ( 2.4 and $3.5 \mathrm{mph}$ ). Flow velocities at the two depths vary somewhat as a function of distance from the wall, but several of the tests produce overlapping error bounds (Figure 5-3). The return current for the higher vessel speed of $3.5 \mathrm{mph}$ is statistically higher at both depths. The variability between individual tests may be due to residual currents generated in the confined flume, small differences in vessel track, or propeller jet. Long waves can develop in the $500 \mathrm{ft}$ flume, which may produce weak flows through the test section that can generate currents in both directions depending upon the phasing of the wave during the test. However, a 20 min wait period between successive tests is implemented 
allows any spurious motions to be sufficiently damped. The tow driver took every reasonable measure to travel down the centerline of the flume, but small perturbations between tests did exist. Also, the velocity probes would have been damaged even with a glancing blow from the tow, which could have caused the tow driver to favor the non-instrumented portion of the flume. Last, the model tow can easily turn the propeller jet toward the velocimeters with the rudders. Since this study is not measuring the effect of the propeller jet, data collected where its occurrence was measurable were not considered. The propeller jet produces significant northbound velocities for southbound tows. Further evaluation of the potential impacts and migration strategies pertaining to propeller-jet entrainment would be needed to draw any conclusions.

While no direct visible sign was found to explain the variability, the data provide a measure of the return current variability and indicate that, while the differences between individual tests is statistically significant, the ensemble averages are representative of the return currents generated by tows. Fish in the canal during vessel passage will experience a fairly uniform return current adjacent to the barge from the surface to a depth of $13.1 \mathrm{ft}$.

\subsubsection{Effect of the tow configuration}

The magnitude of the return current is a function of vessel blockage ratio, which is the cross-sectional area of the barge divided by the cross-sectional area of the channel. Wider and deeper draft barges produce a greater blockage ratio and higher return current. The results show that the return current increases as a function of blockage ratio, with the 2-wide by 3-long barge configuration producing an average displacement distance nearly six times longer than the 1-wide by 1-long barge configuration (Figure 5-7). Longer and wider barge trains may expose stunned fish to higher velocities, which would increase the transport distance.

Overall, barge configuration can have a significant effect on return current variability and associated transport distance. The longer barge train increases the return current exposure time, allowing fish to be transported greater distances. A wider barge configuration increases the magnitude of the return current, which would increase the fish transport distance. Return current is lessened with a 1-wide by 1-long barge configuration and maximized by a 2-wide by 3 -long barge configuration. There is a steady increase in return current and distance traveled with increases in the number of barges. 
AIS records indicate that barge trains with five or six barges are common (Figure 2-2). The velocity distribution for loaded barges shows a peak at $3.75 \mathrm{mph}$, averaging 50 southbound tows per year. The total number of annual barge passages for one-to-two or three-to-four loaded barges is much greater. Thus, both the barge configuration data and the physical model measurements indicate that barge traffic in CSSC is capable of producing return currents that can transport passive particles across the width of the electric barriers with a single passage.

\subsubsection{Measures to mitigate the return current}

The return current for southbound tows represents a possible mechanism to transport stunned fish across the barrier. The physical model results indicate that the 2-wide by 2 -long and 2-wide by 3 -long barge configurations can transport passive particles distances greater than the width of the barriers. The tow velocities for these configurations vary between $0.4 \mathrm{ft} / \mathrm{s}$ and $0.9 \mathrm{ft} / \mathrm{s}$, which are typical of loaded barges in the CSSC. The natural flow in the channel is from north to south, which is opposite of the return current generated by southbound tows. The median cross-sectionally averaged flow velocity in the channel is $0.55 \mathrm{ft} / \mathrm{s}$, which is sufficient to overcome the return current for smaller barge trains. Considering that the results indicate that the smaller barge trains transport fish for distances that are less than the width of the barriers, combined with an opposing discharge that would further reduce fish transport, the return current for smaller barge trains (1-wide by 2-long; 1-wide by 1-long) is probably less of a concern, and fish would likely not cross the full width of the barrier. However, a loaded 2-wide by 3-long configuration can produce return currents of $0.8 \mathrm{ft} / \mathrm{s}$, so the discharge under average flow conditions cannot overcome all possible return currents generated by larger barge trains. During periods of low flow, the discharge will not be able to overcome the return current, and the likelihood of fish being carried across the barrier increases.

One alternative to mitigate the effects of the return current is to maintain a minimum discharge in the canal. This approach would require either modifying the operational procedures of the existing control structures that regulate flow in the CSSC or locally controlling the discharge near the barriers. Modifying the operational procedures of control structures in the CSSC would require coordination with the Metropolitan Water Reclamation District of Greater Chicago, which regulates flow discharge in the CAWS. Coordination efforts would include determining the minimum 
recommended discharge and developing operational protocols to ensure that when barge trains pass the barriers, the discharge produces sufficient flow velocities to counteract the return current. Locally controlling the discharge near the barriers would require developing a flow system to maintain a minimum discharge near the electric barriers that could counter the return current.

Another alternative would be to regulate barge traffic during periods of low flow. Southbound tows would not be permitted to cross the barriers under prescribed low discharge conditions. This option would require coordination with the towing industry, USCG, and other stakeholders to ensure safe navigation.

\subsubsection{Modeling return current at the barriers}

Given the significant correlation between the physical model and prototype, a model of the return current could be constructed given tow speed, barge draft, and barge configuration. The model could be integrated into a monitoring system of tow traffic across the barriers to produce estimates of return current and distance traveled. The average channel velocity could also be subtracted from the return current to predict the net velocity in the channel. The net velocity could then be used to obtain estimates of the distance a particle (stunned fish) could be transported per barge passage and compared to the width of the barriers. Assessing the likelihood that an approaching southbound barge could generate a return current sufficient to transport fish across the barrier could then be possible, measures could be initiated to reduce the tow speed such that the maximum transport distance is less than the barrier width.

\subsection{Entrainment - vessel maneuvers}

Bryant et al. (2016) showed that entrainment due to vessel-generated currents could transport model fish across the electric barriers. Because entrainment is a function of the vessel characteristics that generate the flows responsible for entrapping fish (draft, barge configuration, geometry, speed, direction, etc.), some vessel maneuvers may act to counter the trapping tendency and produce currents that repel or dislodge fish. An alternative was conceived to use vessel maneuvers to try to remove or dislodge entrained fish for northbound tows. In all maneuver alternatives, the configuration included a leading box-end barge followed by a rake barge. This represents a worse-case scenario, as the previous 
entrainment study showed that this configuration retained the greatest number of model fish and transported them the largest distances.

\subsubsection{Lateral vessel movement}

The first alternative involved a swing maneuver. The starboard stern of the towboat is moored to the channel wall, and the port engine is set in reverse until the head of the tow reached the center of the channel. Once the head of the tow reached the center of the channel, the stern line is released, and the stern is swung out to meet the head. The rationale behind this alternative is to generate a cross-flow in the recess between barges thereby transporting the model fish laterally as the tow rotates to starboard.

A variation to this alternative that did not include mooring the vessel was also tested. Instead, the tow moved uniformly away from the wall as opposed to pivoting at one end. Five tests were conducted for both alternatives, and none were effective at removing fish. The fish remained in the recess while the barges separated from the wall. Once the tow was in the channel, the fish remained entrained. Forward motion kept the model fish in the recess, as had been demonstrated in the previous entrainment study.

\subsubsection{Vessel reverse}

A second alternative, which involved bringing the tow to rest and then reversing engines, was also evaluated. The reversing tow generated lateral currents within the recess between barges that flushed the fish outward. All tests were $100 \%$ successful at removing fish. The mechanism was likely effective because the flow reversal beneath the tow combined with the barge configuration produced favorable pressure differences that transported the model fish laterally. In the barge's frame of reference, the flow moved along the bottom of the barge until it encountered the trailing rake junction. The flow moved slightly upward along the oblique rake and then impinged on the vertical box end barge. The impinging flow increased the dynamic pressure causing upwelling along the box end barge. Although not directly measured, this upwelling would lead to a slight increase in surface elevation along the box end barge similar to, but much lower in magnitude than, the bow wave generated along a leading edge barge. At the lateral ends of the barge junction, the flow was exposed to the open channel, and the moving barge produced a drawdown. The prototype-scale barge tests in the CSSC showed an elevation difference between the water surface just inside the edge of the junction and the free 
surface outside of the junction. This difference produced a lateral pressure gradient causing the water in the recess to flow outward. By symmetry, both ends of the barge junction experience the same pressure drop so that model fish are transported laterally until they are outside the recess.

\subsection{Entrainment - flushing}

The jet flushing alternatives comprised the majority of the physical model testing. The jet concept was a new approach, so developing a number of different design configurations, and evaluating each of them to determine their potential effectiveness was necessary. The flushing alternatives were designed to remove fish from the recesses before crossing the barrier and applied to northbound tows. Fish that could become entrained in southbound tows would be carried toward the south side of the barriers and away from Lake Michigan. The jet manifold was placed in the center of the channel, and the tow was piloted to remain close to the centerline. The jet struck the bottom of the barge train at its center point such that the flushing action moved fish laterally and away from the barge.

\subsubsection{Jet placement - vertical jets}

One of the first issues was developing a jet design that effectively flushed fish. One early design included a jet manifold aligned along the channel axis with vertical jets. The vertical jet was not $100 \%$ effective at clearing fish. The tests showed that the vertical jets generated vigorous upwelling at the surface, which did displace model fish from the center of the recess. As the fish were transported laterally, the jet strength diminished and eddies formed, causing the model fish to move in quasi-circular motions but not necessarily toward either end of the barge junction. Since that the standard of success was to clear all model fish, this design was not considered effective. One possible modification that may have cleared more model fish would be to increase the number of jets and the length of the manifold. This modification would increase the exposure time and allow the turbulent motions to eventually clear the model fish. However, other alternatives were more effective using the same manifold design.

\subsubsection{Jet placement - forward-angled jets, low vessel speed}

A number of tests with both 45 and 67.5 deg jets were conducted. The $45 \mathrm{deg}$ angle was less effective than the $67.5 \mathrm{deg}$ angle and is not discussed. The jets angled at $67.5 \mathrm{deg}$ off the vertical pointed forward (in 
the direction of barge travel) in an alternating increasing angle (Figure 7-1). The 67.5 deg angle permitted the jet to impinge obliquely, producing a more direct flow against the box end barge as the tow passed overhead. This configuration was inspired by the successful hard reverse maneuver, which effectively produced a flow impinging on the box end of the barge. At the slower vessel speeds $(2.5 \mathrm{mph}$ ) this configuration was $100 \%$ effective with the 12 in. nozzle and $80 \%$ effective with the 8 in. nozzle. The larger diameter reduced flow velocity but increased the width of the momentum flux, thereby moving more mass per nozzle. The angled jet promoted lateral transport, and all the model fish were cleared of the recess for all tests.

Figure 7-1. Manifold configuration for successful jet tests. Barge direction is from the top of the photograph.

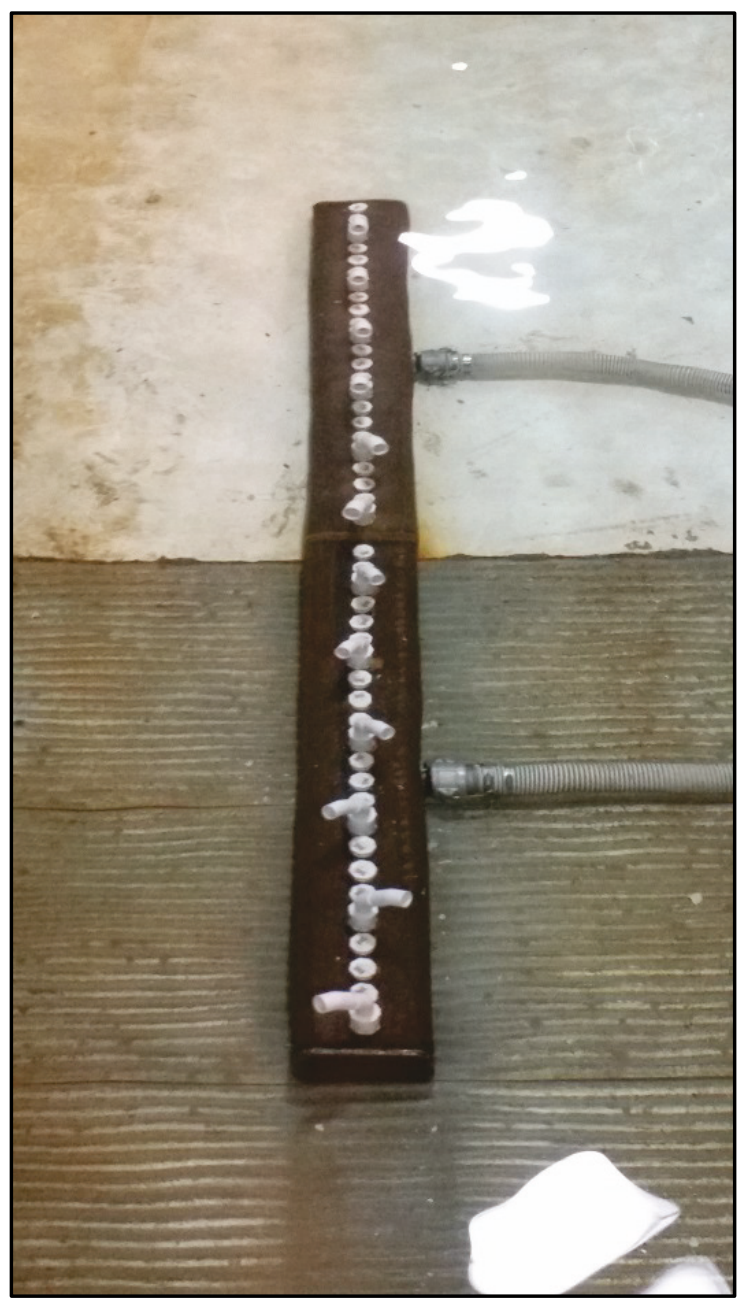




\subsubsection{Jet placement - forward-angled jets, high vessel speed}

AIS data indicated that loaded barges traverse the CSSC at speeds between 3 and $5 \mathrm{mph}$ (Figure 2-5). The smaller barge configurations (1-wide by 2-long) tend to travel near the higher end of the limit, and the larger barge configurations (2-wide by 3-long) tend to travel near the lower end. Flushing tests with vessel speeds of 4 and $5 \mathrm{mph}$ were conducted for the angled jets and two different discharges (330 and $430 \mathrm{cfs}$ ). Arrays of either 12 or 18 jets were used in the tests (see Tables 6.5 and 6.6).

In all cases, the tests failed to achieve a 100\% success rate. For the lower discharge tests (330 cfs), the minimum effectiveness was less than $22 \%$. For the higher discharge tests (430 cfs), the minimum effectiveness was less than $80 \%$. Since the standard for success was to remove all model fish, the higher vessel speed tests showed that this jet placement was not a viable alternative. The main contributing factor to the lower minimum effectiveness was the increased vessel speed. The increased speed reduced the exposure time to the jets, so the model fish were not subjected to the flushing action long enough to be removed from the barge junction recess.

Increasing the length of the manifold and the number of jets would increase exposure time, possibly allowing the flushing action to remove all the model fish. Increasing the manifold length was not tested because the primary objective of this proof-of-concept study was to determine if flushing might be a viable mitigation option by exploring possible jet configurations that may work under reasonable conditions at the barriers. The results suggested that limiting maximum vessel speeds to $2.5 \mathrm{mph}$ across the jet array could be an effective measure. However, further testing at prototype-scale would be necessary to fully establish the operational parameters needed to achieve the required flushing effectiveness.

\subsubsection{Jet placement - canal flow}

Additional flushing tests were conducted for the more realistic case of flow in the canal. Based on the discharge measurements (Figure 2-2), the dominant flow is from north to south with a median current of $0.55 \mathrm{ft} / \mathrm{s}$. Tests with 2,500 cfs and 6,000 cfs, which represent the 5oth percentile and 95th percentile of flows measured in the prototype channel, respectively, were conducted with the angled jets and a vessel speed of $5 \mathrm{mph}$. The direction of the canal flow was opposite to the angled jets and could reduce the flushing effectiveness. The tests indicated that increased 
discharge led to higher flushing efficiency, but the higher discharge did not achieve a minimal effectiveness of $100 \%$. This result indicated that the effect of discharge did not substantially disrupt the jet velocities as higher discharge did not decrease the effectiveness compared to the tests without flow in the canal. The greater effectiveness is likely due to the decrease in vessel speed over the jet array. During the tests without flow in the channel, the vessel speed was the same relative to the jet array and the still water. Introducing flow into the channel did not change vessel speed relative to the water, but it did reduce the speed relative to the channel bottom and the jet array. This increased the exposure time, as the tow passed over the array at a slower speed compared to the tests without flow in the channel. This allowed the jet stream to maintain longer contact with the barge junction and flush more model fish.

\subsubsection{Navigation safety}

The jets created a concentrated flow field that could affect navigation. The tow in the physical model was remotely controlled and traveled under its own power. The same operator piloted the tow for all tests and was able to evaluate vessel handling as it traversed the jets. The tests were performed with the vessel moving at constant speed along a straight path in the center of the channel. The jet manifold was placed in the center of the channel and aligned parallel to the channel walls. This testing pattern was designed to maximize symmetry in the channel, which was considered the safest approach as the tow remained near the centerline and away from the channel walls. This gave the operator maneuvering room in case the tow was driven off course by the jets. Minor steering adjustments were sometimes necessary when traversing the array, but the operator was able to maintain full control of the tow. To ensure safe navigation in the CSSC, the potential effects of the jet-induced velocities interfering with navigation should be included in any testing program at the prototype scale.

\subsubsection{Jet manifold design alternatives that demonstrated lower levels of success}

Other manifold configurations were initially tested but dismissed as potential alternatives because of the low numbers of model fish that were flushed. These configurations are briefly mentioned here to explain the hydrodynamics of vessel-jet interactions and to serve as a guide and provide lessons learned to inform the design and further testing of an operational system. 
Lateral array across the channel. In this configuration, the manifold was rotated 90 deg from the channel's longitudinal axis so the nozzle array extended perpendicular to the path of the tow. Both vertical and angled jets (with jet angle pointing parallel to the channel axis) were tested. As the barge passed over the array, the flushing action was applied to the full width of the junction. By flushing the width of the recess simultaneously, the wide jet array would rapidly transport the fish from the barge junction. This configuration was unsuccessful because the reduced exposure time did not allow the persistent flow needed to remove all the model fish to be generated. If the tows were brought to rest over the array, this flushing mechanism may be more effective.

Oblique array across the channel. In this configuration, the manifold was rotated $45 \mathrm{deg}$ from the channel axis, so the nozzle array extended obliquely to the path of the tow. The forward motion of the barge passing over the array caused each successive jet to focus on a different section of the barge junction. The leading nozzle was located off-center of the barge, so the overall effect produced a sweeping motion that forced fluid laterally across the recess as opposed to the longitudinal manifold. This lateral flow produced a divergent flow that forced fluid in both directions. Both angled and vertical jets were tested and failed to flush all model fish. This configuration was a hybrid between the longitudinal array, which was successful at low speeds, and the lateral array, which was ineffectual due to the low exposure time. As such, increasing the length of the manifold could be more effective, which would increase the exposure time, and allow the jets to act long enough to maintain a persistent flow field and remove more fish.

\subsection{Other potential alternatives}

In addition to the scheduled tests, the project team discussed potential alternatives that were not tested but may be viable mitigation measures for further consideration in the future. Unlike the return current or jet tests, these alternatives require modifications to the canal or vessel or require that the vessel conduct maneuvers atypical of normal operations. The following recommendations have not been tested, so there are no data to support the requirements to prescreen these alternatives or to demonstrate their potential effectiveness. The feasibility of these approaches requires further development before recommending testing. 


\subsubsection{Return current blockage system}

The study showed that the return current for the larger barge configurations could transport fish across the width of the barrier. One alternative included a blockage system that would prevent fish from being transported by the return current. The system design includes permeable mesh gates that extend laterally from the walls to a point about one-third of the distance across the channel (Figure 7-2). This mesh would allow water to flow in the canal but prevent the return current from transporting fish. The gates would extend from the surface to a depth where the electric field strength is sufficient to kill the fish. Below this point, the fish would experience a return current, but the higher voltage would kill them before they could cross the barrier. The exact depth is unknown and would need to be investigated as would the design of the gate. The flexible gate would open to accommodate the width of the barge train as the tow passes the barrier and then return to its original position once the tow is clear. The gate would operate automatically or be forced open by the moving barge.

Figure 7-2. Schematic illustrating return current gate blocking system. Water flows through the mesh gates but prevents fish from moving with the return current.

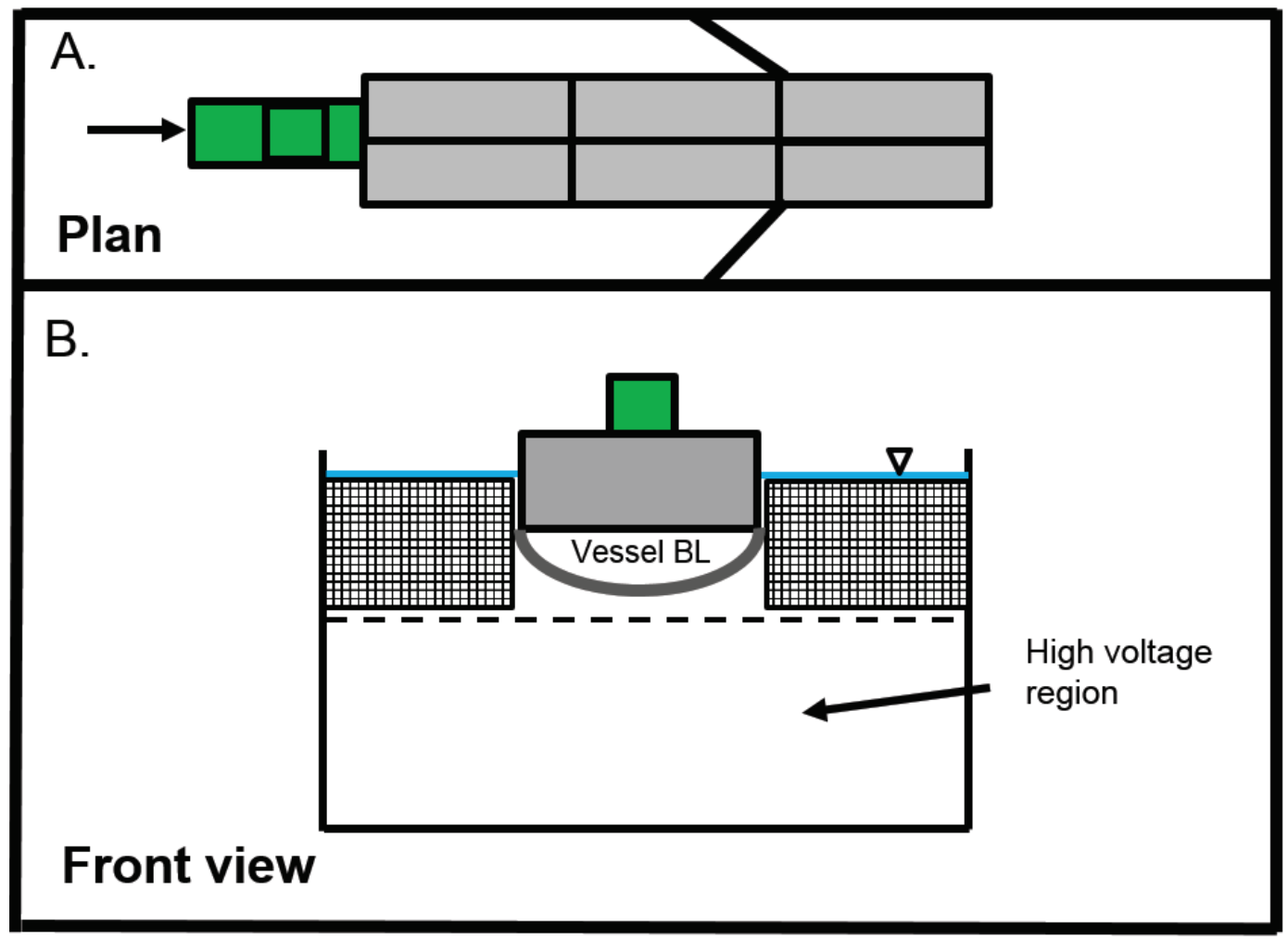


Another alternative is to install bumpers on the channel walls (Figure 7-3). This is similar in concept to the mesh gate except the bumpers are solid and would block the return current near the surface, forcing the bulk of the flow beneath the draft of the vessel. Fish below the bumpers would be subjected to the return current but would be killed by the higher voltage. The bumper could be constructed of a flexible polymer to allow the passing barges to graze the sides without damage.

Figure 7-3. Schematic illustrating bumper system. Similar in concept to the gate in that fish are deflected downward into the high-voltage region.

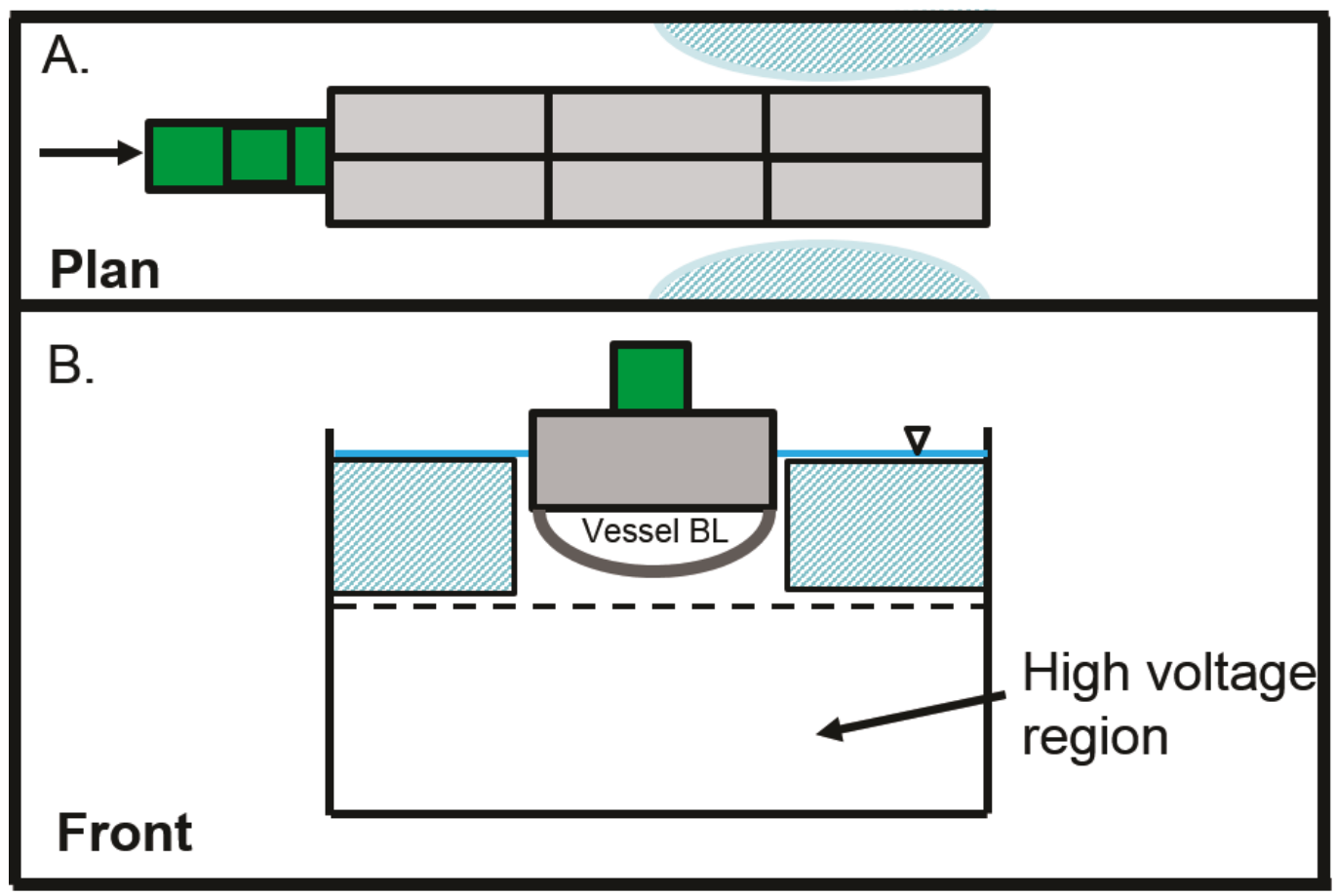

\subsubsection{Bladders at barge junction recesses}

This study showed that the flow formed between barge junction recesses is a primary mechanism to entrain and retain fish. As the flow moves along the bottom of the barge and encounters a raked junction, the expansion induces an upward current that forms an eddy in the recess. Observations from the first barrier study (Bryant et. al 2016) show that fish advected upwards into the void space and were then carried along with the barge. One possible mechanism to mitigate the effect of barge junction entrainment is to effectively plug or block the recess. Inflatable bladders could be installed in the rake junction, thereby prohibiting eddy formation and associated entrainment currents (Figure 7-4). 
Figure 7-4. Schematic illustrating possible alternatives for reducing fish entrainment. (a) fender, (b) bladder and/or skirt.

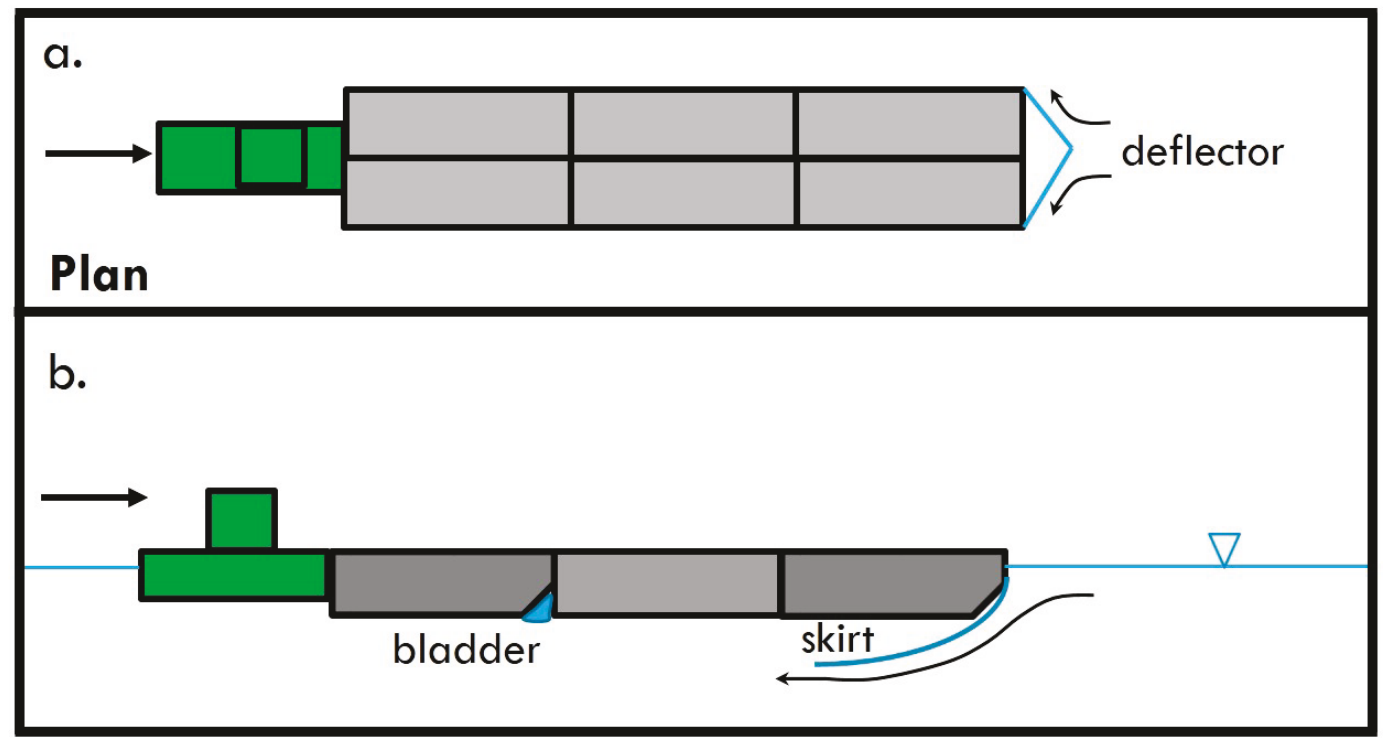

The bladder could be constructed from polymer material and stored on the barge or towboat when operating away from the barriers. When preparing to cross the barrier, the bladder could be installed between the barges and filled with water via an onboard pump. Because the filled bladder would have nearly the same density as the water, it would not produce any added mass to the tow. The flexible bladder would conform to the shape of the junction, increasing its effectiveness by reducing potential entrainment zones. The bladder would be portable and would require a commercial pumping system. The towboat crew could be trained to install the system as necessary.

\subsubsection{Fender system}

Removing fish from the vessel path before entering the barrier is another option to reduce the likelihood of entrainment. If the fish are forced from the barges path, they cannot move along the bottom of the hull and become trapped in the barge junction recesses or caught in the residual currents generated by the towboat. This approach is similar in concept to thruster barges mounted at the front of a barge train to aid in maneuvering. A fender with a protruding nose cone mounted on the leading barge would deflect fish latterly as the barge passes the barrier (Figure 7-4). The fender allows the tow to effectively plow through a school of fish and force them to either side of the barge, which is similar to a how pilot (e.g., cow catcher) on a locomotive works. The fender could be installed on the leading barge, or a modified system for 2-wide barge 
trains, before crossing the barrier perhaps at one of the bounding locks. Once the tow is no longer in the barrier region, it could be removed and stored locally or with the towboat. A small suite of barges could be outfitted with a fender for tows that routinely operate near the barriers. This would avoid the need to install the fender before each barrier crossing.

This alternative will deflect fish in the direct path of the barge but is ineffective for fish on either side or below the draft of the barge. Fish in these regions are much less likely to be entrained by lateral or upward flows that could transport fish closer to the tow's zone of influence. The previous study (Bryant et al. 2016) showed that fish at the bottom or on the side of the barge are influenced by the return current, which for upbound tows carries fish in the direction opposite of the electric barrier.

\subsubsection{Skirts}

Another option is to deflect fish deep beneath the barge, such that the barge passes over the fish before they could rise to the surface and become entrained. This method follows a similar principle as the fender system in that the fish are removed from the direct influence of the vessel-generated currents. This alternative consists of a skirt constructed of flexible and permeable material that is draped over the front of the leading barge forcing fish deep below the tow (Figure 7-4). The permeable skirt would reduce flow resistance and use the vessel-generated currents to provide a smooth curved surface to limit the chance of fish becoming entangled. This alternative would require the skirt to be installed on the leading barge and then deployed before reaching the barriers. The system could be stored on a spool and then unrolled off the front of the barge when necessary. Multiple skirts could be installed on interior barges to increase their effectiveness.

The skirt would force fish below the barge into the region of higher voltage, thereby increasing the effectiveness of the barriers. Like the fender system, the skirt does not clear fish beyond the width of the tow. However, fish outside the tow's influence are less likely to be entrained during transit. 


\subsubsection{Dock-based jets}

This alternative involves temporarily docking the tow and then dislodging entrained fish with a jet system (Figure 7-5). This method is similar to the docking maneuver but has the added benefit of flushing jets. The tow would maneuver to a docking station in the canal, and once docked, a suite of sidewall and perhaps bottom-mounted jets would sweep entrained fish from the tow. Once the fish are cleared, the tow would resume normal operations and traverse the barriers.

Figure 7-5. Schematic illustrating docking maneuvers and jet wash to remove entrained fish.

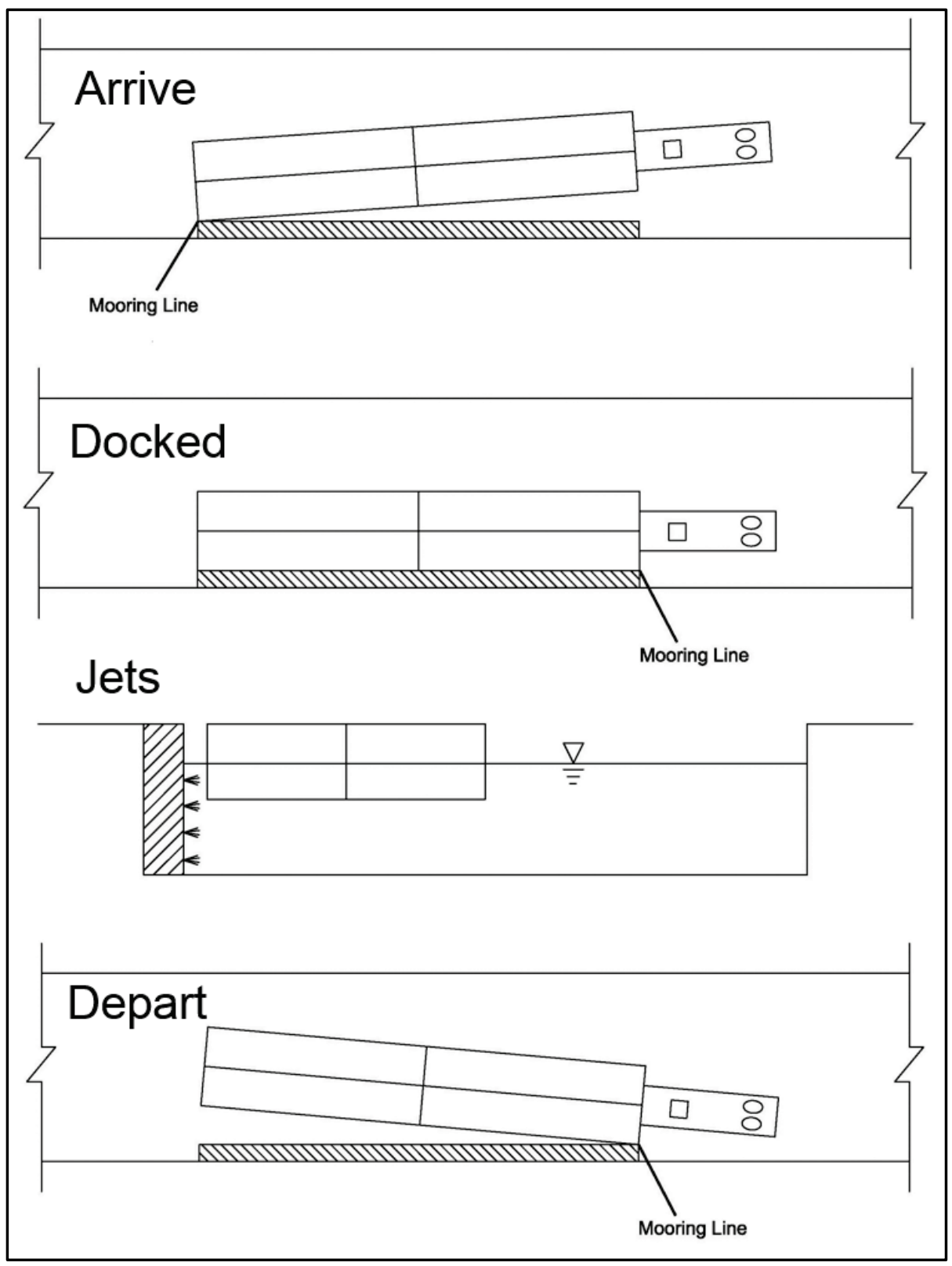




\section{Summary and Conclusions}

A previous physical model study (Bryant et al. 2016) demonstrated that commercial tows operating in confined channels produce residual currents and forces that entrain and transport immobile fish. At the request of the U.S. Army Engineer District, Chicago, the U.S. Army Engineer Research and Development Center (ERDC), Coastal and Hydraulics Laboratory, was tasked to investigate mitigation measures to remove entrained fish from the recesses between barges and to reduce the effect of the return current in transporting fish across the barriers. A series of tests were carried out in a 1:16.7 scale physical model that represented the Chicago Sanitary and Ship Canal (CSSC) near the electric barriers. The tests included measurements of the return current to determine particle displacement and installation of an array of upward looking jets to displace entrained model fish from the recess between barge junction recesses.

\subsection{Return current}

The return current in the physical model was validated with measurements collected in the CSSC. The shape and magnitude of the return velocity was well correlated with the prototype. As such, the return current characteristics measured in the physical model were used to help explain the hydrodynamic conditions due to vessel passage at the electric barriers.

The results showed that barge configuration had a significant effect on return current variability and associated transport distance. The longer barge train increased the return current exposure time, allowing fish to be transported greater distances. The wider barge configurations increased the blockage ratio, which increased the magnitude of the return current. For the larger barge configurations (2-wide by 3-long), the physical model measurements indicate that passive particles could be transported the width of the electric barriers during a single tow passage. During periods of higher discharge, the flow in the canal can overcome the return current reducing fish transport distance. Potential mitigation measures include eliminating or reducing the return current by creating a flow in the canal that was either locally produced at the barrier or by controlling the canal discharge itself. Restricting southbound tow passage during periods of low discharge may also be a potentially effective alternative. 


\subsection{Flushing jet}

The flushing alternatives were designed to remove fish before crossing the barrier and applied to northbound tows. The design included a manifold placed on the bottom of the canal with a series of upward facing jets that impinged on the bottom of the barge during passage. A number of trial tests with different manifold and jet configurations were conducted to determine which method removed the greatest number of fish. The results indicated that a jet oriented at 67.5 degrees from the horizontal with a 12-inch nozzle had a minimum effectiveness of $100 \%$ for a 2-wide by $3^{-}$ long barge configuration traveling at 2.5 miles per hour (mph). At higher vessel speeds ( $4-5 \mathrm{mph}$ ), the jets were unable to remove all fish. The main contributing factor to the lower minimum effectiveness was the increased vessel speed, which reduced the exposure time so the model fish were not subjected to the flushing action long enough to be removed from the barge junction recess. Increasing the length of the manifold and the number of jets would increase exposure time, potentially allowing the flushing action to remove all the model fish. As another alternative, limiting maximum vessel speeds across the jet array could be an effective measure.

\subsection{Other considerations and recommendations}

This report also outlines potential mitigation measures that show promise but were not tested. For southbound tows these included the following: (1) installing a permeable gate in the canal that would prevent fish from being carried by the return current and (2) installing a bumper system that would block fish or force them toward the bottom of the canal where the electric field strength is greatest. For northbound tows these included the following: (1) modifying the leading barge with a skirt to deflect fish downward and away from the barge influence, (2) adding a deflector to the leading barge to deflect fish laterally from the path of the vessel, (3) installing a bladder between barge junction recesses to physically block fish entrainment, and (4) docking the barge temporarily and flushing fish with a side-mounted jet system.

Given the success of the flushing jets shown in the physical model, future work could include a prototype-scale study to test the feasibility of this approach. A prototype-scale array could be installed in the CSSC, and a similar set of tests as performed in the laboratory could be replicated. The engineering requirements for a prototype-scale test would need to be 
developed, but the idea is feasible in light of previous field work to examine barge-induced fish transport in the CSSC.

The physical model studies thus far have assumed that the fish are immobilized without considering behavioral characteristics. The assumption is that once fish are stunned, then they are transported passively with the barge or in the return current. As an intermediate approach, a laboratory investigation involving live fish and upward facing jets could be conducted. These sorts of experiments would provide greater control of the testing procedures with the benefit of evaluating flushing effectiveness given the unknown consequences of fish behavior. The disruptive effect of the jet flow alone could be sufficient to drive fish away from the tow's region of influence. This possibility means that a wider array with weaker jets may suffice to keep fish away from tows without the need to produce strong enough flows to remove fish. The testing procedures would need to be developed to determine the degree to which the laboratory results would translate to the prototype. However, several ongoing studies of Asian carp behavior at ERDC use laboratory scale facilities to infer behavioral characteristics at the prototype scale. The success of these behavioral studies could translate into an effective laboratory investigation of barge entrainment using actively swimming fish. 


\section{References}

Bec, J., L. Biferale, G. Boffetta, A. Celani, M. Cencini, A. Lanotte, S. Musacchio, and F. Toschi. 2006. "Acceleration Statistics of Heavy Particles in Turbulence." Journal of Fluid Mechanics 550:349-358. https://doi.org/10.1017/S002211200500844X.

Bryant, Duncan, B., Stephen T. Maynord, Howard E. Park, Lauren Coe, Jarrell Smith, and Richard Styles. 2016. Navigation Effects on Asian Carp Movement Past Electric Barrier, Chicago Sanitary and Ship Canal. ERDC/CHL TR-16-2. Vicksburg, MS: U.S. Army Engineer Research and Development Center. http://hdl.handle.net/11681/21560.

Maynord, S. T. 1996. Return Velocity and Drawdown in Navigable Waterways. Technical Report HL-96-7. Vicksburg, MS: U.S. Army Engineer Waterways Experiment Station. http://hdl.handle.net/11681/13178.

Maynord, S. T. 2000. Physical Forces near Commercial Tows: Interim Report for the Upper Mississippi River-Illinois Waterway System Navigation Study. ENV Report 19. Vicksburg, MS: U.S. Army Engineer Research and Development Center. http://acwc.sdp.sirsi.net/client/en_US/default/index.assetbox.assetactionicon.view/1052388 ? rm=ERDC+DIGITAL+R0\%7C\%7C\%7C1\%7C\%7C\%7C0\%7C\%7C\%7Ctrue.

Maynord, S. T., and S. K. Martin. 1997. Physical Forces Study, Kampsville, Illinois Waterway: Interim Report for the Upper Mississippi River-Illinois Waterway System Navigation Study. ENV Report 3. Vicksburg, MS: U.S. Army Engineer Waterways Experiment Station. http://acwc.sdp.sirsi.net/client/default/search/detailnonmodal/ent:\$002f\$002fSD_ILS\$002f 0\$002fSD ILS:87293/ada?rt=CKEY山CKEY山 Walse.

Maynord, S. T., and S. K. Martin. 1998. Physical Forces Study, Clarks Ferry, Mississippi River: Interim Report for the Upper Mississippi River-Illinois Waterway System Navigation Study. ENV Report 5. Vicksburg, MS: U.S. Army Engineer, Waterways Experiment Station.

http://acwc.sdp.sirsi.net/client/default/search/detailnonmodal/ent:\$002f\$002fSD_ILS\$002f 0\$002fSD_ILS:87292/ada?rt=CKEY|||CKEY|||false.

Permanent International Association of Navigation Congresses (PIANC). 1987. Guidelines for the Design and Construction of Flexible Revetments Incorporating Geotextiles for Inland Waterways. Report of Working Group 4, Permanent Technical Committee 1, Supplement to Bulletin No. 57. Brussels, Belgium: Permanent International Association of Navigation Congresses. http://www.pianc.org/edits/articleshop.php?id=1000041.

Schijf, J. B. 1949. "Protection of Embankments and Bed in Inland and Maritime Waters, and in Overflow or Weirs." In Proceedings, Seventeenth International Navigation Congress, Lisbon, Portugal. Section 1. Subject 2. 61-78. https://repository.tudelft.nl/islandora/object/uuid:e0a52ec1-b6d3-475d-b50c$80 \mathrm{e} 6 \mathrm{c} 6 \mathrm{e} 2 \mathrm{fe} 0 \mathrm{e}$ ?collection=research. 
Schlichting, H. 1968. Boundary Layer Theory. New York: McGraw-Hill.

https://repository.tudelft.nl/islandora/object/uuid:e0a52ec1-b6d3-475d-b50c-

80e6c6e2fe0e?collection=research. 


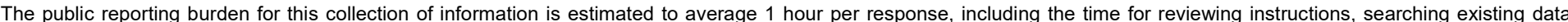

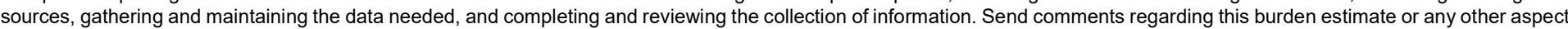

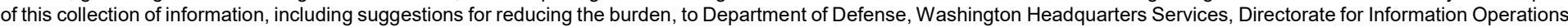

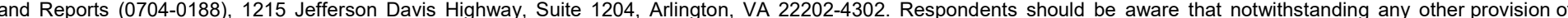
law, no person shall be subject to any penalty for failing to comply with a collection of information if it does not display a currently valid OMB control number.

PLEASE DO NOT RETURN YOUR FORM TO THE ABOVE ADDRESS.

\begin{tabular}{l|l|l}
\hline $\begin{array}{l}\text { 1. REPORT DATE } \\
\text { August } 2018\end{array}$ & $\begin{array}{l}\text { 2. REPORT TYPE } \\
\text { Final Report }\end{array}$ & 3. DATES COVERED (From - To) \\
\hline
\end{tabular}

\section{TITLE AND SUBTITLE}

Laboratory Study to Reduce the Transport of Asian Carp by Barges 5a. CONTRACT NUMBER

5b. GRANT NUMBER

5c. PROGRAM ELEMENT NUMBER

5d. PROJECT NUMBER

114532

5e. TASK NUMBER

5f. WORK UNIT NUMBER

8. PERFORMING ORGANIZATION REPORT NUMBER

ERDC/CHL TR-18-14

10. SPONSOR/MONITOR'S ACRONYM(S) USACE LRC

11. SPONSOR/MONITOR'S REPORT NUMBER(S)

\section{DISTRIBUTION/AVAILABILITY STATEMENT}

Approved for public release; distribution is unlimited.

\section{SUPPLEMENTARY NOTES}

\section{ABSTRACT}

Potential migration of Asian carp through the Illinois River, Des Plaines River, and Chicago Area Waterway System is a risk facing the Great Lakes. The U.S. Army Corps of Engineers (USACE) has installed electric barriers within the Chicago Sanitary and Ship Canal (CSSC) near Romeoville, IL, to deter fish from using the canal as a pathway to enter the Great Lakes. Commercial tows operating within the CSSC produce residual currents that can entrain and potentially transport fish across the barrier. The U.S. Army Engineer Research and Development Center was tasked by the USACE Chicago District to investigate mitigation measures to remove entrained fish from the recesses between barges and to reduce effects of return currents in transporting fish across the barriers. A 1:16.7 scale physical model with remote-controlled tow and barges was used to evaluate mitigation strategies. For southbound tows, mitigation measures include maintaining a minimum discharge in the canal to overcome the return current or reducing vessel speeds across the barriers. For northbound tows, mitigation measures include vessel maneuvers and upward facing jets to dislodge or remove fish during passage. These jets were effective at flushing fish for lower vessel speeds (2.5 miles per hour).

\section{SUBJECT TERMS}

Barges, Bighead carp-Migration, Introduced aquatic organisms, Nonindigenous pests, Silver carp-Migration, Waterways

16. SECURITY CLASSIFICATION OF:

\begin{tabular}{|l|c|c|c|} 
a. REPORT & b. ABSTRACT & c. THIS PAGE & ABSTRACT \\
Unclassified & Unclassified & Unclassified & SAR
\end{tabular}

18. NUMBER OF PAGES

106 19a. NAME OF RESPONSIBLE PERSON

Duncan. B. Bryant

19b. TELEPHONE NUMBER (Include area code) 601-634-3898 